\title{
Redshift space power spectrum beyond Einstein-de Sitter kernels
}

\author{
Alejandro Aviles, ${ }^{a, b}$ Georgios Valogiannis, ${ }^{c}$ Mario \\ A. Rodriguez-Meza, ${ }^{b}$ Jorge L. Cervantes-Cota, ${ }^{b}$ Baojiu Li, ${ }^{d}$ \\ Rachel Bean ${ }^{e}$
}

\author{
${ }^{a}$ Consejo Nacional de Ciencia y Tecnología, Av. Insurgentes Sur 1582, Colonia Crédito \\ Constructor, Del. Benito Juárez, 03940, Ciudad de México, México \\ ${ }^{b}$ Departamento de Física, Instituto Nacional de Investigaciones Nucleares, Apartado Postal \\ 18-1027, Col. Escandón, Ciudad de México,11801, México. \\ ${ }^{c}$ Department of Physics, Harvard University, Cambridge, MA 02138, USA \\ ${ }^{d}$ Institute for Computational Cosmology, Department of Physics, Durham University, South \\ Road, Durham DH1 3LE, UK \\ ${ }^{e}$ Department of Astronomy, Cornell University, Ithaca, NY 14853, USA \\ E-mail: avilescervantes@gmail.com, gvalogiannis@g.harvard.edu, \\ marioalberto.rodriguez@inin.gob.mx, jorge.cervantes@inin.gob.mx, \\ baojiu.li@durham.ac.uk, rbean@astro.cornell.edu
}

\begin{abstract}
We develop a framework to compute the redshift space power spectrum (PS), with kernels beyond Einstein-de Sitter (EdS), that can be applied to a wide variety of generalized cosmologies. We build upon a formalism that was recently employed for standard cosmology in Chen, Vlah \& White (2020), and utilize an expansion of the density-weighted velocity moment generating function that explicitly separates the magnitude of the $k$-modes and their angle to the line-of-sight direction dependencies. We compute the PS for matter and biased tracers to 1-loop Perturbation Theory (PT) and show that the expansion has a correct infrared and ultraviolet behavior, free of unwanted divergences. We also add Effective Field Theory (EFT) counterterms, necessary to account for small-scale contributions to PT, and employ an IR-resummation prescription to properly model the smearing of the BAO due to large scale bulk flows within Standard-PT. To demonstrate the applicability of our formalism, we apply it on the $\Lambda \mathrm{CDM}$ and the Hu-Sawicki $f(R)$ models, and compare our numerical results against the ELEPHANT suite of $N$-body simulations, finding very good agreement up to $k=0.27 \mathrm{Mpc}^{-1} h$ at $z=0.5$ for the first three non-vanishing Legendre multipoles of the PS. To our knowledge, the model presented in this work is the most accurate theoretical EFTPT for modified gravity to date, being the only one that accounts for beyond linear local biasing in redshift-space. Hence, we argue our RSD modeling is a promising tool to construct theoretical templates in order to test deviations from $\Lambda \mathrm{CDM}$ using real data obtained from the next stage of cosmological surveys such as DESI and LSST.
\end{abstract}

Keywords: large scale structure formation. perturbation theory. modified gravity. 


\section{Contents}

1 Introduction $\quad 1$

2 Gravity models, fluid equations, and generalized kernels 4

3 Bias expansion $\quad 8$

3.1 Third order bias and renormalization $\quad 11$

$\begin{array}{lll}3.2 & \text { Tracers one-dimensional spectra } & 14\end{array}$

4 Perturbation theory in redshift-space for generalized cosmologies $\quad 15$

$\begin{array}{lll}4.1 & \text { Velocity moments of the generating function } & 16\end{array}$

$\begin{array}{ll}4.2 \text { Computation of moments } & 17\end{array}$

4.3 Moment expansion approach 22

4.4 Large and small scales behavior 24

4.5 EFT counterterms 25

$\begin{array}{lll}4.6 & \text { IR-resummation } & 27\end{array}$

$5 \quad$ Numerical Results $\quad 28$

6 Connection to other works 32

7 Summary and Conclusions $\quad 35$

A $I_{n}^{m}$ functions $\quad 37$

A.1 A function 38

A.2 D function 40

B On the $\mathrm{f}(\mathrm{R})$ Hu-Sawicki model 43

C SPT generalized kernels $\quad 45$

\section{Introduction}

In recent years galaxy and quasar surveys such as 2dF[1], WiggleZ [2], BOSS[3], eBOSS [4] and DES [5] have been measuring statistical properties of the large scale structure of the Universe, and the future is near for more refined, Stage IV probes such as DESI [6], LSST [7], and EUCLID [8]. Certainly, the science of clustering has been boosted over the last two decades and has become essential to extract cosmological information. Two point statistics have been developed in different aspects of theory and numerical simulations, and the successful comparison of both approaches in the region of common validity is an important consistency check to ensure that we are doing well and to gain physical insight into simulation results. Once trustable simulations and mocks are at hand, one is able to compare with observations at the level of precision needed in forthcoming probes, $\sim 1 \%$ or less in the two point statistics observables. 
In perturbation theory (PT) we firstly lead with dark matter density fields, and through bias they connect to tracers such as galaxies. Then, redshift space distortions (RSD), originated by the underlying velocity field along the line of sight to galaxies, are used to measure galaxy clustering [9]. RSD is a solid observable to measure large scale structure, as accounted since the pioneering works of Refs. [10-12] to present.

RSD maps galaxy clustering from real to redshift space, therefore, it introduces an anisotropy to the measured power spectrum (PS) and correlation function, apart from the Alcock-Paczynski anisotropic effect [13], stemming from the coordinate conversion with an incorrect fiducial cosmology. The RSD mapping is nonlinear and intricate due to crosscorrelations between density and velocity fields, but with time advances have been made on its nonlinear modeling, initially carried out by [14-22]. The mapping is cumbersome also because of the Finger-of-God (FoG) feature due to the randomness of the small-scale peculiar velocity field [23]. There is a variety of RSD perturbation models developed in the literature, with the TNS model [22] arguably being one of the most popular choices. Refinements of this model have been put forward, joined by other approaches in a long list of contributions aiming at understanding different issues: the nonlinear mappings and the role of cross correlations between density and velocity fields; the treatment of possible primordial non-Gaussian initial conditions; the regularization of perturbative expansions; effective field theory (EFT) implementations; ways to maximize RSD model performance; applications to biased tracers; and to successfully test models with simulations, among other topics.

RSD measures the growth of structure and therefore can serve as a powerful probe to test gravity. In fact, different clustering measurements have hinted possible deviations from the $\Lambda$ CDM model, assuming WMAP [24] and Planck baseline cosmologies [25-27]. Though deviations are mild and tensions can, in some cases, be attributed to differences in the determination of the clustering amplitude $\left(\sigma_{8}\right)$ between clustering and Planck data, one is motivated to test other gravity models, beyond General Relativity (GR), that at the same time can account for the accelerated expansion of the Universe, whose theoretical origin is still unknown. This is why modified gravity (MG) models could be interesting, among other reasons.

Many studies in MG are based on the linear aspects of the gravitational interactions, since theory gets more complicated than GR. Hence limited models have been often explored in the literature that captures only part of the complexity of MG. In fact when considering perturbations the right-hand-side (RHS) of the Poisson equation is often subject to approximations, such as considering that the effective density perturbation is separable in a function of the wavenumber and scale factor or even that it does not depend on $k$, and that certainly limits the range of possible gravity theories. In a more general scheme, MG induces, even at linear level, scale dependencies and the nonlinear theory becomes cumbersome. In the last years, however, efforts have been made to develop MG cosmological structure formation PT in full generality at one loop in standard perturbation theory (SPT) [28] and in Lagrangian perturbation theory (LPT) [29]. Nonlinear PT is limited to weakly nonlinear scales, but it is nevertheless important because of the baryon acoustic oscillations (BAO) and RSD effects that manifest themselves there. Non-linearities permit us to understand what the important couplings are, among the different Fourier modes that are defined by the kernels [30], and this in turn helps us to understand the role of screening mechanisms in structure formation [31]. These studies have been important to confirm simulation results $[32,33]$ and to construct mocks that employ linear and quasilinear physics analytically [34, 35], and in this way, to achieve the above-mentioned level of precision that new generation of stage IV experiments demand to test gravity [36]. 
RSD in MG has been developed, firstly measuring possible deviations from the growth index and growth rate [37-43], then estimating and testing linear perturbation theory for different MG models [44-47]. RSD nonlinear perturbation theory in MG was built in the context of SPT $[48,49]$ and then in LPT using the Gaussian streaming model [50, 51]. In addition to hybrid approaches combining techniques [52], RSD effects have been also computed through simulations [53-64] and compared to observations [65], finding still modest constraints on the parameter $f_{R 0}\left(<8 \times 10^{-4}\right)$ of the Hu-Sawicki (HS) MG model using the DR11 CMASS data, among other constraints [66, 67]; see however Ref. [68]. Further developments have been made to compute the RSD effect for Vainshtein and Chameleon screening models in the PS [50] and correlation function [50] by numerically computing the SPT kernels using Taruya's method [30]. Although current clustering data is not sufficiently precise to discriminate between different gravity models, the analysis of different systematics is an important task to be better studied. For instance, comparing MG models to observations demands first pipeline validation of theory and simulations, otherwise an issue arises by which GR nuisance parameters and nonlinear RSD modeling can result in biasing MG physical parameters, since there is an overlap of their effects [69-71]. A consistent pipeline treatment and more precise data from upcoming surveys will make possible to disentangle these effects.

Recently a general scheme for RSD has been put forward [72] in which perturbative expansions are expressed in terms of the density-weighted velocity moment generating function, that is based on the pioneering work of [19], which serves to compare in a unified manner the different expansions of RSD modeling and to formulate a Fourier streaming model. This formalism was later applied and tested against simulations in [73] in view of the precision required for the next generation of clustering surveys. We will follow this approach aiming at developing the general theory of RSD for generalized cosmologies to 1-loop approximation. We will find that our expressions are more elaborate because generalized models possess additional scales, and hence, more complex $k$-dependencies. We find generalized kernels, beyond Einstein-de Sitter (EdS), that can be applied to $\Lambda$ CDM, MG models, dark energy with scale-dependent Poisson equation or even massive neutrinos.

In this work we extend the moment expansion approach of [73] to be applied to generalized cosmologies, on which their deviations to $\Lambda$ CDM are encapsulated in the SPT kernels and linear growth function. To do this, we Taylor expand the density-weighted velocity moment generating function accounting for all terms that arise in the 1-loop redshift space PS. In order to model small scales out of the reach of SPT and the effects of the damping of the density and velocity fields along the line-of-sight direction, we utilize the EFT formalism of $[73,74]$. Further, to properly damp the BAO features we use the IR-resummation technique as implemented in [75, 76]. Our tracers are constructed using an extended version of the well-known formalism of [77, 78], that accounts for the additional scales introduced in models beyond GR. Within this work we exemplify our result using the HS $n=1 \mathrm{MG}$ model, for which we present a brief summary in appendix B, focusing on the F6, F5, and F4 realizations. We compare our results for the monopole, quadrupole, and hexadecapole of the spectra against the ELEPHANT MG N-body simulations [79], and find good agreement with our theory up to scales about $k=0.27 \mathrm{Mpc}^{-1} h$ at redshifts $z=0.5$ and $z=1$, for both tracers and matter. To our knowledge, this is the first PT work dealing with the redshift space PS for $\mathrm{MG} /$ generalized cosmologies for tracers beyond linear bias.

The rest of this work is organized as follows: In section 2 we explain the scope of alternative models that are included in our theory and the basics of PT power spectra. In section 3 we present the bias expansion of [77] extended for generalized cosmologies, where 
we also discuss about third order bias and renormalization; in addition, we present here onedimensional spectra for tracers. In section 4 we develop the RSD model, using the moment expansion approach for generalized kernels. We discuss large and small scale behaviors, counterterms from EFT, and use an IR-resummation scheme to properly compute the BAO damping to high- $k$. In section 5 we compute redshift-space power spectra and compare our results to simulations. Complementary information is sent to appendices, where we present expansions of the $I_{n}^{\mathrm{m}}(k)$ functions in Appendix A; formulae for specific MG chosen to present our results, the HS model, in Appendix B, but we emphasize that other MG or alternative models can be straightforwardly employed. The SPT kernels for generalized cosmologies are in Appendix C.

\section{Gravity models, fluid equations, and generalized kernels}

The scope of gravity theories or matter/energy models of the present work are those governed by the Poisson equation that can be written, in Fourier space, as

$$
-\frac{k^{2}}{a^{2}} \Phi(\mathbf{k})=A(k, t) \delta(\mathbf{k})+S(\mathbf{k}),
$$

in which the functions $A(k, t)$ and $S(\mathbf{k})$ determine specific models. Theories such as MG, $k$-dependent dark energy models or massive neutrinos in $\Lambda \mathrm{CDM}$ can be written in this way. To be specific we will exemplify our results, throughout this work, using the $\Lambda$ CDM model and HS $n=1$ F6, F5 and F4 $f(R)$ models; for the latter we present a brief summary in the context of LSS in appendix B. One identifies $A(k, t)$ with the most commonly used $\mu(k, t)$ function through

$$
A(k, t)=A_{0} \mu(k, t), \quad A_{0}=4 \pi G \bar{\rho},
$$

such that these theories can be understood within an effective modification of Newton's constant to $G_{\text {eff }}=\mu(k, t) G$. This function has served to parametrize linear effects of different $\mathrm{MG}$ and dark energy models, but it can be obtained directly from a specific theory.

The function $S$ can also have different origins: In the case of MG models, $S$ comes from the nonlinearities in the Klein-Gordon-like equations and it is responsible for screening mechanisms that drive theories to GR at small scales. In MG theories posed in the Jordan frame it appears sourcing the Poisson equation, as in our eq. (2.1); instead, in theories defined in the Einstein Frame, such as the symmetrons, $S$ appears as a fifth force sourcing geodesic equation, and in this way it will source the Euler equation through the effective gravitational potential $[31,80]$. On the other hand, for massive neutrinos in the $\Lambda$ CDM model it has a special form to account for the nonlinear neutrino density field [81]. Dark energy models can also be accommodated using particular $A(k, t)$ and $S(k)$ functions.

To find $n$-order perturbative solutions, we find $A(k, t)$ analytically (in HS it is given by eq. (B.11)), and assume that $S(\mathbf{k})$ can be expanded in a Taylor series in Fourier space as

$$
S(\mathbf{k})=\frac{1}{2} \int_{\mathbf{k}_{12}=\mathbf{k}} \mathcal{S}^{(2)}\left(\mathbf{k}_{1}, \mathbf{k}_{2}\right) \delta\left(\mathbf{k}_{1}\right) \delta\left(\mathbf{k}_{2}\right)+\frac{1}{6} \int_{\mathbf{k}_{123}=\mathbf{k}} \mathcal{S}^{(3)}\left(\mathbf{k}_{1}, \mathbf{k}_{2}, \mathbf{k}_{3}\right) \delta\left(\mathbf{k}_{1}\right) \delta\left(\mathbf{k}_{2}\right) \delta\left(\mathbf{k}_{3}\right)+\cdots,
$$

whereby the specific $S^{(n)}(k)$ are determined by a particular theory. Note that if $S$ has a linear piece $S=S^{(1)} \delta+\mathcal{O}\left(\delta^{2}\right)$, it can be absorbed by $A(k)$. 
We now turn to the fluid equations, considering a CDM fluid element with peculiar velocity

$$
v^{i}(t)=\frac{d x^{i}(t)}{d \tau}=a \dot{x}^{i}(t)
$$

where $\mathbf{x}$ is its comoving coordinate, $t$ the cosmic time, and $d \tau=\frac{1}{a} d t$ the conformal time, such that the total velocity is $v_{T}^{i}=a H x^{i}+v^{i}$. The fluid equations in the absence of velocity dispersion are

$$
\begin{aligned}
\partial_{t} \delta(\mathbf{x}, t)+\frac{1}{a} \partial_{i}\left[(1+\delta) v^{i}\right] & =0, \\
\partial_{t} v^{i}(\mathbf{x}, t)+\frac{1}{a} v^{j} \partial_{j} v^{i}+H v^{i}+\frac{1}{a} \partial_{i} \Phi & =0,
\end{aligned}
$$

with $\Phi$ the gravitational potential. We use the (dimensionless) velocity divergence

$$
\theta(\mathbf{x}, t)=-\frac{\partial_{i} v^{i}}{a H f_{0}}
$$

where $f_{0}(t)$ is an arbitrary function of time that will be fixed to be the logarithmic growth rate at a convenient scale. We assume the transverse piece of the velocity is negligible at large scales, hence it is a longitudinal field fully specified by $\theta$, hence the above equation can be inverted and $v^{i}(\mathbf{x}, t)=-a H f_{0} \partial_{i} \nabla^{-2} \theta(\mathbf{x}, t)$.

In Fourier space the continuity and Euler equations can be written as ${ }^{1}$

$$
\begin{gathered}
\frac{1}{H} \frac{\partial \delta(\mathbf{k})}{\partial t}-f_{0} \theta(\mathbf{k})=f_{0} \int_{\mathbf{k}_{12}=\mathbf{k}} \alpha\left(\mathbf{k}_{1}, \mathbf{k}_{2}\right) \theta\left(\mathbf{k}_{1}\right) \delta\left(\mathbf{k}_{2}\right), \\
\frac{1}{H} \frac{\partial f_{0} \theta(\mathbf{k})}{\partial t}+\left(2+\frac{\dot{H}}{H^{2}}\right) f_{0} \theta(\mathbf{k})-\frac{A(k)}{H^{2}} \delta(\mathbf{k})-\frac{S(\mathbf{k})}{H^{2}}=f_{0}^{2} \int_{\mathbf{k}_{12}=\mathbf{k}} \beta\left(\mathbf{k}_{1}, \mathbf{k}_{2}\right) \theta\left(\mathbf{k}_{1}\right) \theta\left(\mathbf{k}_{2}\right),
\end{gathered}
$$

with

$$
\alpha\left(\mathbf{k}_{1}, \mathbf{k}_{2}\right)=1+\frac{\mathbf{k}_{1} \cdot \mathbf{k}_{2}}{k_{1}^{2}}, \quad \beta\left(\mathbf{k}_{1}, \mathbf{k}_{2}\right)=\frac{k_{12}^{2}\left(\mathbf{k}_{1} \cdot \mathbf{k}_{2}\right)}{2 k_{1}^{2} k_{2}^{2}} .
$$

One finds perturbative solutions at the different orders according to eqs. $(2.10,2.11)$ and the nonlinear expansion (2.3). To linear order one gets

$$
\begin{aligned}
\delta^{(1)}(\mathbf{k}, t) & =D_{+}(\mathbf{k}, t) \delta^{(1)}\left(\mathbf{k}, t_{0}\right), \\
\theta^{(1)}(\mathbf{k}, t) & =\frac{f(\mathbf{k}, t)}{f_{0}} \delta^{(1)}(\mathbf{k}, t),
\end{aligned}
$$

with $D_{+}$the fastest growing solution to the equation

$$
(\hat{\mathcal{T}}-A(k)) D_{+}(k, t) \equiv\left(\frac{d^{2}}{d t^{2}}+2 H \frac{d}{d t}-A(k, t)\right) D_{+}(k, t)=0,
$$

\footnotetext{
${ }^{1}$ We use the shorthand notations

$$
\int_{\mathbf{p}}=\int \frac{d^{3} p}{(2 \pi)^{3}}
$$

and$$
\int_{\mathbf{k}_{1 \cdots n}=\mathbf{k}}=\int \frac{d^{3} k_{1} \cdots d^{3} k_{n}}{(2 \pi)^{3(n-1)}} \delta_{\mathrm{D}}\left(\mathbf{k}-\mathbf{k}_{1 \cdots n}\right),
$$

with $\mathbf{k}_{1 \cdots n}=\mathbf{k}_{1}+\cdots+\mathbf{k}_{n}$.
} 
where the LHS defines the operator $\hat{\mathcal{T}}$ [82], and

$$
f(k, t)=\frac{d \log D_{+}(k, t)}{d \log a(t)}
$$

is the scale and time dependent growth rate. We choose $f_{0}(t) \equiv f(k=0, t)$, such that at large scales one recovers the linear order solution $\theta=\delta$, valid in $\Lambda \mathrm{CDM}$; which is natural since at very large scales many MG theories reduce to GR, at least in the quasi-static approximation.

In SPT, the $n$-th order velocity and density fields are written as weighted convolutions of $n$ linear density fields,

$$
\begin{aligned}
& \delta^{(n)}(\mathbf{k}, t)=\int_{\mathbf{k}_{1 \cdots n}=\mathbf{k}} F_{n}\left(\mathbf{k}_{1}, \cdots, \mathbf{k}_{n} ; t\right) \delta_{L}\left(\mathbf{k}_{1}, t\right) \cdots \delta_{L}\left(\mathbf{k}_{n}, t\right), \\
& \theta^{(n)}(\mathbf{k}, t)=\int_{\mathbf{k}_{1 \cdots n}=\mathbf{k}} G_{n}\left(\mathbf{k}_{1}, \cdots, \mathbf{k}_{n} ; t\right) \delta_{L}\left(\mathbf{k}_{1}, t\right) \cdots \delta_{L}\left(\mathbf{k}_{n}, t\right)
\end{aligned}
$$

with SPT kernels $F_{n}$ and $G_{n}$ and we have written explicitly their temporal dependence. For linear order, the kernels can be read from eqs. (2.13) and (2.14), giving

$$
F_{1}(\mathbf{k})=1, \quad G_{1}(\mathbf{k})=\frac{f(k)}{f_{0}} .
$$

Higher order kernels can be found by solving eqs. (2.10) and (2.11) iteratively. However, this approach is lengthy, especially for third order kernels, and we found it more efficient to obtain them by means of mappings from LPT known kernels. This is done in appendix C (see also [83]), where we obtain

$$
\begin{aligned}
F_{2}\left(\mathbf{k}_{1}, \mathbf{k}_{2}\right)= & \frac{1}{2}+\frac{3}{14} \mathcal{A}+\left(\frac{1}{2}-\frac{3}{14} \mathcal{B}\right) \frac{\left(\mathbf{k}_{1} \cdot \mathbf{k}_{2}\right)^{2}}{k_{1}^{2} k_{2}^{2}}+\frac{\mathbf{k}_{1} \cdot \mathbf{k}_{2}}{2 k_{1} k_{2}}\left(\frac{k_{2}}{k_{1}}+\frac{k_{1}}{k_{2}}\right) \\
G_{2}\left(\mathbf{k}_{1}, \mathbf{k}_{2}\right)= & \frac{3 \mathcal{A}\left(f_{1}+f_{2}\right)+3 \dot{\mathcal{A}} / H}{14 f_{0}}+\left(\frac{f_{1}+f_{2}}{2 f_{0}}-\frac{3 \mathcal{B}\left(f_{1}+f_{2}\right)+3 \dot{\mathcal{B}} / H}{14 f_{0}}\right) \frac{\left(\mathbf{k}_{1} \cdot \mathbf{k}_{2}\right)^{2}}{k_{1}^{2} k_{2}^{2}} \\
& +\frac{\mathbf{k}_{1} \cdot \mathbf{k}_{2}}{2 k_{1} k_{2}}\left(\frac{f_{2}}{f_{0}} \frac{k_{2}}{k_{1}}+\frac{f_{1}}{f_{0}} \frac{k_{1}}{k_{2}}\right)
\end{aligned}
$$

where $f_{1,2}=f\left(\mathbf{k}_{1,2}\right)$. The functions $\mathcal{A}$ and $\mathcal{B}$ are scale and time dependent:

$$
\mathcal{A}\left(\mathbf{k}_{1}, \mathbf{k}_{2}, t\right)=\frac{7 D_{\mathcal{A}}^{(2)}\left(\mathbf{k}_{1}, \mathbf{k}_{2}, t\right)}{3 D_{+}\left(k_{1}, t\right) D_{+}\left(k_{2}, t\right)}, \quad \mathcal{B}\left(\mathbf{k}_{1}, \mathbf{k}_{2}, t\right)=\frac{7 D_{\mathcal{B}}^{(2)}\left(\mathbf{k}_{1}, \mathbf{k}_{2}, t\right)}{3 D_{+}\left(k_{1}, t\right) D_{+}\left(k_{2}, t\right)},
$$

with second order growth functions $D_{\mathcal{A}, \mathcal{B}}^{(2)}$ are solutions of the linear second order differential equations [29]:

$$
\begin{aligned}
& (\hat{\mathcal{T}}-A(k)) D_{\mathcal{A}}^{(2)}=\left[A(k)+\left(A(k)-A\left(k_{1}\right)\right) \frac{\mathbf{k}_{1} \cdot \mathbf{k}_{2}}{k_{2}^{2}}+\left(A(k)-A\left(k_{2}\right)\right) \frac{\mathbf{k}_{1} \cdot \mathbf{k}_{2}}{k_{1}^{2}}\right. \\
& \left.+\mathcal{S}^{(2)}\left(\mathbf{k}_{1}, \mathbf{k}_{2}\right)\right] D_{+}\left(k_{1}\right) D_{+}\left(k_{2}\right) \\
& (\hat{\mathcal{T}}-A(k)) D_{\mathcal{B}}^{(2)}=\left[A\left(k_{1}\right)+A\left(k_{2}\right)-A(k)\right] D_{+}\left(k_{1}\right) D_{+}\left(k_{2}\right),
\end{aligned}
$$


with appropriate initial conditions to project out the homogeneous first order growth functions, i.e., the kernel of the linear differential operator $(\hat{\mathcal{T}}-A(k))$. Hereafter, when it does not lead to confusion, we omit to write the time dependence of these functions.

Our kernel expressions show where and how the different growth solutions enter to distinguish kernels in different cosmologies. Solutions so far can be specified to the known EdS and $\Lambda$ CDM cases. In fact for $\Lambda \mathrm{CDM}, A(k, t)=A_{0}=\frac{3}{2} \Omega_{m} H^{2}$, and one obtains

$$
D_{\mathcal{A}, \mathcal{B}}^{(2) \Lambda \mathrm{CDM}}(t)=\frac{3}{7} D_{+}^{2}(t)+\frac{4}{7}\left(\hat{\mathcal{T}}-\frac{3}{2} \Omega_{m} H^{2}\right)^{-1}\left[\frac{3}{2} \Omega_{m} H^{2}\left(1-\frac{f^{2}}{\Omega_{m}}\right)\right]
$$

such that $\mathcal{A}^{\Lambda \mathrm{CDM}}=\mathcal{B}^{\Lambda \mathrm{CDM}}$ are only time dependent and close to unity. For EdS, $f=\Omega_{m}=1$, and $\mathcal{A}^{\mathrm{EdS}}=\mathcal{B}^{\mathrm{EdS}}=1$, recovering the EdS standard kernels.

The expressions for $F_{3}$ and $G_{3}$ are large and not displayed here, these are given in Appendix C, eqs. (C.29) and (C.30). Having found the kernels up to third order, we can now construct spectra and cross-spectra of the velocity and density fields. At 1-loop in PT one can use the general expressions

$$
P_{a b}(k)=P_{a b}^{L}(k)+P_{a b}^{22}(k)+P_{a b}^{13}(k),
$$

where $a$ and $b$ refer to $\delta$ or $\theta$ fields, and linear power spectra $P_{a b}^{L}(k)$

$$
P_{\delta \delta}^{L}(k) \equiv P_{L}(k), \quad P_{\delta \theta}^{L}(k)=\frac{f(k)}{f_{0}} P_{L}(k), \quad P_{\theta \theta}^{L}(k)=\left(\frac{f(k)}{f_{0}}\right)^{2} P_{L}(k),
$$

and leading nonlinear contributions

$$
\begin{aligned}
& P_{\delta \delta}^{22}(k)=2 \int_{\mathbf{p}}\left[F_{2}(\mathbf{p}, \mathbf{k}-\mathbf{p})\right]^{2} P_{L}(p) P_{L}(|\mathbf{k}-\mathbf{p}|), \\
& P_{\delta \theta}^{22}(k)=2 \int_{\mathbf{p}} F_{2}(\mathbf{p}, \mathbf{k}-\mathbf{p}) G_{2}(\mathbf{p}, \mathbf{k}-\mathbf{p}) P_{L}(p) P_{L}(|\mathbf{k}-\mathbf{p}|), \\
& P_{\theta \theta}^{22}(k)=2 \int_{\mathbf{p}}\left[G_{2}(\mathbf{p}, \mathbf{k}-\mathbf{p})\right]^{2} P_{L}(p) P_{L}(|\mathbf{k}-\mathbf{p}|), \\
& P_{\delta \delta}^{13}(k)=6 P_{L}(k) \int_{\mathbf{p}} F_{3}(\mathbf{k},-\mathbf{p}, \mathbf{p}) P_{L}(p), \\
& P_{\delta \theta}^{13}(k)=3 P_{L}(k) \int_{\mathbf{p}}\left[F_{3}(\mathbf{k},-\mathbf{p}, \mathbf{p}) G_{1}(\mathbf{k})+G_{3}(\mathbf{k},-\mathbf{p}, \mathbf{p})\right] P_{L}(p), \\
& P_{\theta \theta}^{13}(k)=6 P_{L}(k) \int_{\mathbf{p}} G_{3}(\mathbf{k},-\mathbf{p}, \mathbf{p}) G_{1}(\mathbf{k}) P_{L}(p) .
\end{aligned}
$$

The above expressions are valid for any cosmological model, with the same expressions for power spectra and kernels, the difference being the corresponding growth solutions, and hence the kernels, for each cosmological model. In figure 1 we present our results at 1-loop for the $\Lambda \mathrm{CDM}$ and the F6 HS models, showing their ratios to linear power spectra for cases $a b=\delta \delta, \delta \theta, \theta \theta$; similar results are presented in [50], in which the numerical method of [30] was used to compute the kernels. We note the differences in the gravity models are more pronounced for spectra containing velocity fields, particularly $P_{\theta \theta}$. This is a because even in linear theory velocity fields receive a boost given by the $\frac{f(k)}{f_{0}}$ factors and the relation $\theta^{(1)}=\delta^{(1)}$ does not hold, as it does in $\Lambda$ CDM. Instead, linear fields are related by eq. (2.14), and as a consequence, the differences among models are more pronounced in redshift space than in real space statistics. 

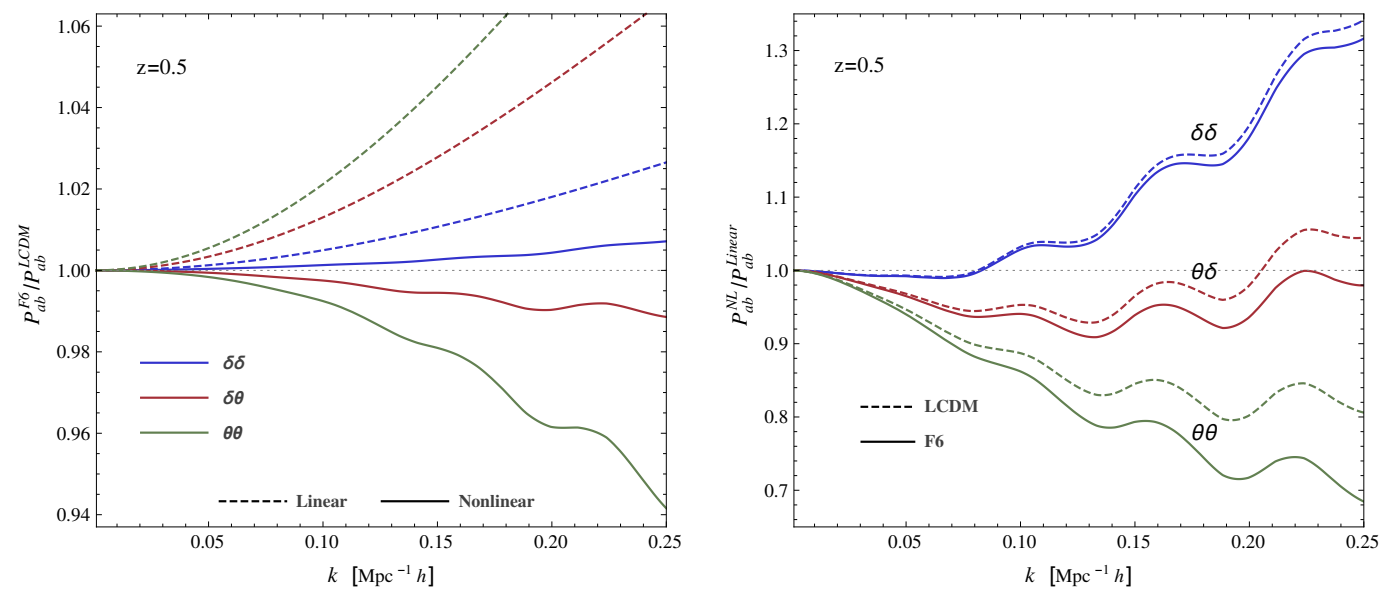

Figure 1. $\quad P_{a b}(k)$ linear and 1-loop power spectra for cases $a b=\delta \delta, \delta \theta, \theta \theta$ at $z=0.5$ computed using eq. (2.26). Left panel: ratios of F6 to $\Lambda$ CDM linear (dashed lines) and non-linear (solid lines) spectra. Right panel: ratios of non-linear to linear power spectra for $\Lambda$ CDM (dashed lines) and F6 (solid lines).

\section{Bias expansion}

This section closely follows the work of McDonald and Roy [77], see also [78, 84], slightly adapted to account for the effects of cosmologies beyond $\Lambda$ CDM. It is well known that for theories with extra degrees of freedom even linear bias becomes scale-dependent, for example in MG [85] or in the presence of massive neutrinos, particularly when biasing the total matter field [86-90]. Our first assumption is the existence of higher-curvature bias operators $\nabla^{2} \delta$, $\nabla^{4} \delta, \ldots$, that effectively encapsulate the effects of a function $A(k)$ that is scale-dependent; see for example section 8 of [91] and [83]. We expand the tracers' density in terms of a set of operators, including the leading curvature operators, labeled with " $m$ " to make reference to matter fields, as

$$
\begin{aligned}
\delta(\mathbf{x})= & c_{\delta} \delta_{m}+c_{\nabla^{2} \delta} \nabla^{2} \delta_{m}+\frac{1}{2} c_{\delta^{2}} \delta_{m}^{2}+\frac{1}{2} c_{s^{2}} s^{2} \\
& +\frac{1}{6} c_{\delta^{3}} \delta_{m}^{3}+\frac{1}{2} c_{\delta s^{2}} \delta s^{2}+c_{\psi} \psi+c_{s t} s t+\frac{1}{2} c_{s^{3}} s^{3} \\
\theta(\mathbf{x})= & \theta_{m}+c_{\nabla^{2} \theta} \nabla^{2} \theta_{m} .
\end{aligned}
$$

We note however that the above bias expansion is not complete since the linear growth function cannot be factorized in time and scale dependent pieces. But by expanding $A(k)$ in powers of $k^{2}$ we can partially tame the new scale introduced in beyond $\Lambda$ CDM models with curvature operators [91]. In the MG models studied here, the parameter expansion is the inverse squared of the mass of the associated scalar field (that can be identified from eq. (B.11) as $m^{2}=M_{1} / 3$ ), so we expect that our modeling better fits for length scales larger than the inverse of this mass [83, 91].

In eq. (3.1), we have used the standard definitions $s^{2}=s_{i j} s_{i j}, s t=s_{i j} t_{i j}, s^{3}=s_{i j} s_{j k} s_{k i}$,

$$
s_{i j}(\mathbf{k})=\left(\frac{k_{i} k_{j}}{k^{2}}-\frac{1}{3} \delta_{i j}\right) \delta_{m}(\mathbf{k}), \quad t_{i j}(\mathbf{k})=\left(\frac{k_{i} k_{j}}{k^{2}}-\frac{1}{3} \delta_{i j}\right) \eta(\mathbf{k})
$$




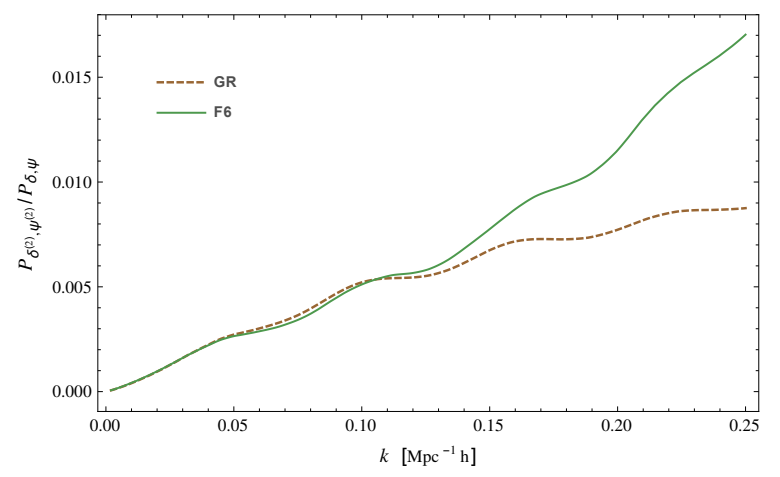

Figure 2. Ratio of $P_{\delta^{(2)} \psi^{(2)}}(k)$ to $P_{\delta \psi}(k)=P_{\delta^{(2)} \psi^{(2)}}(k)+P_{\delta^{(1)} \psi^{(3)}}(k)$ for $\Lambda$ CDM and F6 models at redshift $z=0.5$. This figure shows that the cross contribution of $\psi^{(2)}$ is very small at the scales of interest in PT, about $0.5 \%$ at $k \sim 0.1 \mathrm{~h} / \mathrm{Mpc}$ for both gravity models.

and

$$
\eta(\mathbf{k})=\theta_{m}(\mathbf{k})-\frac{f(k)}{f_{0}} \delta_{m}(\mathbf{k}),
$$

such that in virtue of eq. (2.14), $\eta$ vanishes at linear order in PT. This also means that $t_{i j}$ is second order and hence $s t$ is third order. We further define

$$
\psi(\mathbf{k})=\eta(\mathbf{k})+\frac{f(k)}{f_{0}}\left(-\frac{2}{7} s^{2}(\mathbf{k})+\frac{4}{21} \delta_{m}^{2}(\mathbf{k})\right) .
$$

For EdS kernels, this operator is third order in PT. But even using the $\Lambda$ CDM kernels it is still second order, as we will see below.

We further have considered an operator $\nabla^{2} \theta_{m}$, to be consistent with the inclusion of spatial derivatives of linear overdensities; see section 2.7 in [92]. In the following, we will only keep up to $\nabla^{2} \theta_{m}$ and $\nabla^{2} \delta_{m}$ derivatives and consider them only at the linear level. We do this for simplicity since the addition of higher than second order derivatives complicates largely the algebra and give small contributions; also, although curvature operators are linear, their contributions are of the similar magnitude as those coming from nonlinear operators. Moreover, we will not rely on curvature large bias coefficients for fitting purposes, as for modeling the FoG. Such large contributions, are plausible to come from EFT corrections, which will be introduced in section 4.5 , and are somewhat degenerated with $\nabla^{2}$ biasing, but with a different origin. Hence, we include $\nabla^{2} \theta_{m}$ and $\nabla^{2} \delta_{m}$ biasing operators mainly for consistency and renormalization purposes, and will treat their bias coefficients as small. Otherwise we can always write the tracer PS as an arbitrary polynomial in $k^{2}$ times the matter PS and fit to simulations as much as we want.

The bias expansion in eq. (3.1) can be written as $\delta(\mathbf{x})=\sum c_{\mathcal{O}} \mathcal{O}(\mathbf{x})$, with each operator written in Fourier space at first, second, and third order in PT as

$$
\begin{aligned}
\mathcal{O}^{(1)}(\mathbf{k}) & =K_{\mathcal{O}}^{(1)}(\mathbf{k}) \delta_{m}^{(1)}(\mathbf{k}), \\
\mathcal{O}^{(2)}(\mathbf{k}) & =\int_{\mathbf{k}_{12}=\mathbf{k}} K_{\mathcal{O}}^{(2)}\left(\mathbf{k}_{1}, \mathbf{k}_{2}\right) \delta_{m}^{(1)}\left(\mathbf{k}_{1}\right) \delta_{m}^{(1)}\left(\mathbf{k}_{2}\right), \\
\mathcal{O}^{(3)}(\mathbf{k}) & =\int_{\mathbf{k}_{123}=\mathbf{k}} K_{\mathcal{O}}^{(3)}\left(\mathbf{k}_{1}, \mathbf{k}_{2}, \mathbf{k}_{3}\right) \delta_{m}^{(1)}\left(\mathbf{k}_{1}\right) \delta_{m}^{(1)}\left(\mathbf{k}_{2}\right) \delta_{m}^{(1)}\left(\mathbf{k}_{3}\right) .
\end{aligned}
$$


We now proceed to compute explicitly the $K_{\mathcal{O}}$ kernels for each bias operator order by order:

- The first order contributions are trivially

$$
\begin{aligned}
& \delta^{(1)}(\mathbf{k})=\left(c_{\delta}-c_{\nabla^{2} \delta} k^{2}\right) \delta_{m}^{(1)}(\mathbf{k}), \text { and } \\
& \theta^{(1)}(\mathbf{k})=\left(1-c_{\nabla^{2} \theta} k^{2}\right) \theta_{m}^{(1)}(\mathbf{k}) .
\end{aligned}
$$

- To second order,

$$
\begin{aligned}
& K_{\delta}^{(2)}\left(\mathbf{k}_{1}, \mathbf{k}_{2}\right)=F_{2}\left(\mathbf{k}_{1}, \mathbf{k}_{2}\right) \\
& K_{\delta^{2}}^{(2)}\left(\mathbf{k}_{1}, \mathbf{k}_{2}\right)=1 \\
& K_{s^{2}}^{(2)}\left(\mathbf{k}_{1}, \mathbf{k}_{2}\right)=S_{2}\left(\mathbf{k}_{1}, \mathbf{k}_{2}\right), \\
& K_{\psi}^{(2)}\left(\mathbf{k}_{1}, \mathbf{k}_{2}\right)=G_{2}\left(\mathbf{k}_{1}, \mathbf{k}_{2}\right)-G_{1}\left(\mathbf{k}_{12}\right)\left[F_{2}\left(\mathbf{k}_{1}, \mathbf{k}_{2}\right)+\frac{2}{7} S_{2}\left(\mathbf{k}_{1}, \mathbf{k}_{2}\right)-\frac{4}{21}\right],
\end{aligned}
$$

with

$$
S_{2}\left(\mathbf{k}_{1}, \mathbf{k}_{2}\right)=\frac{\left(\mathbf{k}_{1} \cdot \mathbf{k}_{2}\right)^{2}}{k_{1}^{2} k_{2}^{2}}-\frac{1}{3} .
$$

Note that $G_{1}^{\mathrm{EdS}}=1$, and $\frac{2}{7} S_{2}(\mathbf{k}, \mathbf{p})-\frac{4}{21}=G_{2}^{\mathrm{EdS}}(\mathbf{k}, \mathbf{p})-F_{2}^{\mathrm{EdS}}(\mathbf{k}, \mathbf{p})$, and hence for EdS kernels the operator $\psi$ vanishes at second order. However, if $\Lambda$ CDM kernels are used, we have instead

$$
K_{\psi}^{(2), \Lambda \mathrm{CDM}}\left(\mathbf{k}_{1}, \mathbf{k}_{2}\right)=\frac{3}{14}\left(\mathcal{A}^{\Lambda \mathrm{CDM}}-1-\frac{\dot{\mathcal{A}}^{\Lambda \mathrm{CDM}}}{f_{0} H}\right)\left(1-\frac{\left(\mathbf{k}_{1} \cdot \mathbf{k}_{2}\right)^{2}}{k_{1}^{2} k_{2}^{2}}\right),
$$

so the operator $\psi$ vanishes only at first order in $\mathrm{PT}$ as we commented above. Despite $\psi$ being second order, we will treat it as third order because its influence is indeed small, as can be seen in figure 2 , where we show the cross-power spectrum $P_{\delta^{(2)} \psi^{(2)}}(k)=$ $\left\langle\delta^{(2)}(\mathbf{k}) \psi^{(2)}\left(\mathbf{k}^{\prime}\right)\right\rangle^{\prime}$ divided by the full spectrum $P_{\delta \psi}(k)=\left\langle\delta(\mathbf{k}) \psi\left(\mathbf{k}^{\prime}\right)\right\rangle^{\prime}$, which also receives a contribution from $\psi$ at third order, $\left\langle\delta^{(1)}(\mathbf{k}) \psi^{(3)}\left(\mathbf{k}^{\prime}\right)\right\rangle^{\prime}$. We do this for $\Lambda$ CDM and F6 noticing that the error introduced by neglecting the $P_{\delta^{(2)} \psi^{(2)}}(k)$ piece is about the $1 \%$ or smaller for $k \lesssim 0.2 \mathrm{hMpc}^{-1}$ for both models. This suggests to make $\psi^{(2)}=0$, as we do in the following. In $\Lambda \mathrm{CDM}$, regardless of $\psi$ being second order, one can absorb the residual given by eq. (3.16) in the bias parameters $c_{s^{2}}$ and $c_{\delta^{2}}$ in virtue of eqs. (3.12) and (3.13). Alternatively, $\psi$ can be easily redefined to be third order, as in [93]. 
- Third order kernels are

$$
\begin{aligned}
K_{\delta}^{(3)}\left(\mathbf{k}_{1}, \mathbf{k}_{2}, \mathbf{k}_{3}\right)= & F_{3}\left(\mathbf{k}_{1}, \mathbf{k}_{2}, \mathbf{k}_{3}\right), \\
K_{\delta^{2}}^{(3)}\left(\mathbf{k}_{1}, \mathbf{k}_{2}, \mathbf{k}_{3}\right)= & 2 F_{2}\left(\mathbf{k}_{2}, \mathbf{k}_{3}\right) \\
K_{\delta^{3}}^{(3)}\left(\mathbf{k}_{1}, \mathbf{k}_{2}, \mathbf{k}_{3}\right)= & 1 \\
K_{s^{2}}^{(3)}\left(\mathbf{k}_{1}, \mathbf{k}_{2}, \mathbf{k}_{3}\right)= & 2 S_{2}\left(\mathbf{k}_{1}, \mathbf{k}_{2}+\mathbf{k}_{3}\right) F_{2}\left(\mathbf{k}_{2}, \mathbf{k}_{3}\right), \\
K_{\delta s^{2}}^{(3)}\left(\mathbf{k}_{1}, \mathbf{k}_{2}, \mathbf{k}_{3}\right)= & S_{2}\left(\mathbf{k}_{1}, \mathbf{k}_{2}\right) \\
K_{s^{3}}^{(3)}\left(\mathbf{k}_{1}, \mathbf{k}_{2}, \mathbf{k}_{3}\right)= & S_{3}\left(\mathbf{k}_{1}, \mathbf{k}_{2}, \mathbf{k}_{3}\right), \\
K_{s t}^{(3)}\left(\mathbf{k}_{1}, \mathbf{k}_{2}, \mathbf{k}_{3}\right)= & S_{2}\left(\mathbf{k}_{1}, \mathbf{k}_{2}+\mathbf{k}_{3}\right)\left(G_{2}\left(\mathbf{k}_{2}, \mathbf{k}_{3}\right)-\frac{f\left(\left|\mathbf{k}_{23}\right|\right)}{f_{0}} F_{2}\left(\mathbf{k}_{2}, \mathbf{k}_{3}\right)\right), \\
K_{\psi}^{(3)}\left(\mathbf{k}_{1}, \mathbf{k}_{2}, \mathbf{k}_{3}\right)= & G_{3}\left(\mathbf{k}_{1}, \mathbf{k}_{2}, \mathbf{k}_{3}\right)-G_{1}\left(\mathbf{k}_{123}\right) F_{3}\left(\mathbf{k}_{1}, \mathbf{k}_{2}, \mathbf{k}_{3}\right) \\
& -2 G_{1}\left(\mathbf{k}_{123}\right) F_{2}\left(\mathbf{k}_{2}, \mathbf{k}_{3}\right)\left(\frac{2}{7} S_{2}\left(\mathbf{k}_{1}, \mathbf{k}_{2}+\mathbf{k}_{3}\right)-\frac{4}{21}\right),
\end{aligned}
$$

with

$$
S_{3}\left(\mathbf{k}_{1}, \mathbf{k}_{2}, \mathbf{k}_{3}\right)=\left(\frac{k_{1}^{i} k_{1}^{j}}{k_{1}^{2}}-\frac{1}{3} \delta_{i j}\right)\left(\frac{k_{2}^{j} k_{2}^{k}}{k_{2}^{2}}-\frac{1}{3} \delta_{j k}\right)\left(\frac{k_{3}^{k} k_{3}^{i}}{k_{3}^{2}}-\frac{1}{3} \delta_{k i}\right) .
$$

Note also that $\mathcal{A}(\mathbf{p},-\mathbf{p})=\mathcal{B}(\mathbf{p},-\mathbf{p})$, as implied from eqs. (2.23) and (2.24), and by means that the source vanishes at very large scales $S(\mathbf{k}=0)=0$. Therefore, $F_{2}(\mathbf{p},-\mathbf{p})=0$; more generally, we have $F_{2}(\mathbf{p}, \mathbf{q} \rightarrow-\mathbf{p}) \propto|\mathbf{p}+\mathbf{q}|^{2}$, which is a consequence of momentum conservation.

\subsection{Third order bias and renormalization}

It is standard to define the function $[77,78]$

$$
\sigma_{3}^{2}(k)=\frac{105}{16} \int_{\mathbf{p}} P_{L}(p)\left[S_{2}(\mathbf{p}, \mathbf{k}-\mathbf{p})\left(\frac{2}{7} S_{2}(-\mathbf{p}, \mathbf{k})-\frac{4}{21}\right)+\frac{8}{63}\right],
$$

that serves to collect some of the biasing terms constructed out from operators at third order in PT. Here, we outline the procedure to do that. First consider the linear matter overdensity field $\delta_{m}^{(1)}$ correlated with operators $s t, s^{2}$ and $\psi$, yielding the spectra $P_{\delta_{m}^{(1)}, s t^{(3)}}=$ $\left\langle\delta_{m}^{(1)}(\mathbf{k}) s t^{(3)}\left(\mathbf{k}^{\prime}\right)\right\rangle^{\prime}, P_{\delta_{m}^{(1)}, s^{2(3)}}=\left\langle\delta_{m}^{(1)}(\mathbf{k}) s^{2(3)}\left(\mathbf{k}^{\prime}\right)\right\rangle^{\prime}$, and $P_{\delta_{m}^{(1)}, \psi^{(3)}}=\left\langle\delta_{m}^{(1)}(\mathbf{k}) \psi^{(3)}\left(\mathbf{k}^{\prime}\right)\right\rangle^{\prime}$, contribut- 
ing to the matter-tracer cross-power spectrum as

$$
\begin{aligned}
P(k) & \ni \frac{1}{2} c_{s^{2}} P_{\delta_{m}^{(1)}, s^{2(3)}}(k)+c_{s t} P_{\delta_{m}^{(1)}, s t^{(3)}}(k)+c_{\psi} P_{\delta_{m}^{(1)}, \psi^{(3)}}(k) \\
& =c_{s}^{2} P_{L}(k) \int_{\mathbf{p}} P_{L}(p)\left[K_{s^{2}}^{(3)}(\mathbf{p},-\mathbf{p}, \mathbf{k})-K_{s^{2}}^{(3)}(\mathbf{p},-\mathbf{p}, 0)\right] \\
& +2 c_{s t} P_{L}(k) \int_{\mathbf{p}} P_{L}(p)\left[K_{s t}^{(3)}(\mathbf{p},-\mathbf{p}, \mathbf{k})-K_{s t}^{(3)}(\mathbf{p},-\mathbf{p}, 0)\right] \\
+ & 2 c_{\psi} P_{L}(k) \int_{\mathbf{p}} P_{L}(p)\left\{\frac{3}{2}\left[G_{3}(\mathbf{k},-\mathbf{p}, \mathbf{p})-\frac{f(k)}{f_{0}} F_{3}(\mathbf{k},-\mathbf{p}, \mathbf{p})\right]\right. \\
& \left.-2 \frac{f(k)}{f_{0}} F_{2}(-\mathbf{p}, \mathbf{k})\left(\frac{2}{7} S(\mathbf{p}, \mathbf{k}-\mathbf{p})-\frac{4}{21}\right)\right\} .
\end{aligned}
$$

A direct computation does not give the terms $K_{s^{2}}^{(3)}(\mathbf{p},-\mathbf{p}, 0)$ and $K_{s t}^{(3)}(\mathbf{p},-\mathbf{p}, 0)$. These should be included in order to make the large scales insensitive to the small scales, otherwise when these integrals are regularized, for example by a sharp cutoff at $p=\Lambda \gg 1$, they go as $\Lambda^{3+n}$ for a scale invariant PS $P_{L}(k \gg 1) \propto k^{n}$, becoming UV divergent for a typical PS. More explicitly, these terms are

$$
\begin{gathered}
\int_{\mathbf{p}} P_{L}(p) K_{s^{2}}^{(3)}(\mathbf{p},-\mathbf{p}, 0)=\int_{\mathbf{p}} P_{L}(p)\left[\frac{8}{9}+\frac{2}{21}(3 \mathcal{A}(0, \mathbf{p})-\mathcal{B}(0, \mathbf{p}))\right]=\frac{68}{63} \mathcal{C}_{s^{2}} \sigma^{2} \\
\int_{\mathbf{p}} P_{L}(p) K_{s t}^{(3)}(\mathbf{p},-\mathbf{p}, 0)=\int_{\mathbf{p}} P_{L}(p)\left\{\frac{1}{9}-\frac{3 f(p)}{9 f_{0}}+\frac{f(p)(3 \mathcal{A}(0, \mathbf{p})-\mathcal{B}(0, \mathbf{p}))}{21 f_{0}}\right. \\
\left.+\frac{3 \dot{\mathcal{A}}(0, \mathbf{p})-\dot{\mathcal{B}}(0, \mathbf{p})}{21 f_{0} H}\right\}=-\frac{8}{63} \mathcal{C}_{s t} \sigma^{2}
\end{gathered}
$$

For large values of the inner integration moment $\mathbf{p}$, both $\mathcal{A}(0, \mathbf{p})$ and $\mathcal{B}(0, \mathbf{p})$ converge (this is for the case in which function $\mu(k)$ is bounded), hence $\mathcal{C}_{s^{2}}$ and $\mathcal{C}_{s t}$ are constants. For $\Lambda$ CDM kernels, $\mathcal{C}_{s^{2}}=\frac{63}{68}\left[\frac{8}{9}+\frac{4}{21} \mathcal{A}^{\Lambda \mathrm{CDM}}\right]$ and $\mathcal{C}_{s t}=\frac{63}{8}\left[\frac{2}{9}-\frac{2}{21}\left(\mathcal{A}^{\Lambda \mathrm{CDM}}+\dot{\mathcal{A}}^{\Lambda \mathrm{CDM}} / f_{0} H\right)\right]$, and both reducing to 1 for EdS kernels. These $\mathbf{k} \rightarrow 0$ terms contribute to the PS as $\propto \sigma^{2} P_{L}(k)$, so they can be absorbed by the linear bias parameter by redefining it as $c_{\delta} \rightarrow c_{\delta}-\frac{68}{63} \mathcal{C}_{s^{2}} \sigma^{2}+\frac{8}{63} \mathcal{C}_{s t} \sigma^{2}$.

We introduce the functions

$$
\begin{aligned}
\sigma_{3, s^{2}}^{2}(k) & =-\frac{21}{16} \int_{\mathbf{p}} P_{L}(p)\left[K_{s^{2}}^{(3)}(\mathbf{p},-\mathbf{p}, \mathbf{k})-K_{s^{2}}^{(3)}(\mathbf{p},-\mathbf{p}, 0)\right] \\
\sigma_{3, s t}^{2}(k) & =\frac{105}{16} \int_{\mathbf{p}} P_{L}(p)\left[K_{s t}^{(3)}(\mathbf{p},-\mathbf{p}, \mathbf{k})-K_{s t}^{(3)}(\mathbf{p},-\mathbf{p}, 0)\right] \\
\sigma_{3, \psi}^{2}(k) & =\frac{2205}{256} \int_{\mathbf{p}} P_{L}(p)\left\{\frac{3}{2}\left[G_{3}(\mathbf{k},-\mathbf{p}, \mathbf{p})-\frac{f(k)}{f_{0}} F_{3}(\mathbf{k},-\mathbf{p}, \mathbf{p})\right]\right. \\
& \left.-2 \frac{f(k)}{f_{0}} F_{2}(-\mathbf{p}, \mathbf{k})\left(\frac{2}{7} S_{2}(\mathbf{p}, \mathbf{k}-\mathbf{p})-\frac{4}{21}\right)\right\}
\end{aligned}
$$

For EdS kernels, these three functions are identical to $\sigma_{3}^{2}(k)$, given above in eq. (3.26), and hence all the biasing terms involving operators $s^{2}$, st and $\psi$ at third order can be grouped 
into a single one, contributing to the PS as $b_{3 n l} \sigma_{3}^{2}(k) P_{L}(k) .{ }^{2}$ In $\Lambda \mathrm{CDM}$, the $k$-dependence of these functions is the same as well, proportional to $\sigma_{3}^{2}(k)$, differing only by multiplicative constants, which are irrelevant because they can be absorbed by their own bias parameters. In generalized cosmologies, instead, not only the normalization is different, but also the $k$ dependence. At very large scales the $\sigma_{3,\left\{s^{2}, s t, \psi\right\}}^{2}(k)$ functions have the same $k$-dependence, but they differ significantly at smaller, but yet linear scales. In figure 3 we plot these functions for the F6 model at $z=0.5$ normalized to have the same large scales values and divided by the EdS $\sigma_{3}^{2}(k)$, showing significant differences between them even at linear scales. The most straightforward route we can follow is to consider the three contributions separately with three different bias parameters $c_{s^{2}}, c_{s t}$, and $c_{\psi}$. However, we note that in general we can approximate

$$
\sigma_{3,\left\{s^{2}, s t, \psi\right\}}^{2}(k) \approx \sigma_{3}^{2}(k)-\alpha_{1,\left\{s^{2}, s t, \psi\right\}} k^{2}-\alpha_{2,\left\{s^{2}, s t, \psi\right\}} k^{4}+\cdots
$$

as much as we desire over a given finite interval, and absorb $\alpha_{1,\left\{s^{2}, s t, \psi\right\}} k^{2}, \alpha_{2,\left\{s^{2}, s t, \psi\right\}} k^{4}, \ldots$ into the higher-order curvature biases $c_{\nabla^{2} \delta}, c_{\nabla^{4} \delta}, \ldots$ Then, we write

$$
\frac{1}{2} c_{s^{2}} P_{\delta_{m}^{(1)}, s^{2(3)}}(k)+c_{s t} P_{\delta_{m}^{(1)}, s t^{(3)}}(k)+c_{\psi} P_{\delta_{m}^{(1)}, \psi^{(3)}}(k)=b_{3 n l} \sigma_{3}^{2}(k) P_{L}(k)
$$

with

$$
b_{3 n l}=\frac{32}{105} c_{s t}-\frac{16}{21} c_{s^{2}}+\frac{512}{2205} c_{\psi},
$$

together with the redefinitions

$$
\begin{aligned}
c_{\delta} & \longrightarrow c_{\delta}-\frac{68}{63} \mathcal{C}_{s^{2}} \sigma^{2}+\frac{16}{63} \mathcal{C}_{s t} \sigma^{2}, \\
c_{\nabla^{2 m} \delta} & \longrightarrow c_{\nabla^{2 m} \delta}-\frac{32}{105} c_{s t} \alpha_{m, s t}+\frac{16}{21} c_{s^{2}} \alpha_{m, s^{2}}-\frac{512}{2205} c_{\psi} \alpha_{m, \psi} .
\end{aligned}
$$

In figure 3 we also show the curves for these functions once corrected by a polynomial in $k^{2}$ that we take linear, i.e., we plot (dashed lines) $\sigma_{3,\left\{s^{2}, s t, \psi\right\}}^{2}(k)+\alpha_{1,\left\{s^{2}, s t, \psi\right\}} k^{2}$ divided by $\sigma_{3}^{2}(k)$, showing an agreement of less than $2-3 \%$ over the interval $k \in\{0,0.2\}$, covering the mild non-linear scales reached by PT. Clearly as higher degree polynomials are considered, a better agreement we can get; however, this requires to add the corresponding higher-order bias operators into the theory. Here, we opted to include only the first curvature bias $\nabla^{2} \delta_{m}$.

The computation of power spectra involving a linear matter velocity field is almost identical since $\left\langle\theta_{m}^{(1)}(\mathbf{k}) \mathcal{O}^{(3)}\left(\mathbf{k}^{\prime}\right)\right\rangle=\frac{f(k)}{f_{0}}\left\langle\delta_{m}^{(1)}(\mathbf{k}) \mathcal{O}^{(3)}\left(\mathbf{k}^{\prime}\right)\right\rangle$. Hence, we only have to keep track of the $f(k) / f_{0}$ factors: instead of eq. (3.34), we obtain

$$
\frac{1}{2} c_{s^{2}} P_{\theta_{m}^{(1)}, s^{2(3)}}(k)+c_{s t} P_{\theta_{m}^{(1)}, t^{(3)}}(k)+c_{\psi} P_{\theta_{m}^{(1)}, \psi^{(3)}}(k)=b_{3 n l} \sigma_{3}^{2}(k) \frac{f(k)}{f_{0}} P_{L}(k) .
$$

and the polynomials in $k^{2}$ should be absorbed by higher-order velocity derivative bias parameters

$$
c_{\nabla^{2} \theta} \longrightarrow c_{\nabla^{2} \theta}-\frac{32}{105} c_{s t} \alpha_{1, s t}+\frac{16}{21} c_{s^{2}} \alpha_{1, s^{2}}-\frac{512}{2205} c_{\psi} \alpha_{1, \psi}
$$

\footnotetext{
${ }^{2}$ There are other three spectra of the form $\left\langle\delta_{m}^{(1)} \mathcal{O}^{(3)}\right\rangle$ involving an operator at third order: $\left\langle\delta(\mathbf{k}) s^{3}\left(\mathbf{k}^{\prime}\right)\right\rangle^{\prime}=0$, so $s^{3}$ does not contribute to 1-loop PS; $\left\langle\delta(\mathbf{k}) \delta^{3}\left(\mathbf{k}^{\prime}\right)\right\rangle^{\prime} \propto \sigma^{2} P_{L}$, so it is UV sensitive but absorbed by linear bias parameter; and $\left\langle\delta(\mathbf{k}) \delta^{2(3)}\left(\mathbf{k}^{\prime}\right)\right\rangle^{\prime}$, which is treated separately and contributes to the term $P_{b_{1} b_{2}}$ in eq. (3.40) below.
} 


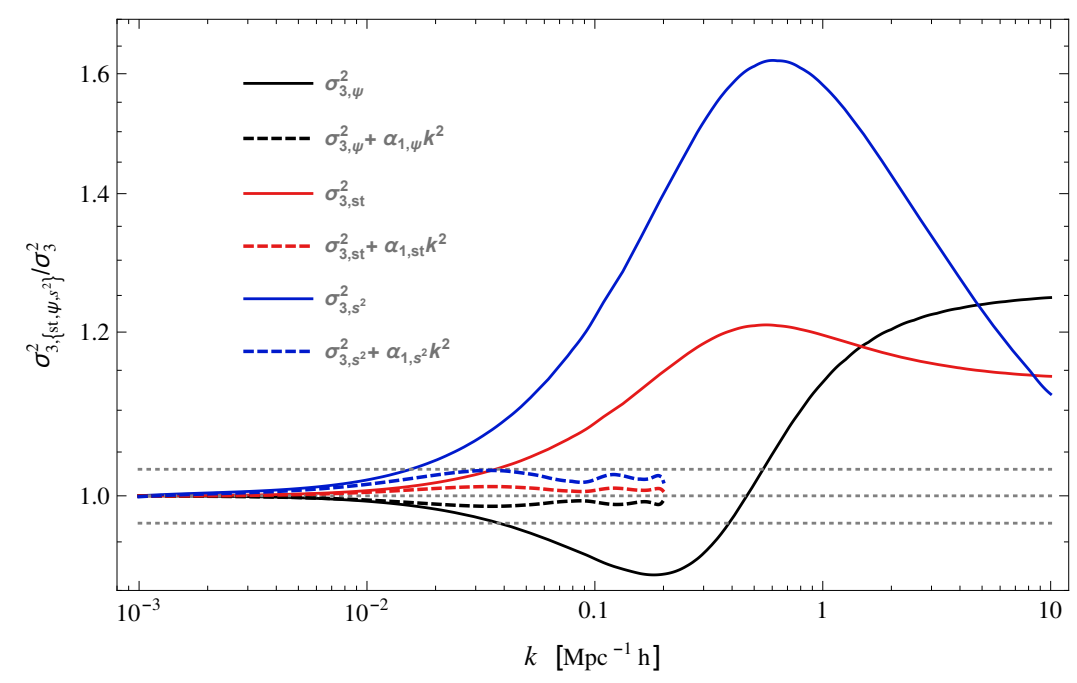

Figure 3. $\sigma_{3}^{2}$ functions. Solid lines show the ratio of biasing functions $\sigma_{3,\left\{s^{2}, s t, \psi\right\}}^{2}(k)$ in the F6 model with respect to $\sigma_{3}^{2}$ EdS given by eq. (3.26). Dashed lines show the same as solid's, but now correcting the functions with a quadratic term $k^{2}$ that becomes absorbed by higher curvature bias.

We emphasize that collecting some of the biasing terms into $\sigma_{3}^{2}(k)$ is not necessary on theoretical grounds, but is adopted here in order to keep as less bias parameters as possible.

The above treatment completes the biasing formalism adopted in this work, valid for $\Lambda \mathrm{CDM}$ and alternative cosmological models.

\subsection{Tracers one-dimensional spectra}

We now compute the PS for biased tracers using the power spectra expressions at the end of section 2, but for tracers developed in the previous subsection. The spectra $P_{\delta \delta}, P_{\delta \theta}$, and $P_{\theta \theta}$ are computed using the standard tools developed in [77], see also [78], obtaining

$$
\begin{aligned}
P_{\delta \delta}(k)= & \left(b_{1}-b_{\nabla^{2} \delta} k^{2}\right)^{2} P_{L}(k)+b_{1}^{2} P_{m, \delta \delta}^{\mathrm{loop}}(k)+2 b_{1} b_{2} P_{b_{1} b_{2}}(k)+2 b_{1} b_{s^{2}} P_{b_{1} b_{s^{2}}}(k) \\
& +b_{2}^{2} P_{b_{2}^{2}}(k)+2 b_{2} b_{s^{2}} P_{b_{2} b_{s^{2}}}(k)+b_{s^{2}}^{2} P_{b_{s^{2}}}(k)+2 b_{1} b_{3 n l} \sigma_{3}^{2}(k) P_{L}(k), \\
P_{\delta \theta}(k)= & \left(b_{1}-b_{\nabla^{2} \delta} k^{2}\right)\left(1-b_{\nabla^{2} \theta} k^{2}\right) \frac{f(k)}{f_{0}} P_{L}(k)+b_{1} P_{m, \delta \theta}^{\mathrm{loop}}(k)+b_{2} P_{b_{2}, \theta}(k) \\
& +b_{s^{2}} P_{b_{s^{2}}, \theta}(k)+b_{3 n l} \sigma_{3}^{2}(k) \frac{f(k)}{f_{0}} P_{L}(k), \\
P_{\theta \theta}(k)= & \left(1-b_{\nabla^{2} \theta} k^{2}\right)^{2}\left(\frac{f(k)}{f_{0}}\right)^{2} P_{L}(k)+P_{m, \theta \theta}^{\mathrm{loop}}(k),
\end{aligned}
$$


with

$$
\begin{aligned}
P_{b_{1} b_{2}}(k) & =\int_{\mathbf{k}_{12}=\mathbf{k}} F_{2}\left(\mathbf{k}_{1}, \mathbf{k}_{2}\right) P_{L}\left(\mathbf{k}_{1}\right) P_{L}\left(\mathbf{k}_{2}\right), \\
P_{b_{1} b_{s^{2}}}(k) & =\int_{\mathbf{k}_{12}=\mathbf{k}} F_{2}\left(\mathbf{k}_{1}, \mathbf{k}_{2}\right) S_{2}\left(\mathbf{k}_{1}, \mathbf{k}_{2}\right) P_{L}\left(\mathbf{k}_{1}\right) P_{L}\left(\mathbf{k}_{2}\right), \\
P_{b_{2}^{2}}(k) & =\frac{1}{2} \int_{\mathbf{k}_{12}=\mathbf{k}} P_{L}\left(\mathbf{k}_{1}\right)\left[P_{L}\left(\mathbf{k}_{2}\right)-P_{L}\left(\mathbf{k}_{1}\right)\right], \\
P_{b_{2} b_{s^{2}}}(k) & =\frac{1}{2} \int_{\mathbf{k}_{12}=\mathbf{k}} P_{L}\left(\mathbf{k}_{1}\right)\left[P_{L}\left(\mathbf{k}_{2}\right) S_{2}\left(\mathbf{k}_{1}, \mathbf{k}_{2}\right)-\frac{2}{3} P_{L}\left(\mathbf{k}_{1}\right)\right], \\
P_{b_{s^{2}}}(k) & =\frac{1}{2} \int_{\mathbf{k}_{12}=\mathbf{k}} P_{L}\left(\mathbf{k}_{1}\right)\left[P_{L}\left(\mathbf{k}_{2}\right)\left[S_{2}\left(\mathbf{k}_{1}, \mathbf{k}_{2}\right)\right]^{2}-\frac{4}{9} P_{L}\left(\mathbf{k}_{1}\right)\right],
\end{aligned}
$$

and

$$
\begin{aligned}
P_{b_{2}, \theta}(k) & =\int_{\mathbf{k}_{12}=\mathbf{k}} G_{2}\left(\mathbf{k}_{1}, \mathbf{k}_{2}\right) P_{L}\left(\mathbf{k}_{1}\right) P_{L}\left(\mathbf{k}_{2}\right) \\
P_{b_{s^{2}}, \theta}(k) & =\int_{\mathbf{k}_{12}=\mathbf{k}} G_{2}\left(\mathbf{k}_{1}, \mathbf{k}_{2}\right) S_{2}\left(\mathbf{k}_{1}, \mathbf{k}_{2}\right) P_{L}\left(\mathbf{k}_{1}\right) P_{L}\left(\mathbf{k}_{2}\right),
\end{aligned}
$$

with renormalized bias parameters

$$
b_{1}=c_{\delta}+\left[\frac{31}{24} c_{\delta^{2}} \mathcal{C}_{\delta^{2}}+\frac{1}{2} c_{\delta^{3}} \mathcal{C}_{\delta^{3}}+\frac{1}{3} c_{\delta s^{2}} \mathcal{C}_{\delta s^{2}}+\frac{68}{63} c_{s^{2}} \mathcal{C}_{s^{2}}-\frac{16}{63} c_{s t} \mathcal{C}_{s t}\right] \sigma^{2},
$$

with $\mathcal{C}_{\mathcal{O}}$ constants of order unity and they are required in cosmologies beyond EdS, as we have seen for $\mathcal{C}_{s^{2}}$ and $\mathcal{C}_{s t}$. At 1-loop in the PS the rest of biasing parameters remain equal: $b_{2}=c_{\delta^{2}}$ and $b_{s^{2}}=c_{s^{2}}$.

\section{Perturbation theory in redshift-space for generalized cosmologies}

As we observe objects in the sky we map them through their angular position $\hat{\mathbf{n}}$ and radial position as inferred from their redshift. The latter is given by the Hubble flow and their peculiar velocity $\mathbf{v}$. Hence, an object located at a comoving distance $\mathbf{x}$ is observed to be at an apparent position $\mathbf{s}$, such that the map between real and redshift space positions is given by the non-relativistic, longitudinal Doppler effect,

$$
\mathbf{s}=\mathbf{x}+\mathbf{u},
$$

with line-of-sight "velocity" $\mathbf{u}$ defined as

$$
\mathbf{u} \equiv \hat{\mathbf{n}} \frac{\mathbf{v} \cdot \hat{\mathbf{n}}}{a H} .
$$

We use the plane-parallel approximation, on which $\hat{\mathbf{n}}$ is a constant vector pointing in the direction of the objects sample, instead of being equal to the position unit vector $\hat{\mathbf{x}}$. We 
also assume that the velocity is longitudinal, with divergence field $\theta$, defined in eq. (2.7), for which

$$
\mathbf{u}(\mathbf{k})=i f_{0} \hat{\mathbf{n}} \frac{\mathbf{k} \cdot \hat{\mathbf{n}}}{k^{2}} \theta(\mathbf{k}),
$$

that we will mostly use.

Clearly, the map to redshift coordinates conserves the number of tracers, $\left[1+\delta_{s}(\mathbf{s})\right] d^{3} s=$ $[1+\delta(\mathbf{x})] d^{3} x$, yielding

$$
(2 \pi)^{3} \delta_{\mathrm{D}}(\mathbf{k})+\delta_{s}(\mathbf{k})=\int d^{3} x(1+\delta(\mathbf{x})) e^{-i \mathbf{k} \cdot(\mathbf{x}+\mathbf{u}(\mathbf{x}))},
$$

and the redshift-space PS becomes [19, 72]

$$
(2 \pi)^{3} \delta_{\mathrm{D}}(\mathbf{k})+P_{s}(\mathbf{k})=\int d^{3} x e^{-i \mathbf{k} \cdot \mathbf{x}}[1+\mathcal{M}(\mathbf{J}=\mathbf{k}, \mathbf{x})],
$$

with velocity moments generating function

$$
1+\mathcal{M}(\mathbf{J}, \mathbf{x})=\left\langle\left(1+\delta\left(\mathbf{x}_{1}\right)\right)\left(1+\delta\left(\mathbf{x}_{2}\right)\right) e^{-i \mathbf{J} \cdot \Delta \mathbf{u}}\right\rangle,
$$

where $\Delta \mathbf{u}=\mathbf{u}\left(\mathbf{x}_{2}\right)-\mathbf{u}\left(\mathbf{x}_{1}\right)$ and $\mathbf{x}=\mathbf{x}_{2}-\mathbf{x}_{1}$. Function $\mathcal{M}$ (or its Fourier transform) plays a central role in RSD. Different expansion procedures of eq. (4.6) yield different approaches to RSD modeling, grouped in [72] as: direct Lagrangian, moment expansion, streaming model, and smoothing kernel. We will follow here the moment expansion approach, in which the exponential in the generating function is expanded and the moments are evaluated. Thereafter we will consider EFT contributions which yield the damping along the line-of-sight direction produced by non-coherent motions of particles at small scales. The connection to smoothing kernels will be discussed in section 6 .

\subsection{Velocity moments of the generating function}

The m-th density weighted velocity field moment of the generating function is an m-rank tensor defined as [19, 72]

$$
\left.\Xi_{i_{1} \cdots i_{\mathrm{m}}}^{\mathrm{m}}(\mathbf{x}) \equiv i^{\mathrm{m}} \frac{\partial^{\mathrm{m}}}{\partial J_{i_{1}} \cdots \partial J_{i_{\mathrm{m}}}}[1+\mathcal{M}(\mathbf{J}, \mathbf{x})]\right|_{\mathbf{J}=0}=\left\langle\left(1+\delta_{1}\right)\left(1+\delta_{2}\right) \Delta u_{i_{1}} \cdots \Delta u_{i_{\mathrm{m}}}\right\rangle,
$$

with $\delta_{1}=\delta\left(\mathbf{x}_{1}\right)$ and $\delta_{2}=\delta\left(\mathbf{x}_{2}\right)$. The PS in the moment expansion approach becomes

$$
(2 \pi)^{3} \delta_{\mathrm{D}}(\mathbf{k})+P_{s}(\mathbf{k})=\sum_{\mathrm{m}=0}^{\infty} \frac{(-i)^{\mathrm{m}}}{\mathrm{m} !} k_{i_{1}} \ldots k_{i_{\mathrm{m}}} \tilde{\Xi}_{i_{1} \cdots i_{\mathrm{m}}}^{\mathrm{m}}(\mathbf{k}),
$$

where the $\tilde{\Xi}_{i_{1} \cdots i_{\mathrm{m}}}^{\mathrm{m}}(\mathbf{k})$ are the Fourier moments of the generating function - the Fourier transforms of their configuration space counterparts, $\Xi_{i_{1} \cdots i_{n}}^{\mathrm{m}}(\mathbf{x})$. The hope is that by cutting the sum in eq. (4.8) at a finite, low moment (m) yields a good approximation to the PS. Indeed, we see that terms linear in the PS appear only for moments $\mathrm{m}=0,1$, and 2 (higher moments involve correlators of at least three fields), while terms $\mathcal{O}\left(P_{L}^{2}\right)$ show up to $\mathrm{m}=4$. From the fifth moment upwards all terms are at least $\mathcal{O}\left(P_{L}^{4}\right)$. Since in this work we want to compute the moments that appear in the 1-loop PS, it is sufficient to cut the sum in eq. (4.8) at $\mathrm{m}=4$, and search for moments up to $\tilde{\Xi}_{i j k l}^{4}(\mathbf{k})$. This is what we do in the next subsection. We choose to take a slow route to do it in order to isolate the moments depending on their dependence on velocity and density fields. This approach will ease to understand further approximations and to compare to different approaches in the literature. 


\subsection{Computation of moments}

We define the m-th scalar velocity moment of the redshift-space PS as

$$
P^{\mathrm{m}}(k, \mu) \equiv \frac{(-i)^{\mathrm{m}}}{\mathrm{m} !} k_{i_{1}} \cdots k_{i_{\mathrm{m}}} \tilde{\Xi}_{i_{1} \cdots i_{\mathrm{m}}}^{\mathrm{m}}(\mathbf{k})=\sum_{n=0}^{\mathrm{m}} \mu^{2 n} f_{0}^{\mathrm{m}} I_{n}^{\mathrm{m}}(k),
$$

such that the total PS is

$$
P_{s}(k, \mu)=\sum_{\mathrm{m}=0}^{\infty} P^{\mathrm{m}}(k, \mu),
$$

up to a Dirac delta function localized at $\mathbf{k}=0$. Whereas the first equality (definition) in eq. (4.9) depends on $\mathbf{k}$, implicitly on $k$ and $\mu \equiv \hat{\mathbf{k}} \cdot \hat{\mathbf{n}}$, the second equality is an ansatz with explicit angular dependence as even powers of $\mu$. This ansatz has been shown to work, at least for moments $\mathrm{m}=0, \ldots, 4$, in Ref. [94]. The functions $I_{n}^{\mathrm{m}}(k)$ will be key to our approach since they encode in a compact way the different expansions entering in the power spectra.

Following, we compute the required moments in which we leave some of the long computations to Appendix A, where we write the $I_{n}^{\mathrm{m}}(k)$ as 2-dimensional integrals.

- The moment $\mathrm{m}=0$ is simply the 2-point real space correlation function

$$
\Xi^{0}(\mathbf{x})=\left\langle\left(1+\delta_{1}\right)\left(1+\delta_{2}\right)\right\rangle=1+\xi(\mathbf{x}) .
$$

In Fourier space $\tilde{\Xi}^{0}(\mathbf{k})=(2 \pi)^{3} \delta_{\mathrm{D}}(\mathbf{k})+P_{\delta \delta}(k)$, or

$$
P^{\mathrm{m}=0}(k, \mu)=P_{\delta \delta}(k),
$$

plus a Dirac delta function localized at $\mathbf{k}=0$, which in the following we will omit. Hence $I_{0}^{0}(k)=P_{\delta \delta}(k)$ is the real space, full nonlinear PS.

- The $\mathrm{m}=1$ moment is

$$
\begin{aligned}
\Xi_{i}^{1}(\mathbf{x}) & =\left\langle\left(1+\delta_{1}\right)\left(1+\delta_{2}\right) \Delta u_{i}\right\rangle=\left\langle\Delta u_{i}\left(\delta_{1}+\delta_{2}\right)\right\rangle+\left\langle\Delta u_{i} \delta_{1} \delta_{2}\right\rangle \\
& \equiv \Xi_{i}^{1, u d}(\mathbf{x})+\Xi_{i}^{1, u d d}(\mathbf{x})
\end{aligned}
$$

where the moment with the label " $u d$ " refers to the correlator constructed by the product of one velocity $(u)$ field and one density $(d)$ field, and " $u d d$ " refers to the correlator containing one velocity and two density fields.

To show how the computations are performed, and for the only time in this work, we work in the detail one of these correlators

$$
\begin{aligned}
& \left\langle\delta\left(\mathbf{x}_{1}\right) \Delta u_{i}\right\rangle=\int \frac{d^{3} k_{1}}{(2 \pi)^{3}} \frac{d^{3} k_{2}}{(2 \pi)^{3}} e^{i \mathbf{k}_{1} \cdot \mathbf{x}_{1}}\left(e^{i \mathbf{k}_{2} \cdot \mathbf{x}_{2}}-e^{i \mathbf{k}_{2} \cdot \mathbf{x}_{1}}\right)\left(i f_{0} \frac{\mathbf{k}_{2} \cdot \hat{\mathbf{n}}}{k_{2}^{2}} \hat{n}_{i}\right)\left\langle\delta\left(\mathbf{k}_{1}\right) \theta\left(\mathbf{k}_{2}\right)\right\rangle \\
& \quad=\int \frac{d^{3} k_{1}}{(2 \pi)^{3}} \frac{d^{3} k_{2}}{(2 \pi)^{3}} e^{i \mathbf{k}_{1} \cdot \mathbf{x}_{1}}\left(e^{i \mathbf{k}_{2} \cdot \mathbf{x}_{2}}-e^{i \mathbf{k}_{2} \cdot \mathbf{x}_{1}}\right)\left(i f_{0} \frac{\mathbf{k}_{2} \cdot \hat{\mathbf{n}}}{k_{2}^{2}} \hat{n}_{i}\right)(2 \pi)^{3} \delta_{\mathrm{D}}\left(\mathbf{k}_{1}+\mathbf{k}_{2}\right) P_{\delta \theta}\left(k_{1}\right) \\
& \quad=-i f_{0} \hat{n}_{i} \int \frac{d^{3} k_{1}}{(2 \pi)^{3}}\left(e^{-i \mathbf{k}_{1} \cdot \mathbf{x}}-1\right) \frac{\mathbf{k}_{1} \cdot \hat{\mathbf{n}}}{k_{1}^{2}} P_{\delta \theta}\left(k_{1}\right)=i f_{0} \hat{n}_{i} \int_{\mathbf{p}} e^{i \mathbf{p} \cdot \mathbf{x}} \frac{\mathbf{p} \cdot \hat{\mathbf{n}}}{p^{2}} P_{\delta \theta}(p),
\end{aligned}
$$

where we used eq. (4.3) to write

$$
\Delta u_{i} \equiv u_{i}\left(\mathbf{x}_{2}\right)-u_{i}\left(\mathbf{x}_{1}\right)=\int_{\mathbf{p}}\left(e^{i \mathbf{p} \cdot \mathbf{x}_{2}}-e^{i \mathbf{p} \cdot \mathbf{x}_{1}}\right)\left(i f_{0} \frac{\mathbf{p} \cdot \hat{\mathbf{n}}}{p^{2}} \hat{n}_{i}\right) \theta(\mathbf{p}) .
$$


Hence, in Fourier space,

$$
\tilde{\Xi}_{i}^{1, u d}(\mathbf{k})=2 i f_{0} \hat{n}_{i} \frac{\mu}{k} P_{\delta \theta}(k) .
$$

Analogously, we compute for the other first order moment,

$$
\tilde{\Xi}_{i}^{1, u d d}(\mathbf{k})=i \hat{n}_{i} f_{0} \int_{\mathbf{p}} \frac{\mathbf{p} \cdot \hat{\mathbf{n}}}{p^{2}}\left[B_{\theta \delta \delta}(\mathbf{p},-\mathbf{k}, \mathbf{k}-\mathbf{p})-B_{\theta \delta \delta}(\mathbf{p},-\mathbf{k}-\mathbf{p}, \mathbf{k})\right]
$$

where the cross bispectrum is

$$
(2 \pi)^{3} \delta_{\mathrm{D}}\left(\mathbf{k}_{1}+\mathbf{k}_{2}+\mathbf{k}_{3}\right) B_{\theta \delta \delta}\left(\mathbf{k}_{1}, \mathbf{k}_{2}, \mathbf{k}_{3}\right)=\left\langle\theta\left(\mathbf{k}_{1}\right) \delta\left(\mathbf{k}_{2}\right) \delta\left(\mathbf{k}_{3}\right)\right\rangle .
$$

Summing the two contributions (4.16) and (4.17) and contracting with $-i k_{i}$ we obtain $P^{\mathrm{m}=1}(\mathbf{k})=-i k_{i} \tilde{\Xi}_{i}^{1}(\mathbf{k})$,

$$
\begin{aligned}
P^{\mathrm{m}=1}(k, \mu) & =2 \mu^{2} f_{0} P_{\delta \theta}(k)+2 k \mu f_{0} \int_{\mathbf{p}} \frac{\mathbf{p} \cdot \hat{\mathbf{n}}}{p^{2}} B_{\theta \delta \delta}(\mathbf{p},-\mathbf{k}, \mathbf{k}-\mathbf{p}) \\
& =\mu^{2} f_{0} I_{1}^{1}(k)
\end{aligned}
$$

with $I_{1}^{1}(k)=I_{1}^{1, u d}(k)+I_{1}^{1, u d d}(k)$, where $I_{1}^{1, u d}(k)=2 P_{\delta \theta}(k)$ and $I_{1}^{1, u d d}(k)$ given in eq. (A.10).

- The second moment, $\mathrm{m}=2$, is

$$
\begin{aligned}
\Xi_{i j}^{2}(\mathbf{x}) & =\left\langle\left(1+\delta_{1}\right)\left(1+\delta_{2}\right) \Delta u_{i} \Delta u_{j}\right\rangle=\left\langle\Delta u_{i} \Delta u_{j}\right\rangle+\left\langle\Delta u_{i} \Delta u_{j}\left(\delta_{1}+\delta_{2}\right)\right\rangle+\left\langle\Delta u_{i} \Delta u_{j} \delta_{1} \delta_{2}\right\rangle \\
& =\Xi_{i j}^{2, u u}(\mathbf{x})+\Xi_{i j}^{2, u u d}(\mathbf{x})+\Xi_{i j}^{2, u u d d}(\mathbf{x}) .
\end{aligned}
$$

Working them out based on their fields dependence, we obtain

$$
\begin{gathered}
\tilde{\Xi}_{i j}^{2, u u}(\mathbf{k})=-2 f_{0}^{2} \hat{n}_{i} \hat{n}_{j} \frac{\mu^{2}}{k^{2}} P_{\theta \theta}(k), \\
\tilde{\Xi}_{i j}^{2, u u d}(\mathbf{k})=-2 f_{0} \hat{n}_{i} \hat{n}_{j} \int_{\mathbf{p}} \frac{\mathbf{p} \cdot \hat{\mathbf{n}}}{p^{2}} \frac{(\mathbf{k}-\mathbf{p}) \cdot \hat{\mathbf{n}}}{|\mathbf{k}-\mathbf{p}|^{2}} B_{\theta \theta \delta}(\mathbf{p}, \mathbf{k}-\mathbf{p},-\mathbf{k}) \\
+2 f_{0} \hat{n}_{i} \hat{n}_{j} \int_{\mathbf{p}} \frac{\mathbf{p} \cdot \hat{\mathbf{n}}}{p^{2}} \frac{\mathbf{k} \cdot \hat{\mathbf{n}}}{k^{2}}\left[B_{\theta \theta \delta}(\mathbf{p}, \mathbf{k},-\mathbf{k}-\mathbf{p})-B_{\theta \theta \delta}(\mathbf{p},-\mathbf{k}, \mathbf{k}-\mathbf{p})\right],
\end{gathered}
$$

and, to 1-loop corrections,

$$
\begin{aligned}
\tilde{\Xi}_{i j}^{2, u u d d}(\mathbf{k})= & -2 f_{0}^{2} \hat{n}_{i} \hat{n}_{j} \int_{\mathbf{p}} \frac{(\mathbf{p} \cdot \hat{\mathbf{n}})^{2}}{p^{4}} P_{\theta \theta}(p)\left[P_{\delta \delta}(|\mathbf{k}-\mathbf{p}|)-P_{\delta \delta}(k)\right] \\
& -2 f_{0}^{2} \hat{n}_{i} \hat{n}_{j} \int_{\mathbf{p}} \frac{\mathbf{p} \cdot \hat{\mathbf{n}}}{p^{2}} \frac{(\mathbf{k}-\mathbf{p}) \cdot \hat{\mathbf{n}}}{|\mathbf{k}-\mathbf{p}|^{2}} P_{\delta \theta}(p) P_{\delta \theta}(|\mathbf{k}-\mathbf{p}|) .
\end{aligned}
$$


Summing up the three contributions, contracting with $-\frac{1}{2} k_{i} k_{j}$, and rearranging terms we have

$$
\begin{aligned}
P^{\mathrm{m}=2}(\mathbf{k})= & f_{0}^{2} \mu^{4} P_{\theta \theta}(k) \\
+ & k \mu f_{0} \int \frac{d^{3} p}{(2 \pi)^{3}} \frac{\mathbf{p} \cdot \hat{\mathbf{n}}}{p^{2}}\left[f_{0} \frac{[(\mathbf{k}-\mathbf{p}) \cdot \hat{\mathbf{n}}]^{2}}{|\mathbf{k}-\mathbf{p}|^{2}} B_{\theta \delta \theta}(\mathbf{p},-\mathbf{k}, \mathbf{k}-\mathbf{p})\right. \\
& \left.\quad-f_{0} \frac{[(\mathbf{k}+\mathbf{p}) \cdot \hat{\mathbf{n}}]^{2}}{|\mathbf{k}+\mathbf{p}|^{2}} B_{\theta \delta \theta}(\mathbf{p}, \mathbf{k},-\mathbf{k}-\mathbf{p})\right] \\
+ & k \mu f_{0} \int \frac{d^{3} p}{(2 \pi)^{3}} \frac{\mathbf{p} \cdot \hat{\mathbf{n}}}{p^{2}}\left[f_{0} \frac{(\mathbf{k} \cdot \hat{\mathbf{n}})^{2}}{k^{2}} B_{\theta \theta \delta}(\mathbf{p},-\mathbf{k}, \mathbf{k}-\mathbf{p})\right. \\
& \left.\quad-f_{0} \frac{(\mathbf{k} \cdot \hat{\mathbf{n}})^{2}}{k^{2}} B_{\theta \theta \delta}(\mathbf{p}, \mathbf{k},-\mathbf{k}-\mathbf{p})\right] \\
+ & \left(k \mu f_{0}\right)^{2} \int \frac{d^{3} p}{(2 \pi)^{3}} \frac{(\mathbf{p} \cdot \hat{\mathbf{n}})^{2}}{p^{4}} P_{\theta \theta}(p)\left[P_{\delta \delta}(|\mathbf{k}-\mathbf{p}|)-P_{\delta \delta}(k)\right] \\
+ & \left(k \mu f_{0}\right)^{2} \int \frac{d^{3} p}{(2 \pi)^{3}} \frac{\mathbf{p} \cdot \hat{\mathbf{n}}}{p^{2}} \frac{(\mathbf{k}-\mathbf{p}) \cdot \hat{\mathbf{n}}}{|\mathbf{k}-\mathbf{p}|^{2}} P_{\delta \theta}(p) P_{\delta \theta}(|\mathbf{k}-\mathbf{p}|),
\end{aligned}
$$

which can be written as

$$
P^{\mathrm{m}=2}(k, \mu)=f_{0}^{2}\left[\mu^{2} I_{1}^{2}(k)+\mu^{4} I_{2}^{2}(k)\right]
$$

with

$$
\begin{aligned}
& I_{1}^{2}(k)=I_{1}^{2, u u}(k)+I_{1}^{2, u u d}(k)+I_{1}^{2, \text { uudd }}(k), \\
& I_{2}^{2}(k)=I_{2}^{2, \text { uu }}(k)+I_{2}^{2, \text { uud }}(k)+I_{2}^{2, \text { uudd }}(k),
\end{aligned}
$$

with $I_{1}^{2, u u}(k)=0, I_{2}^{2, u u}(k)=P_{\theta \theta}(k)$, and the rest of the $I_{1,2}^{2}(k)$ functions are given by eqs. (A.11) and (A.27).

- The third velocity moment, $\mathrm{m}=3$, is

$$
\begin{aligned}
\Xi_{i j k}^{3}(\mathbf{x}) & =\left\langle\left(1+\delta_{1}\right)\left(1+\delta_{2}\right) \Delta u_{i} \Delta u_{j} \Delta u_{k}\right\rangle=\left\langle\Delta u_{i} \Delta u_{j} \Delta u_{k}\right\rangle+\left\langle\left(\delta_{1}+\delta_{2}\right) \Delta u_{i} \Delta u_{j} \Delta u_{k}\right\rangle \\
& =\Xi_{i j k}^{3, \text { uuu }}(\mathbf{x})+\Xi_{i j k}^{3, \text { uuиd }}(\mathbf{x}),
\end{aligned}
$$

where we have not written the term $\left\langle\delta_{1} \delta_{2} \Delta u_{i} \Delta u_{j} \Delta u_{k}\right\rangle$ since it is order $\mathcal{O}\left(P_{L}^{3}\right)$, and not considered here: contrary to the previous moments, whose expressions are valid to arbitrary PT order, in the following we keep only terms up to $\mathcal{O}\left(P_{L}^{2}\right)$. The "uuu" correlator yields

$$
\begin{aligned}
& \tilde{\Xi}_{i j k}^{3, \text { uиu }}(\mathbf{k})=\int d^{3} x e^{-i \mathbf{k} \cdot \mathbf{x}}\left\langle\Delta u_{i} \Delta u_{j} \Delta u_{k}\right\rangle=3 i f_{0}^{3} \hat{n}_{i} \hat{n}_{j} \hat{n}_{k} \int_{\mathbf{p}} \frac{\mathbf{p} \cdot \hat{\mathbf{n}} \mathbf{k} \cdot \hat{\mathbf{n}}}{p^{2}} \\
& \times\left[\frac{(\mathbf{k}+\mathbf{p}) \cdot \hat{\mathbf{n}}}{|\mathbf{k}+\mathbf{p}|^{2}} B_{\theta \theta \theta}(\mathbf{p}, \mathbf{k},-\mathbf{k}-\mathbf{p})-\frac{(\mathbf{k}-\mathbf{p}) \cdot \hat{\mathbf{n}}}{|\mathbf{k}-\mathbf{p}|^{2}} B_{\theta \theta \theta}(\mathbf{p},-\mathbf{k}, \mathbf{k}-\mathbf{p})\right],
\end{aligned}
$$


and the "uuud" term,

$$
\begin{aligned}
\tilde{\Xi}_{i j k}^{3, \text { uud }}(\mathbf{k})= & 12 i f_{0}^{3} \hat{n}_{i} \hat{n}_{j} \hat{n}_{k} \int_{\mathbf{p}} \frac{(\mathbf{p} \cdot \hat{\mathbf{n}})^{2}}{p^{4}} \frac{\mathbf{k} \cdot \hat{\mathbf{n}}}{k^{2}} P_{\theta \theta}(p) P_{\delta \theta}(k) \\
& -12 i f_{0}^{3} \hat{n}_{i} \hat{n}_{j} \hat{n}_{k} \int_{\mathbf{p}} \frac{(\mathbf{p} \cdot \hat{\mathbf{n}})^{2}}{p^{4}} \frac{(\mathbf{k}-\mathbf{p}) \cdot \hat{\mathbf{n}}}{|\mathbf{k}-\mathbf{p}|^{2}} P_{\theta \theta}(p) P_{\delta \theta}(|\mathbf{k}-\mathbf{p}|) .
\end{aligned}
$$

Summing up the two contributions, rearranging some terms and contracting with $\frac{i}{6} k_{i} k_{j} k_{k}$ we have

$$
\begin{aligned}
P^{\mathrm{m}=3}(\mathbf{k})= & -2 k^{2} \mu^{4} f_{0}^{3} \sigma_{v}^{2} P_{\delta \theta}(\mathbf{k}) \\
& +2 k \mu^{3} f_{0}^{3} \int_{\mathbf{p}} \frac{\mathbf{p} \cdot \hat{\mathbf{n}}}{p^{2}} \frac{((\mathbf{k}-\mathbf{p}) \cdot \hat{\mathbf{n}})^{2}}{|\mathbf{k}-\mathbf{p}|^{2}} B_{\theta \theta \theta}(\mathbf{p},-\mathbf{k}, \mathbf{k}-\mathbf{p}) \\
& +2 k^{2} \mu^{2} f_{0}^{3} \int_{\mathbf{p}} \frac{(\mathbf{p} \cdot \hat{\mathbf{n}})^{2}}{p^{4}} \frac{[(\mathbf{k}-\mathbf{p}) \cdot \hat{\mathbf{n}}]^{2}}{|\mathbf{k}-\mathbf{p}|^{2}} P_{\theta \theta}(p) P_{\delta \theta}(|\mathbf{k}-\mathbf{p}|) \\
& +2 k^{2} \mu^{2} f_{0}^{3} \int_{\mathbf{p}} \frac{\mathbf{p} \cdot \hat{\mathbf{n}}}{p^{2}} \frac{[(\mathbf{k}-\mathbf{p}) \cdot \hat{\mathbf{n}}]^{3}}{|\mathbf{k}-\mathbf{p}|^{4}} P_{\delta \theta}(p) P_{\theta \theta}(|\mathbf{k}-\mathbf{p}|),
\end{aligned}
$$

with the velocity variance

$$
\sigma_{v}^{2}=\frac{1}{6 \pi^{2}} \int_{0}^{\infty} d p P_{\theta \theta}^{L}(p)
$$

This can be written as

$$
P^{\mathrm{m}=3}(k, \mu)=f_{0}^{3}\left[\mu^{2} I_{1}^{3}(k)+\mu^{4} I_{2}^{3}(k)+\mu^{6} I_{3}^{3}(k)\right],
$$

with $I_{1}^{3}(k)=0, I_{2}^{3}(k)=I_{2}^{3, \text { uuu }}(k)+I_{2}^{3, \text { uuud }}(k), I_{3}^{3}(k)=I_{3}^{3, \text { uuu }}(k)+I_{3}^{3, \text { uuud }}(k)$, where these $I_{n}^{\mathrm{m}}$ functions are giving by eqs. (A.12) and (A.28).

- Up to 1-loop, the fourth moment is $\Xi_{i j k l}^{\mathrm{m}=4}(\mathbf{x})=\left\langle\Delta u_{i} \Delta u_{j} \Delta u_{k} \Delta u_{l}\right\rangle$, yielding

$$
\begin{aligned}
\tilde{\Xi}_{i j k l}^{4, \text { uuuu }}(\mathbf{k})= & -24 \hat{n}_{i} \hat{n}_{j} \hat{n}_{k} \hat{n}_{l} f_{0}^{4} \int_{\mathbf{p}} \frac{(\mathbf{p} \cdot \hat{\mathbf{n}})^{2}}{p^{4}} \frac{(\mathbf{k} \cdot \hat{\mathbf{n}})^{2}}{k^{4}} P_{\theta \theta}(p) P_{\theta \theta}(k) \\
& +12 \hat{n}_{i} \hat{n}_{j} \hat{n}_{k} \hat{n}_{l} f_{0}^{4} \int_{\mathbf{p}} \frac{(\mathbf{p} \cdot \hat{\mathbf{n}})^{2}}{p^{4}} \frac{((\mathbf{k}-\mathbf{p}) \cdot \hat{\mathbf{n}})^{2}}{|\mathbf{k}-\mathbf{p}|^{4}} P_{\theta \theta}(p) P_{\theta \theta}(|\mathbf{k}-\mathbf{p}|) .
\end{aligned}
$$

After some manipulations we have ${ }^{3}$

$$
\begin{aligned}
P^{\mathrm{m}=4}(\mathbf{k}) & =-k^{2} \mu^{6} f_{0}^{4} \sigma_{v}^{2} P_{\theta \theta}(k) \\
& +\mu^{2} k^{2} f_{0}^{4} \int_{\mathbf{p}} \frac{(\mathbf{p} \cdot \hat{\mathbf{n}})^{3}}{p^{4}} \frac{((\mathbf{k}-\mathbf{p}) \cdot \hat{\mathbf{n}})^{3}}{|\mathbf{k}-\mathbf{p}|^{4}} P_{\theta \theta}(p) P_{\theta \theta}(|\mathbf{k}-\mathbf{p}|) \\
& +\mu^{2} k^{2} f_{0}^{4} \int_{\mathbf{p}} \frac{(\mathbf{p} \cdot \hat{\mathbf{n}})^{2}}{p^{4}} \frac{((\mathbf{k}-\mathbf{p}) \cdot \hat{\mathbf{n}})^{4}}{|\mathbf{k}-\mathbf{p}|^{4}} P_{\theta \theta}(p) P_{\theta \theta}(|\mathbf{k}-\mathbf{p}|) .
\end{aligned}
$$

\footnotetext{
${ }^{3}$ By contracting eq. (4.33) with $\frac{1}{4 !} k_{i} k_{j} k_{k} k_{l}$, the second term carries a factor $(k \mu)^{4}$. Thereafter, we decompose two of these four powers of $k \mu$ as $(k \mu)^{2}=((\mathbf{k}-\mathbf{p}) \cdot \hat{\mathbf{n}})^{2}+2((\mathbf{k}-\mathbf{p}) \cdot \hat{\mathbf{n}})(\mathbf{p} \cdot \hat{\mathbf{n}})+(\mathbf{p} \cdot \hat{\mathbf{n}})^{2}$.
} 

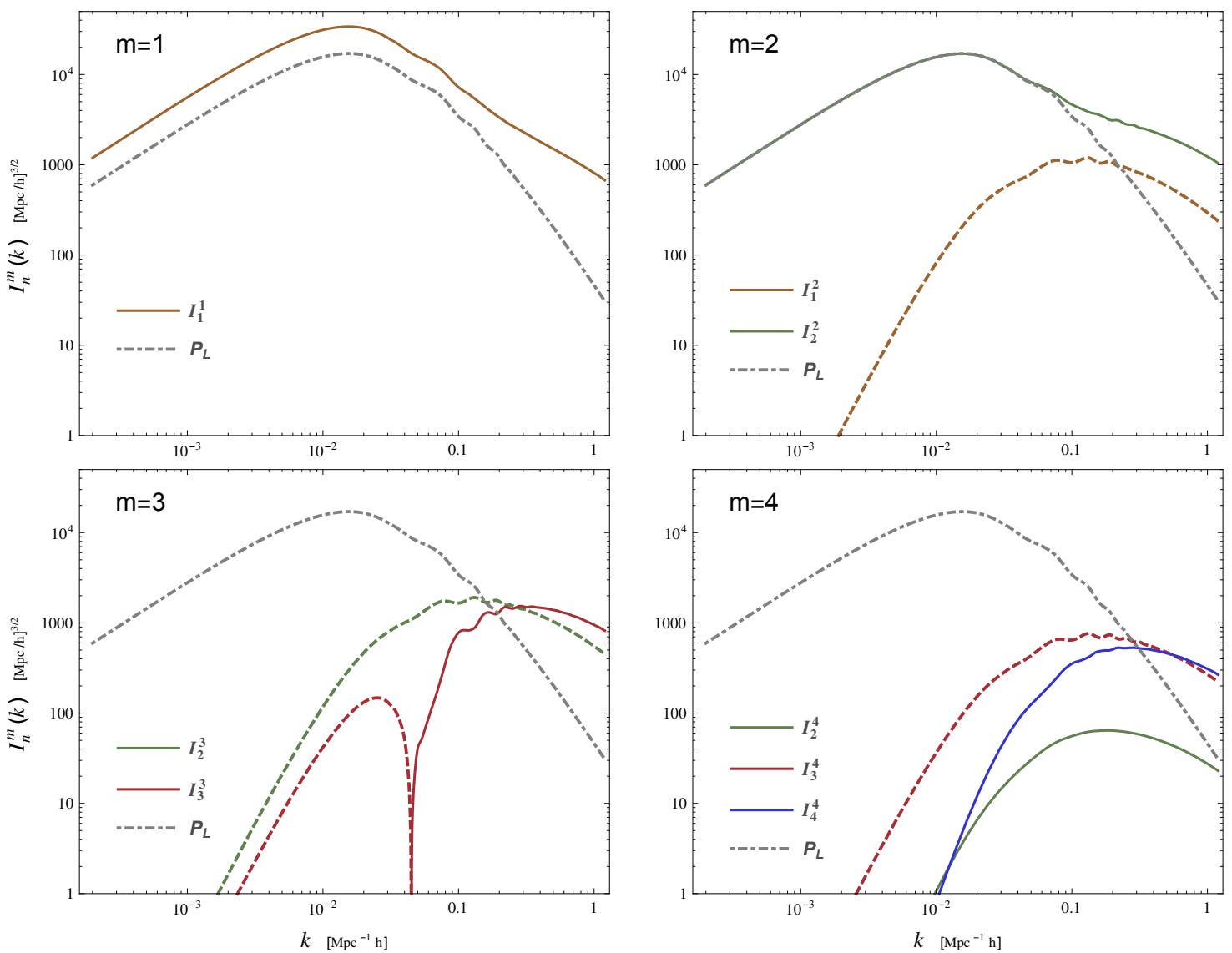

Figure 4. Functions $I_{n}^{\mathrm{m}}(k)$ for dark matter that contribute to the velocity moments through eq. (4.9). We are using F6 MG model at redshift $z=0.5$. Solid lines are for moments, and dashed lines for their negatives. Each panel shows the different $\mathrm{m}$ pieces in which brown lines are for $n=1$, green for $n=2$, red for $n=3$, and blue for $n=4$. For comparison in all panels we also plot the linear matter power spectrum $P_{L}(k)$ which is the linear piece of $I_{0}^{0}(k)$.

This can be written as

$$
P^{\mathrm{m}=4}(k, \mu)=f_{0}^{3}\left[\mu^{2} I_{1}^{4}(k)+\mu^{4} I_{2}^{4}(k)+\mu^{6} I_{3}^{4}(k)+\mu^{8} I_{4}^{4}(k)\right]
$$

with $I_{1}^{4}(k)=0, I_{2}^{4}(k)=I_{2}^{4, \text { uuuu }}(k), I_{3}^{4}(k)=I_{4}^{4, \text { uuuu }}(k)$ and $I_{4}^{4}(k)=I_{4}^{4, \text { uuuu }}(k)$, where these functions are giving by eq. (A.29).

To get a sense of the significance of the different contributions $I_{n}^{\mathrm{m}}(k)$, in figure 4 we plot the non-vanishing of these functions with $\mathrm{m}=1,2,3,4$, for dark matter particles $(b=1$, and all other bias parameters equal to zero); the function $I_{0}^{0}(k)$ is simply given by the densitydensity PS. We employ the F6 model and evaluated results at $z=0.5$. The larger contributions are given by $I_{1}^{1}$ and $I_{2}^{2}$, since these are the only $I$-functions that contain terms linear in the PS, while the rest of the functions yield pure 1-loop contributions. For comparison, on each panel we also show the linear density-density PS $P_{L}(k)$. 


\subsection{Moment expansion approach}

We have split the velocity moments as

$$
\begin{aligned}
\Xi^{0} & =\Xi^{0, d d}, \\
\Xi_{i}^{1} & =\Xi_{i}^{1, u d}+\Xi_{i}^{1, u d d}, \\
\Xi_{i j}^{2} & =\Xi_{i j}^{2, u u}+\Xi_{i j}^{2, \text { uud }}+\Xi_{i j}^{2, u u d d}, \\
\Xi_{i j k}^{3} & =\Xi_{i j k}^{3, \text { uuu }}+\Xi_{i j k}^{3, \text { uuud }}, \\
\Xi_{i j k l}^{4} & =\Xi_{i j k l}^{4, \text { uuuu } .}
\end{aligned}
$$

Now, it should become clear how this splitting is useful to compare models in the literature. To linear order, only the pieces $\tilde{\Xi}^{0, d d}(\mathbf{k}), \tilde{\Xi}_{i}^{1, u d}(\mathbf{k})$, and $\tilde{\Xi}_{i j}^{2, u u}(\mathbf{k})$ contribute yielding [9, 19]:

$$
\begin{aligned}
P_{s}^{K}(k, \mu) & =P_{\delta \delta}^{L}(k)+2 f_{0} \mu^{2} P_{\delta \theta}^{L}(k)+f_{0}^{2} \mu^{4} P_{\theta \theta}^{L}(k), \\
& =b_{1}^{2}\left(1+\mu^{2} \frac{f(k)}{b_{1}}\right)^{2} P_{L}(k)
\end{aligned}
$$

where in the second equality we have omitted $\nabla^{2} \delta_{m}$ biasing to show how the Kaiser formula is recovered, although the Kaiser boost becomes scale dependent due to the growth rate $f(k)$. We generalize, for future use, to the nonlinear Kaiser

$$
\begin{aligned}
P_{s}^{K, \mathrm{NL}}(k, \mu) & =\tilde{\Xi}^{0, d d}(\mathbf{k})-i k_{i} \tilde{\Xi}_{i}^{1, u d}(\mathbf{k})-\frac{1}{2} k_{i} k_{j} \tilde{\Xi}_{i j}^{2, u u}(\mathbf{k}) \\
& =P_{\delta \delta}(k)+2 f_{0} \mu^{2} P_{\delta \theta}(k)+f_{0}^{2} \mu^{4} P_{\theta \theta}(k),
\end{aligned}
$$

which adds the non-linear corrections to the power cross-spectra $P_{\delta \delta, \delta \theta, \theta \theta}$, but neglects some of the next to leading perturbative order, 1-loop contributions.

Consider now all the terms that contain bispectra contributions. These are given in eqs. (4.17), (4.22), and (4.28), and add up as ${ }^{4}$

$$
\begin{aligned}
A( & (k, \mu) \equiv-i k_{i} \tilde{\Xi}_{i}^{1, u d d}(\mathbf{k})-\frac{1}{2} k_{i} k_{j} \tilde{\Xi}_{i j}^{2, u u d}(\mathbf{k})+\frac{i}{6} k_{i} k_{j} k_{k} \tilde{\Xi}_{i j k}^{3, u u u}(\mathbf{k}) \\
= & 2 k \mu f_{0} \int_{\mathbf{p}} \frac{\mathbf{p} \cdot \hat{\mathbf{n}}}{p^{2}} B_{\theta \delta \delta}(\mathbf{p},-\mathbf{k}, \mathbf{k}-\mathbf{p}) \\
& +k \mu f_{0} \int_{\mathbf{p}} \frac{\mathbf{p} \cdot \hat{\mathbf{n}}}{p^{2}}\left[f_{0} \frac{[(\mathbf{k}-\mathbf{p}) \cdot \hat{\mathbf{n}}]^{2}}{|\mathbf{k}-\mathbf{p}|^{2}} B_{\theta \delta \theta}(\mathbf{p},-\mathbf{k}, \mathbf{k}-\mathbf{p})-f_{0} \frac{[(\mathbf{k}+\mathbf{p}) \cdot \hat{\mathbf{n}}]^{2}}{|\mathbf{k}+\mathbf{p}|^{2}} B_{\theta \delta \theta}(\mathbf{p}, \mathbf{k},-\mathbf{k}-\mathbf{p})\right] \\
& +k \mu f_{0} \int_{\mathbf{p}} \frac{\mathbf{p} \cdot \hat{\mathbf{n}}}{p^{2}}\left[f_{0} \frac{(\mathbf{k} \cdot \hat{\mathbf{n}})^{2}}{k^{2}} B_{\theta \theta \delta}(\mathbf{p},-\mathbf{k}, \mathbf{k}-\mathbf{p})-f_{0} \frac{(\mathbf{k} \cdot \hat{\mathbf{n}})^{2}}{k^{2}} B_{\theta \theta \delta}(\mathbf{p}, \mathbf{k},-\mathbf{k}-\mathbf{p})\right] \\
& +2 k \mu f_{0} \int_{\mathbf{p}} \frac{\mathbf{p} \cdot \hat{\mathbf{n}}}{p^{2}} f_{0}^{2} \frac{(\mathbf{k} \cdot \hat{\mathbf{n}})^{2}}{k^{2}} \frac{((\mathbf{k}-\mathbf{p}) \cdot \hat{\mathbf{n}})^{2}}{|\mathbf{k}-\mathbf{p}|^{2}} B_{\theta \theta \theta}(\mathbf{p},-\mathbf{k}, \mathbf{k}-\mathbf{p}) .
\end{aligned}
$$

\footnotetext{
${ }^{4} A(k, \mu)$ is of course a different function than $A(k, t)$ of section 2.
} 
Let us define [22]

$$
\begin{aligned}
B_{\sigma}\left(\mathbf{k}_{1}, \mathbf{k}_{2}, \mathbf{k}_{3}\right) \equiv & B_{\theta \delta \delta}\left(\mathbf{k}_{1}, \mathbf{k}_{2}, \mathbf{k}_{3}\right)+f_{0} \frac{\left(\mathbf{k}_{3} \cdot \hat{\mathbf{n}}\right)^{2}}{k_{3}^{2}} B_{\theta \delta \theta}\left(\mathbf{k}_{1}, \mathbf{k}_{2}, \mathbf{k}_{3}\right) \\
& +f_{0} \frac{\left(\mathbf{k}_{2} \cdot \hat{\mathbf{n}}\right)^{2}}{k_{2}^{2}} B_{\theta \theta \delta}\left(\mathbf{k}_{1}, \mathbf{k}_{2}, \mathbf{k}_{3}\right)+f_{0}^{2} \frac{\left(\mathbf{k}_{2} \cdot \hat{\mathbf{n}}\right)^{2}}{k_{2}^{2}} \frac{\left(\mathbf{k}_{3} \cdot \hat{\mathbf{n}}\right)^{2}}{k_{3}^{2}} B_{\theta \theta \theta}\left(\mathbf{k}_{1}, \mathbf{k}_{2}, \mathbf{k}_{3}\right) \\
= & \left\langle\theta\left(\mathbf{k}_{1}\right)\left[\delta\left(\mathbf{k}_{2}\right)+f_{0} \frac{\left(\mathbf{k}_{2} \cdot \hat{\mathbf{n}}\right)^{2}}{k_{2}^{2}} \theta\left(\mathbf{k}_{2}\right)\right]\left[\delta\left(\mathbf{k}_{3}\right)+f_{0} \frac{\left(\mathbf{k}_{3} \cdot \hat{\mathbf{n}}\right)^{2}}{k_{3}^{2}} \theta\left(\mathbf{k}_{3}\right)\right]\right\rangle^{\prime} .
\end{aligned}
$$

Then, noting the symmetries $B_{\sigma}\left(\mathbf{k}_{1}, \mathbf{k}_{2}, \mathbf{k}_{3}\right)=B_{\sigma}\left(\mathbf{k}_{1}, \mathbf{k}_{3}, \mathbf{k}_{2}\right)=B_{\sigma}\left(-\mathbf{k}_{1},-\mathbf{k}_{2},-\mathbf{k}_{3}\right)$, one can write

$$
A(k, \mu)=2 k \mu f_{0} \int_{\mathbf{p}} \frac{\mathbf{p} \cdot \hat{\mathbf{n}}}{p^{2}} B_{\sigma}(\mathbf{p},-\mathbf{k}, \mathbf{k}-\mathbf{p}),
$$

which is one of the corrections to the non-linear Kaiser model introduced for the TNS model in [22].

The rest of the velocity moments are collected as

$$
D(k, \mu) \equiv-\frac{1}{2} k_{i} k_{j} \tilde{\Xi}_{i j}^{2, u u d d}(\mathbf{k})+\frac{i}{3 !} k_{i} k_{j} k_{k} \tilde{\Xi}_{i j k}^{3, \text { uuud }}(\mathbf{k})+\frac{1}{4 !} k_{i} k_{j} k_{k} k_{l} \tilde{\Xi}_{i j k l}^{4, \text { uuuu }}(\mathbf{k}),
$$

hence this function is constructed out of four velocity or density fields, which at 1-loop only contribute as linear fields. Hence the only involved kernel different than EdS is $G_{1}(k)$. We make the splitting

$$
D(k, \mu)=B(k, \mu)+C(k, \mu)-\left(k \mu f_{0} \sigma_{v}\right)^{2} P_{s}^{K}(k, \mu),
$$

with [22]

$$
\begin{aligned}
B(k, \mu) & =\left(k \mu f_{0}\right)^{2} \int_{\mathbf{p}} F(\mathbf{p}) F(\mathbf{k}-\mathbf{p}), \\
F(\mathbf{p}) & =\frac{\mathbf{p} \cdot \hat{\mathbf{n}}}{p^{2}}\left[P_{\delta \theta}(p)+f_{0} \frac{(\mathbf{p} \cdot \hat{\mathbf{n}})^{2}}{p^{2}} P_{\theta \theta}(p)\right],
\end{aligned}
$$

and

$$
C(k, \mu)=\left(k \mu f_{0}\right)^{2} \int_{\mathbf{p}} \frac{(\mathbf{p} \cdot \hat{\mathbf{n}})^{2}}{p^{4}} P_{\theta \theta}(p) P_{s}^{K}\left(|\mathbf{k}-\mathbf{p}|, \mu_{\mathbf{k}-\mathbf{p}}\right),
$$

with $\mu_{\mathbf{k}-\mathbf{p}}$ the angle between $\mathbf{k}-\mathbf{p}$ and the line of sight $\hat{\mathbf{n}}$.

Finally, the redshift-space PS in the moment expansion (ME) approach to 1-loop in SPT is

$$
P_{s}^{\mathrm{ME}}(k, \mu)=\sum_{\mathrm{m}=0}^{4} \frac{(-i)^{\mathrm{m}}}{\mathrm{m} !} k_{i_{1}} \cdots k_{i_{\mathrm{m}}} \Xi_{i_{1} \cdots i_{\mathrm{m}}}^{\mathrm{m}}(\mathbf{k})=P_{s}^{K, \mathrm{NL}}(k, \mu)+A(k, \mu)+D(k, \mu) .
$$

Note that eq. (4.47) is known from [22] (eq. (23) in that paper), but we have used the ME formalism of refs. [19, 72] to derive it, and we have generalized it for tracers in arbitrary cosmologies. However, this is not the TNS RSD model expression, since the authors preferred to consider another expression with a phenomenological FoG damping term (eq. (18) in their paper), instead of the exact expression. In section 4.5 we will use EFT-counterterms to model the missing effects of non-linear mappings between redshift and real space coordinates and FoG features as has been done recently in several works $[73,74,76,95,96]$. 

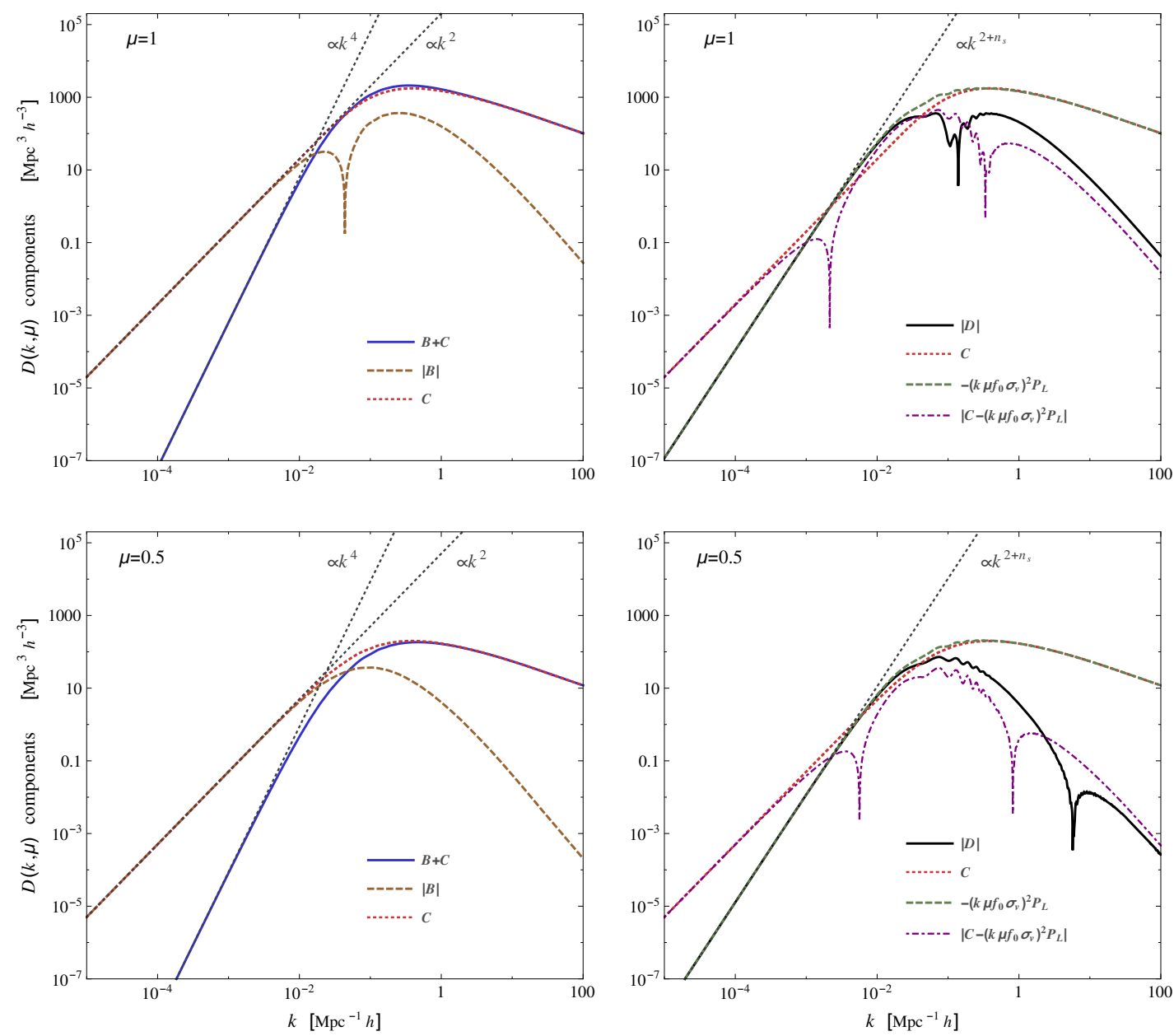

Figure 5. Components of $D(k, \mu)$ according to the splitting of eq. (4.43) for the model F6 at redshift $z=0.5$ and for line-of-sight angle directions $\mu=1$ (top panels) and $\mu=0.5$ (bottom panels). On the left panels we show that $B$ and $C$ functions behave as $k^{2}$ at large scales, but the sum $B+C$ goes as $k^{4}$, while at small scales $B+C \sim C$. On the right panels we show the effects of adding the component $\propto-k^{2} P_{L}(k)$, which at large scales dominates rendering $D(k \rightarrow 0, \mu) \propto k^{2} P_{L}(k) \propto k^{2+n_{s}}$, while at small scales it cancels the dominant contribution to $C$ and makes $D \propto B$.

\subsection{Large and small scales behavior}

We now want to check the IR and UV behavior of the functions composing the ME PS, eq. (4.47). Taking their large-scale limit, up to order $\mathcal{O}\left(k^{2}\right)$ we obtain

$$
B(k \rightarrow 0, \mu)=-C(k \rightarrow 0, \mu)=-\left(k \mu f_{0}\right)^{2} \int_{\mathbf{p}} \mu_{\mathbf{p}}^{2}\left(1+f_{0} \mu_{\mathbf{p}}^{2}\right)^{2} \frac{P_{L}^{2}(p)}{p^{2}} \propto k^{2},
$$

such that UV cancellations provide $B(k \rightarrow 0, \mu)+C(k \rightarrow 0, \mu) \propto k^{4}$. Hence at large scales the dominant term in function $D$ is $-\left(k \mu f_{0} \sigma_{v}\right)^{2} P_{s}^{K}(k, \mu)$ and it behaves as

$$
D(k \rightarrow 0, \mu) \propto k^{2} P_{L}(k) \propto k^{2+n_{s}},
$$

with $n_{s}$ the primordial spectral index. Hence, $B$ grows faster than $k^{2} P_{L}$ at low- $k$, which would violate momentum conservation, yielding that small scales largely affect the loop contributions at large scales. $C$ cancels this pathological behaviour, and $B+C$ goes as $k^{4}$. It is the term $-\sigma_{v}^{2} k^{2} P_{\theta \theta}(k)$ the one that brings the correct behaviour, $D \rightarrow k^{2} P_{L}$ at large scales. 
On the other limit, at high- $k$, we obtain an IR divergence in $C(k, \mu)$ for power spectra with spectral index $n \leq-1$, which is canceled out by the term $-\sigma_{v}^{2} k^{2} P_{\theta \theta}(k)$. Indeed, we can expand for $p \leq k$, assuming a scale invariant PS $P \propto k^{n}$ which is a good approximation for high $k$, so $k^{m} d^{m} P_{L}(k) / d k^{m} \propto P_{L}(k)$, and we have

$$
P_{s}^{K}\left(|\mathbf{k}-\mathbf{p}|, \mu_{\mathbf{k}-\mathbf{p}}\right)=P_{s}^{K}(k, \mu)+P_{L}(k) \sum_{m=1}^{\infty} \alpha_{m}\left(\frac{p}{k}\right)^{m},
$$

where the coefficients $\alpha_{m}$ are constructed out of contractions of up to $m$ angular directions $\hat{p}_{i}$ :

$$
\alpha_{m}=\sum_{i=0}^{m} \tilde{\alpha}_{s}^{j_{1} \cdots j_{i}} \hat{p}_{j_{1}} \cdots \hat{p}_{j_{i}}
$$

such that if $m$ is odd (even) each term of the above sum contains only an odd (even) number of vectors $\hat{p}_{i}$. Hence,

$$
\begin{aligned}
C(k, \mu)- & \left(k \mu f_{0} \sigma_{v}\right)^{2} P_{s}^{K}(k, \mu)= \\
& \left(k \mu f_{0}\right)^{2} \int \frac{d^{3} p}{(2 \pi)^{3}} \frac{(\mathbf{p} \cdot \hat{\mathbf{n}})^{2}}{p^{4}} P_{\theta \theta}(p)\left[P_{s}^{K}\left(|\mathbf{k}-\mathbf{p}|, \mu_{\mathbf{k}-\mathbf{p}}\right)-P_{s}^{K}(k, \mu)\right] \\
& \ni\left(k \mu f_{0}\right)^{2} P_{L}(k) \int_{p \ll k} \frac{d^{3} p}{(2 \pi)^{3}} \frac{(\mathbf{p} \cdot \hat{\mathbf{n}})^{2}}{p^{4}} P_{\theta \theta}(p)\left[\alpha_{2} \frac{p^{2}}{k^{2}}+\mathcal{O}\left(\frac{p^{4}}{k^{4}}\right)\right],
\end{aligned}
$$

where in the last line of the above equation we used that the first term in the expansion of eq. (4.50) factorizes the angular dependence as $\alpha_{1}=\tilde{\alpha}_{i} \hat{p}^{i}$, such that when performing the angular integration in eq. (4.52) we find $\int d \Omega_{\mathrm{p}} \hat{p}_{i} \hat{p}_{j} \hat{p}_{k}=0$, hence this term vanishes and the first correction is of order $p^{2} / k^{2}$; for the same reason all odd powers of $(p / k)$ are not present, and the second correction is $\mathcal{O}\left((p / k)^{4}\right)$. Hence the combination $C(k, \mu)-\left(k \mu f_{0} \sigma_{v}\right)^{2} P_{s}^{K}(k, \mu)$ is IR divergent only for $n \leq-3$, and safe for typical power spectra. This cancellation is equivalent to the real space 1-loop SPT PS for which the function $P_{22}(k)$ has an IR divergence for $n \leq-1$, that is cured by a term $-\sigma_{\Psi}^{2} k^{2} P_{L}(k) \in P_{13}(k)$, with $\sigma_{\Psi}^{2}$ the variance of Lagrangian displacements.

Summarizing, we notice that the full $D(k, \mu)$ is free of UV and IR divergence for typical power spectra. However, the three pieces that compose it in our splitting, eq. (4.43), have to be present, such that if any of them would be missing there would exist either an IR or an UV divergence. To make this point more clear, in figure 5 we plot the different components of the $D(k, \mu)$ function for angles $\mu=0.5$ (bottom panels) and 1 (top panels). At large scales $D(k, \mu) \propto k^{2+n_{s}}$, although both $B(k, \mu)$ and $C(k, \mu)$ behave as $k^{2}$. Meanwhile, at small scales function $B(k, \mu)$ behaves equally to $C(k, \mu)-\left(k \mu f_{0} \sigma_{v}\right)^{2} P_{L}(k)$, both scaling approximately as $k^{1 / 2} P_{L}(k)$.

\subsection{EFT counterterms}

So far, we have followed a standard PT approach to construct the velocity moments and the complete redshift space PS in the SPT-ME approach. However, loop integrals are of the form $I(\mathbf{k})=\int_{\mathbf{p}} K(\mathbf{k}, \mathbf{p})$ and are computed over all internal momentum space, although $K(\mathbf{k}, \mathbf{p})$ does not hold at all scales, particularly for high internal momentum. Though these kernels are typically suppressed for regions $p \gg k$, such that small scales do not affect considerably the $I(k)$ functions at moderate, quasilinear scales, they pose a fundamentally wrong UV behaviour 
-in particular $P_{13}$. The EFT for large scale structure [97, 98] formalism cuts-off the loop integrals, by directly smoothing the overdensity fields by an arbitrary scale, and introduces counterterms necessary to remove the cut-off dependence on the final expressions. This is dramatically more important in correlators of fields that do not vanish at zero separation, as $\sigma_{v}^{2}$. The objective of EFT is to cure the spurious high- $k$ effects on statistics due to non modeled small scale physics, out of the reach of PT. Further, dark matter evolution is dictated by the Boltzmann equation, and its simplified description with momentum conservation and Euler equation breaks down by nonlinear collapse which makes different streams to converge, leading to velocity dispersion, the formation of matter caustics and, ultimately, to shell crossing. Hence, the very concept of CDM as a coherent fluid at all scales with no velocity dispersion is theoretically inconsistent because of gravitational collapse, breaking down at shell-crossing at best, and very rapidly all the Boltzmann hierarchy is necessary to describe the dynamics; this a key concern of EFT. In real space, for the PS, the leading order EFT correction counterterm (ct) is given by $P_{c t}(k)=-c_{s}^{2}(t) k^{2} P_{L}(k)$, with $c_{s}$ the effective speed of sound of dark matter arising from fluid equations of a non-perfect fluid.

In redshift space, the situation is more complex, because the counterterms not only model small scales, non-perturbative physics, but also the non-linear mathematical map between real space and redshift space densities $[73,74,95]$. In this case, each moment $P^{\mathrm{m}}$ carries its own counterterms of the form $\sum \tilde{\alpha}_{n} \mu^{2 n} k^{2} P_{L}(k)$, leading to, see e.g. [73, 74],

$$
P_{c t}(k, \mu)=\left(\alpha_{0}+\alpha_{1} \mu^{2}+\cdots\right) k^{2} P_{L}(k) .
$$

Finally, along the line-of-sight direction, 2-point statistics are dominated by FoG as a nonlinear coupling between the velocity and density fields, with a characteristic scale given by the velocity dispersion $\sigma_{v}$, for which typically $\sigma_{v}^{-1} \sim k_{\mathrm{NL}}$, motivating to go beyond the leading order through the ansatz [74]

$$
P_{c t}^{\mathrm{NLO}}(k, \mu)=\tilde{c}\left(\mu k f_{0} \sigma_{v}\right)^{4} P_{s}^{K}(k, \mu),
$$

which can be also understood as stemming from a Taylor series expansion of a phenomenological damping factor $\mathcal{D}_{\text {FoG }}\left[\left(k \mu f_{0} \sigma_{v}\right)^{2}\right]$ at second order on $\left(k^{2} / \sigma_{v}^{-2}\right)$. But, as discussed above, $\sigma_{v}$ is not well modeled by PT, and historically replaced by a free parameter $\sigma_{\text {FoG }}$. Here, it becomes modeled by the $\tilde{c}$ time-dependent counterterm. The multipoles become

$$
P_{c t, \ell}^{\mathrm{NLO}}(k)=\tilde{c} \mathcal{D}_{c t, \ell}^{\mathrm{NLO}}(k) f_{0}^{4} k^{4} P_{L}(k),
$$

with

$$
\begin{aligned}
& \mathcal{D}_{c t, \ell=0}^{\mathrm{NLO}}(k)=\frac{b_{1}^{2}}{5}+\frac{2}{7} b_{1} f(k)+\frac{f^{2}(k)}{9}, \\
& \mathcal{D}_{c t, \ell=2}^{\mathrm{NLO}}(k)=\frac{4 b_{1}^{2}}{7}+\frac{20}{21} b_{1} f(k)+\frac{40 f^{2}(k)}{99}, \\
& \mathcal{D}_{c t, \ell=4}^{\mathrm{NLO}}(k)=\frac{8 b_{1}^{2}}{35}+\frac{48}{77} b_{1} f(k)+\frac{48 f^{2}(k)}{143} .
\end{aligned}
$$

It is interesting to note that, regardless of the bias and growth rate, the next-to-leading order FoG counterterm contribution to the quadrupole is the largest, about $\mathcal{D}_{c t, \ell=2}^{\mathrm{NLO}} \sim 3 \mathcal{D}_{c t, \ell=0}^{\mathrm{NLO}}$.

We arrive to our final expression for the EFT-Moment expansion approach modeling of the redshift space PS,

$$
P_{s}^{\mathrm{EFT}}(k, \mu)=P_{s}^{\mathrm{ME}}(k, \mu)+\left(\alpha_{0}+\alpha_{1} \mu^{2}+\cdots\right) k^{2} P_{L}(k)+\tilde{c}\left(\mu k f_{0}\right)^{4} P_{s}^{K}(k, \mu)+P_{\text {shot }},
$$




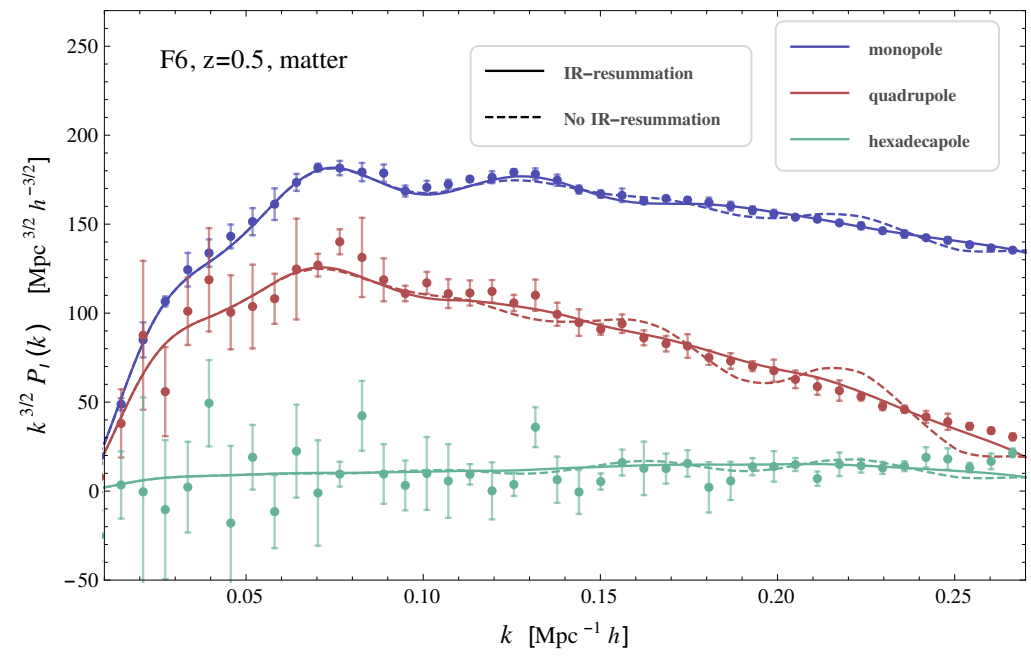

Figure 6. Matter redshift-space power spectrum multipoles with (solid lines) and without (dashed lines) the IR-resummations for MG model F6 at redshift $z=0.5$. The effect of IR-resummation is clearly seen and necessary to degrade the BAO oscillations at high- $k$. The error bars denote the RMS error over the five realizations in the simulations.

with $P_{\text {shot }}$ modeling the stochastic terms, uncorrelated with long wave-length fluctuations. That is, we assume that stochasticity is well localized, with a small range of coherence, such that its spectra can be characterized by a constant (both in $k$ and $\mu$ ) shot noise $P_{\text {shot }}$. In contrast, in e.g. refs. [73, 99-101] the stochastic noise has a component proportional to $(k \mu)^{2}$, leading to two free parameters to model it. Here, we are adopting the most simple prescription where the stochastic contribution is given by a constant shot noise, affecting only the monopole of the PS; see e.g. [74] for a recent use with real data ${ }^{5}$. We will find that this approach yields accurate results when comparing to simulated data.

\subsection{IR-resummation}

Despite the success of SPT-EFT in modeling the broadband PS, the theory yet gives poor results in modeling the BAO since long-wavelength displacement fields, though being essentially linear, stream largely contributing to damp features in the PS in a manner that is non-perturbative under an SPT scheme [103-106]. Then, in order to model the spread and degradation of the $\mathrm{BAO}$ oscillations due to large scale bulk flows, we employ IR-resummations [107-115] as implemented in [74-76]. The main idea is to split the real space linear PS in one piece containing the oscillations (wiggles), $P_{\mathrm{w}}$, and a smooth piece without them, $P_{\mathrm{nw}}$, such that $P_{L}(k)=P_{\mathrm{w}}(k)+P_{\mathrm{nw}}(k)$, to do this we use the method based on fast sine transforms of [116] (proposed in appendix (A.1) of that paper). Note that this approach is somewhat arbitrary, since there is not a single way to tell what is a PS without BAO, so there is a handcrafted element in the process. ${ }^{6}$

\footnotetext{
${ }^{5}$ In contrast, in $[99,102]$ a more cumbersome stochastic expression, including $k^{2}$ and $(\mu k)^{2}$ contributions, is used to fit DR12 BOSS data.

${ }^{6}$ In this sense, the LPT approach is more natural, because the Lagrangian displacements of bulk flows are fully resummed from the beginning (see e.g. [73]). Recently, this scheme has been extended to resum also the velocity fields [117], such that a posteriori IR-resummations are no longer required.
} 
To leading order one gets the Kaiser, IR-resummed PS [75]

$$
P_{s}^{K, \mathrm{IR}}(k, \mu)=\left(b_{1}+f(k) \mu^{2}\right)^{2}\left[e^{-k^{2} \Sigma_{\mathrm{tot}}^{2}(k, \mu)} P_{L}(k)+\left(1-e^{-k^{2} \Sigma_{\mathrm{tot}}^{2}(k, \mu)}\right) P_{\mathrm{nw}}(k)\right]
$$

with damping, angle-dependent, factor

$$
\Sigma_{\text {tot }}^{2}(k, \mu)=\left[1+f(k) \mu^{2}(2+f(k))\right] \Sigma^{2}+f^{2}(k) \mu^{2}\left(\mu^{2}-1\right) \delta \Sigma^{2},
$$

with $\Sigma^{2}$ the real space isotropic damping and $\delta \Sigma^{2}$ a subdominant contribution,

$$
\begin{aligned}
\Sigma^{2} & =\frac{1}{6 \pi^{2}} \int_{0}^{k_{s}} d p P_{\mathrm{nw}}(p)\left[1-j_{0}\left(\frac{p}{k_{\mathrm{BAO}}}\right)+2 j_{2}\left(\frac{p}{k_{\mathrm{BAO}}}\right)\right], \\
\delta \Sigma^{2} & =\frac{1}{2 \pi^{2}} \int_{0}^{k_{s}} d p P_{\mathrm{nw}}(p) j_{2}\left(\frac{p}{k_{\mathrm{BAO}}}\right),
\end{aligned}
$$

where $k_{s}$ is a scale separating long and short modes and $k_{\mathrm{BAO}} \simeq\left(105 \mathrm{Mpc} h^{-1}\right)^{-1}$ corresponds to the BAO scale. Functions $j_{n}$ are the spherical Bessel functions of order $n$. The choice of $k_{s}$ is also arbitrary, but the results depend very weakly on it, as long as $k_{s} \gtrsim 0.05 \mathrm{Mpc}^{-1} h$ [76]; in this work we use $k_{s}=0.2 \mathrm{Mpc}^{-1} h$.

To 1-loop the IR-resummed PS becomes [75]

$$
\begin{aligned}
P_{s}^{\mathrm{IR}}(k, \mu)= & e^{-k^{2} \Sigma_{\text {tot }}^{2}(k, \mu)} P_{s}^{\mathrm{EFT}}(k, \mu)+\left(1-e^{-k^{2} \Sigma_{\text {tot }}^{2}(k, \mu)}\right) P_{\mathrm{s}, \mathrm{nw}}^{\mathrm{EFT}}(k, \mu) \\
& +e^{-k^{2} \Sigma_{\text {tot }}^{2}(k, \mu)} P_{\mathrm{w}}(k) k^{2} \Sigma_{\text {tot }}^{2}(k, \mu),
\end{aligned}
$$

where $P_{s}^{\mathrm{EFT}}(k, \mu)$ is the 1-loop PS computed using eq. (4.59), and $P_{\mathrm{s}, \mathrm{nw}}^{\mathrm{EF}}(k, \mu)$ is also computed with eq. (4.59) but using as input the non-wiggle linear PS $P_{\mathrm{nw}}$. Equation (4.64) is our final expression to be compared with simulated halo statistics in section 5 .

In figure 6 we show the matter redshift-space PS multipoles $(\ell=0,2,4)$ with and without the IR-resummations for MG model F6 at redshift $z=0.5$. We notice that the BAO degradation is not well modeled by eq. (4.59) alone, as large oscillations are still present at high- $k$ values (dashed lines) but it needs the IR-resummation presented in this section to follow more appropriately the matter particle data, as shown in solid lines (the details of the simulations are presented in the next section).

We finally notice that we have used the same resummation scheme as for $\Lambda \mathrm{CDM}$, with the generalized cosmologies effects entering through the scale-dependent growth rate $f(k)$ and the linear PS. This pragmatic approach is natural for generalized cosmologies that reduce to $\Lambda \mathrm{CDM}$ at large scales, since the bulk flows that are treated non-perturbatively are almost indistinguishable among the different models. ${ }^{7}$

\section{$5 \quad$ Numerical Results}

Having laid out our perturbation theory framework in the previous sections, here we proceed to compare our theoretical predictions for the PS from eq. (4.64), against the ones obtained by state-of-the-art $N$-body simulations.

\footnotetext{
${ }^{7} \mathrm{MG}$ theories like DGP [118] and cubic Galileons [119] do not reduce to $\Lambda$ CDM at large scales. However, the linear growth rate $f$ is scale independent in these cases, suggesting this IR-resummation approximation is valid for such theories as well.
} 

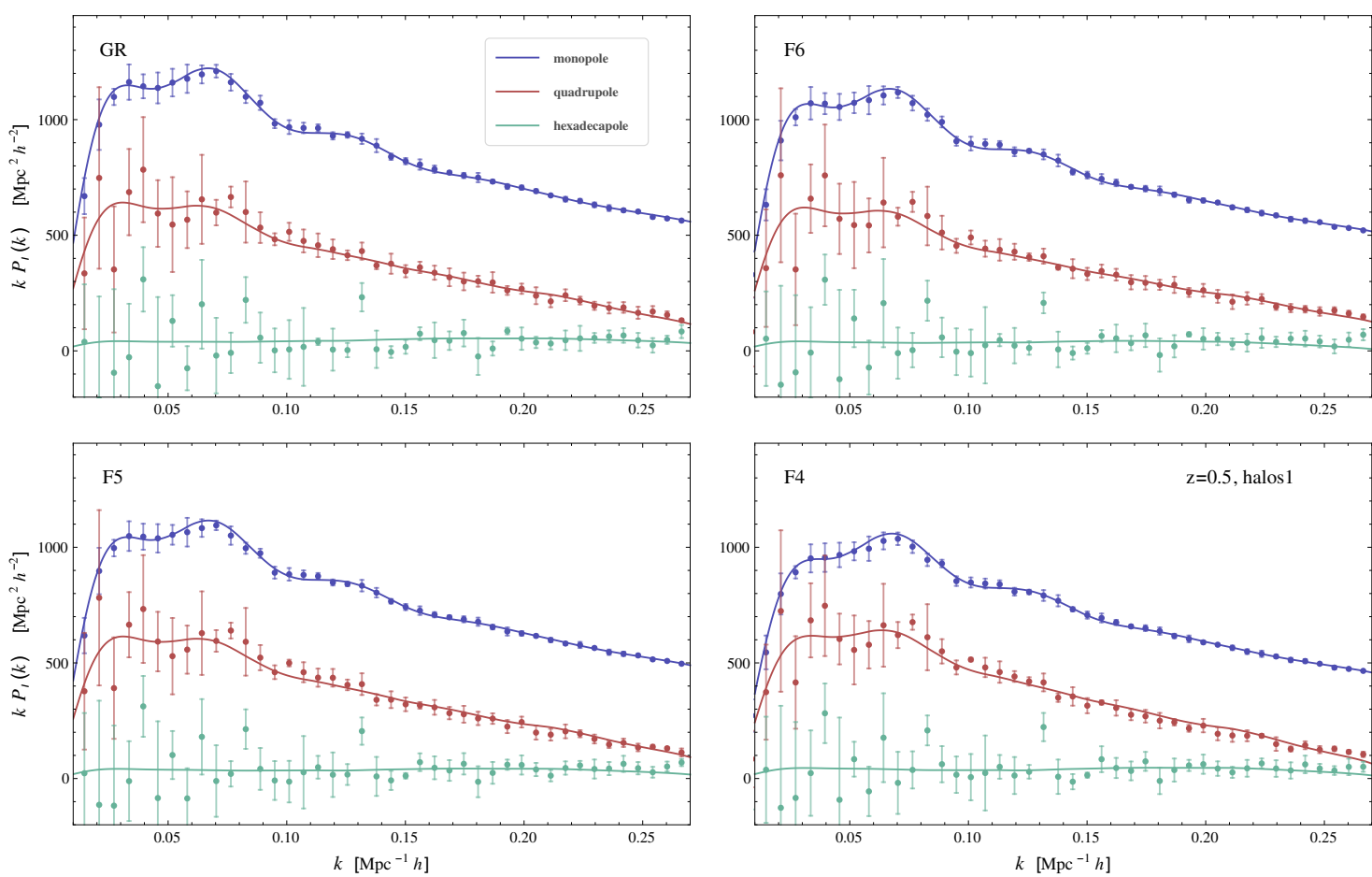

Figure 7. Redshift-space power spectrum monopole, quadrupole, and hexadecapole for halo catalogue $1\left(10^{12}<M_{h}<4.5 \times 10^{12} M_{\odot} h^{-1}\right)$ at redshift $z=0.5$.

Before discussing the results, we first begin with a brief overview of the $N$-body simulations we compare with, which are the Extended LEnsing PHysics using ANalaytic ray Tracing ELEPHANT simulations [36, 79], that were performed with a modified version of the RAMSES code, the ECOSMOG module $[120,121]$. The $\Lambda$ CDM runs correspond to the following set of parameters $\left\{\Omega_{m}, \Omega_{\Lambda}, h, n_{s}, \sigma_{8}, \Omega_{b}\right\}=\{0.281,0.719,0.697,0.971,0.848,0.046\}$, while 3 instances of the HS $n=1 f(R)$ model were simulated, corresponding to three variations of $\left|\bar{f}_{R_{0}}\right|=\left\{10^{-6}, 10^{-5}, 10^{-4}\right\}$. Furthermore, each scenario has been run using 5 different initial random seeds, that we average over. For the purposes of simplicity, we refer to these scenarios as GR, F6, F5, and F4, respectively, and we will also focus on snapshots at $z=0.5$ and $z=1$. The simulations span a cubic volume of $V_{\text {box }}=\left(1024 \mathrm{Mpc} h^{-1}\right)^{3}$, with $1024^{3}$ dark matter particles, while gravitationally bound haloes were identified using the publicly available code ROCKSTAR [122]. Further details about the simulations can be found at [36, 79].

To generate RSD outputs from the simulated dark matter and halo catalogues, we displace the particle (and halo) positions with their peculiar velocities, in accordance with the mapping (4.1), choosing the line of sight $\hat{\mathbf{n}}$ along the $\hat{\mathbf{z}}$ axis. We utilize the publicly available code NbodyKit ${ }^{8}$ in order to extract the first 3 nonvanishing multipoles of the matter and halo PS, $P_{0}(k), P_{2}(k), P_{4}(k)$, evaluated at 512 equally spaced $k$ bins in the range $0.00614<k<3.135 \mathrm{Mpc}^{-1} h$. In the halo case, we extract two separate sub-samples, spanning halo mass ranges of $12<\log _{10} M_{h}<12.65$ and $12.65<\log _{10} M_{h}<13$ in units of $M_{\odot} h^{-1}$, that we will refer to as halo catalogue 1 and 2 , respectively, from now on. Upon

\footnotetext{
${ }^{8}$ https://nbodykit.readthedocs.io/en/latest/index.html
} 

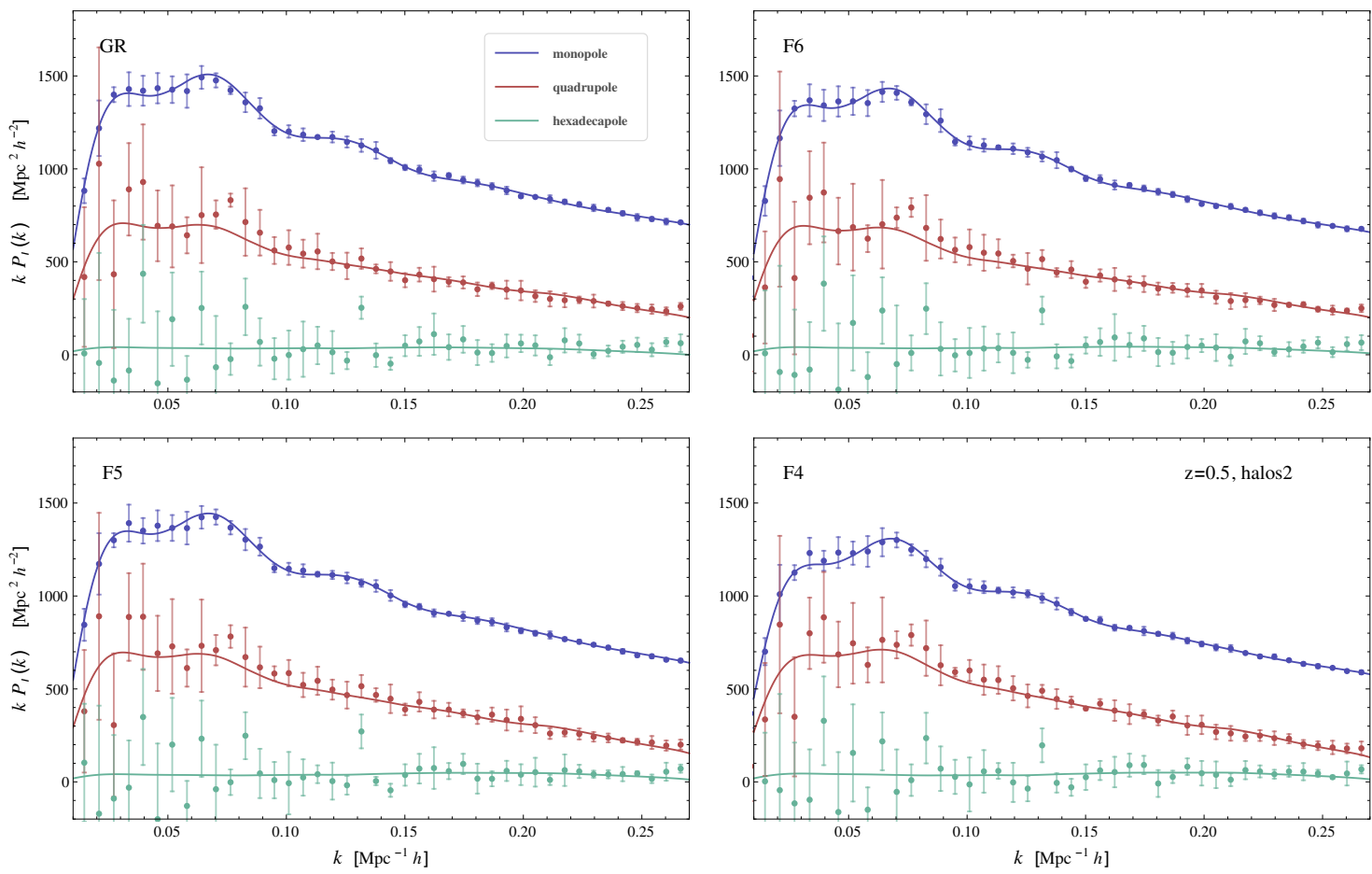

Figure 8. Redshift-space power spectrum multipoles for halo catalogue $2\left(4.5 \times 10^{12}<M_{h}<1 \times 10^{13}\right.$ $\left.M_{\odot} h^{-1}\right)$ at redshift $z=0.5$.

the extraction of the clustering statistics from the halo catalogues, we include both main and satellite haloes in our calculation. We further note that one could alternatively only work with the main gravitationally bound structures identified by the halo finder, as was e.g. done in [51], in which case our model would still be applicable, and the agreement with the simulations would likely be better. Given however that the former scenario is more realistic, we did choose to include the sub-halos and still found good agreement with our model's predictions, as we will see shortly.

The final missing piece in order to compare against the simulations pertains to the determination of the model free parameters, consisting of a total of 4 bias parameters $\left\{b_{1}, b_{2}, b_{s^{2}}, b_{3 n l}\right\}$ and 4 additional EFT parameters $\left\{c_{0}, c_{2}, c_{4}, \tilde{c}\right\}$, with $c_{\ell}$ the multipoles of the factor $\left(\alpha_{0}+\right.$ $\left.\alpha_{2} \mu^{2}+\cdots\right)$ in eq. (4.59). We keep the shot-noise Poissonian, such that $P_{\text {shot }}=1 / \bar{n}$, with $\bar{n}$ the mean number density of halos. Although one has the liberty to let this parameter free, or even adding a dependence on $\mu^{2} k^{2}[73,100,101]$, we find that the Poissonian noise gives accurate results for our simulated data. We will further reduce the number of independent parameters from 8 to 6 , since $b_{s^{2}}$ and $b_{3 n l}$ can be expressed in terms of $b_{1}$, from EdS co-evolution [78], as

$$
\begin{aligned}
b_{s^{2}} & =-\frac{4}{7}\left(b_{1}-1\right), \\
b_{3 n l} & =\frac{32}{315}\left(b_{1}-1\right) .
\end{aligned}
$$

These expressions assume that the initial, Lagrangian bias is only local and that the velocity 
field is unbiased. The above relations were obtained using EdS evolution; however, we find that they yield acceptable results also in the MG models considered here, so we use them to reduce the number of free parameters of the theory. In the case of simpler local bias models, one can analytically predict the bias parameters in MG, as was done e.g. in [83, 123]. This is not the case for the higher order bias and EFT parameters considered in this work, which cannot be modeled from first principles. As a result, we determine these values by fitting our model's predictions (4.64) to the simulated multipoles, through Markov Chain Monte Carlo runs ${ }^{9}$ over the range $0<k<0.25 \mathrm{Mpc}^{-1} h$, and report the best-fit values given by the maximum-likelihood estimators, and $1 \sigma$ confidence intervals for each sample in Table $1 .{ }^{10}$ Finally, we note that the linear PS for the base $\Lambda$ CDM cosmology is obtained by CAMB $^{11}$ [126], and the linear PS of MG models are computed as

$$
P_{L}^{\mathrm{MG}}(k, z)=\left(\frac{D_{+}(k, z)}{D_{+}(k \rightarrow 0, z)}\right)^{2} P_{L}^{\Lambda \mathrm{CDM}}(k, z),
$$

which is an excellent approximation as long as MG effects are negligible at early times for the scales of interest, which is certainly the case for $\left|f_{R 0}\right| \leq 10^{-4}$.

Before we proceed to discuss the results for haloes, we begin by considering the relatively simpler case of dark matter, which is shown in figure 6. There, we compare our theoretical predictions for the monopole, quadrupole and hexadecapole of the matter PS, against the ones from the $N$-body simulations, for the F6 model at $z=0.5$. The prediction for the monopole remains consistent (within $1 \sigma$ error bars) with the simulation down to $k \sim 0.26 \mathrm{Mpc}^{-1} h$, with the higher order multipoles achieving similar levels of accuracy down to wave-modes of $k \sim 0.24 \mathrm{Mpc}^{-1} h$. Even though the larger error bars in the latter case (which are expected given the limited simulation volume and sample variance) do not allow us to perform strict comparisons around the BAO scale, the model predictions are nevertheless well consistent with the simulated trend. We finally add that we checked and found very similar agreement, as in the F6 case, for the rest of the gravity models, that we do not show here. In [50] similar comparisons were performed to HS models using the TNS model, but without the use of EFT parameters and IR-resummation, and hence its level of accuracy is more limited than in this work.

In figures 7 and 8, we compare our model predictions (4.64) for the first three multipoles of the halo PS for all gravity models, against the ones obtained from the simulated halo catalogues 1 and 2, respectively, at $z=0.5$. The error bars show the RMS errors for the five realizations on each model. Starting with the halo 1 sample, we recover very similar levels of accuracy for the F6, F5, and F4 gravity models as in the known GR case, demonstrating a robust behavior across the parameter space of the Hu-Sawicki model. As in the dark matter case of figure 6 , the monopole predictions are the most accurate down to higher $k$ modes, up to $k=0.27 \mathrm{Mpc}^{-1} h$, but the model also performs very well for the higher multipoles, demonstrating consistency with the corresponding simulated curves well within the non-linear regime. The more pronounced error bars, due to cosmic variance, in the higher multipoles are once again expected, especially given the relatively smaller number of halos compared to the

\footnotetext{
${ }^{9}$ We use the generic sampler https://github.com/rodriguezmeza/MathematicaMCMC-1.0.0.git [124].

${ }^{10} \mathrm{We}$ should note, at this point, that during the fitting process we have assumed a diagonal covariance matrix. Even though we know that in principle the errors in different bins are correlated and expect that the inclusion of the non-diagonal terms would tighten the constraints. We nevertheless obtain very good fits, as it was also found in, e.g., [125] that made similar assumptions.

${ }^{11}$ https://camb.info/
} 

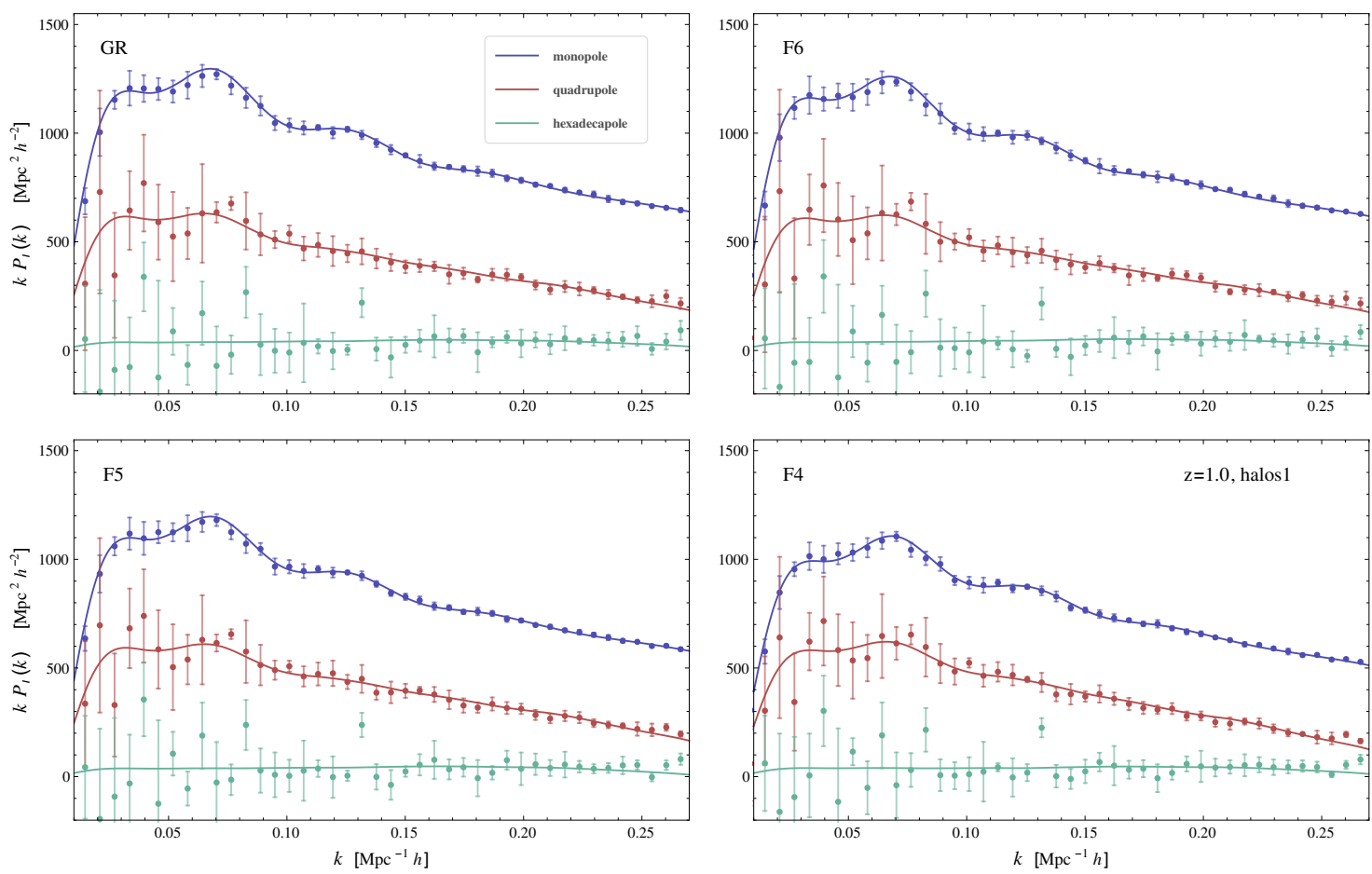

Figure 9. Redshift-space power spectrum monopole, quadrupole, and hexadecapole for halo catalogue $1\left(10^{12}<M_{h}<4.5 \times 10^{12} M_{\odot} h^{-1}\right)$ at redshift $z=1$.

dark matter particle case. We observe very similar trends in the halo catalogue 2 results of figure 8 , and we also find this to be the case with the corresponding comparisons at redshift $z=1$, that are displayed in figures 9 and 10. As a result, these comparisons demonstrate that our model is overall very successful at recovering the anisotropic redshift-space halo PS across a broad range of halo masses, cosmological redshifts and degrees of deviation from the base GR case, for the $f(R)$ HS MG scenario.

In figure 11 we show contour plots for the case of model F5 at $z=0.5$ for the halo catalogue 1 (the rest of the models present similar behavior), showing some degeneracies, particularly for the $b_{1}-c_{0}$ and $b_{1}-c_{2}$ spaces. These are indeed expected since at small scales the EFT parameters, being positive, tend to lower the multipoles, while bigger $b_{1}$ values augment the overall power of the full spectrum. There is also a clear positive correlation between the $c_{0}$ and $c_{2}$ parameters although these affect only the monopole and the quadrupole, respectively; which can be understood as inherited from their degeneracies with the local linear bias $b_{1}$.

Finally, we remark that our approach is close to the work of [73], where the authors find a good accuracy of their perturbative treatment up to $k=0.3 \mathrm{Mpc}^{-1} h$ for redshift $z=0.8$, that is similar to our findings.

\section{Connection to other works}

In this section we would like to make connection to smoothing kernel templates widely used in the literature. 

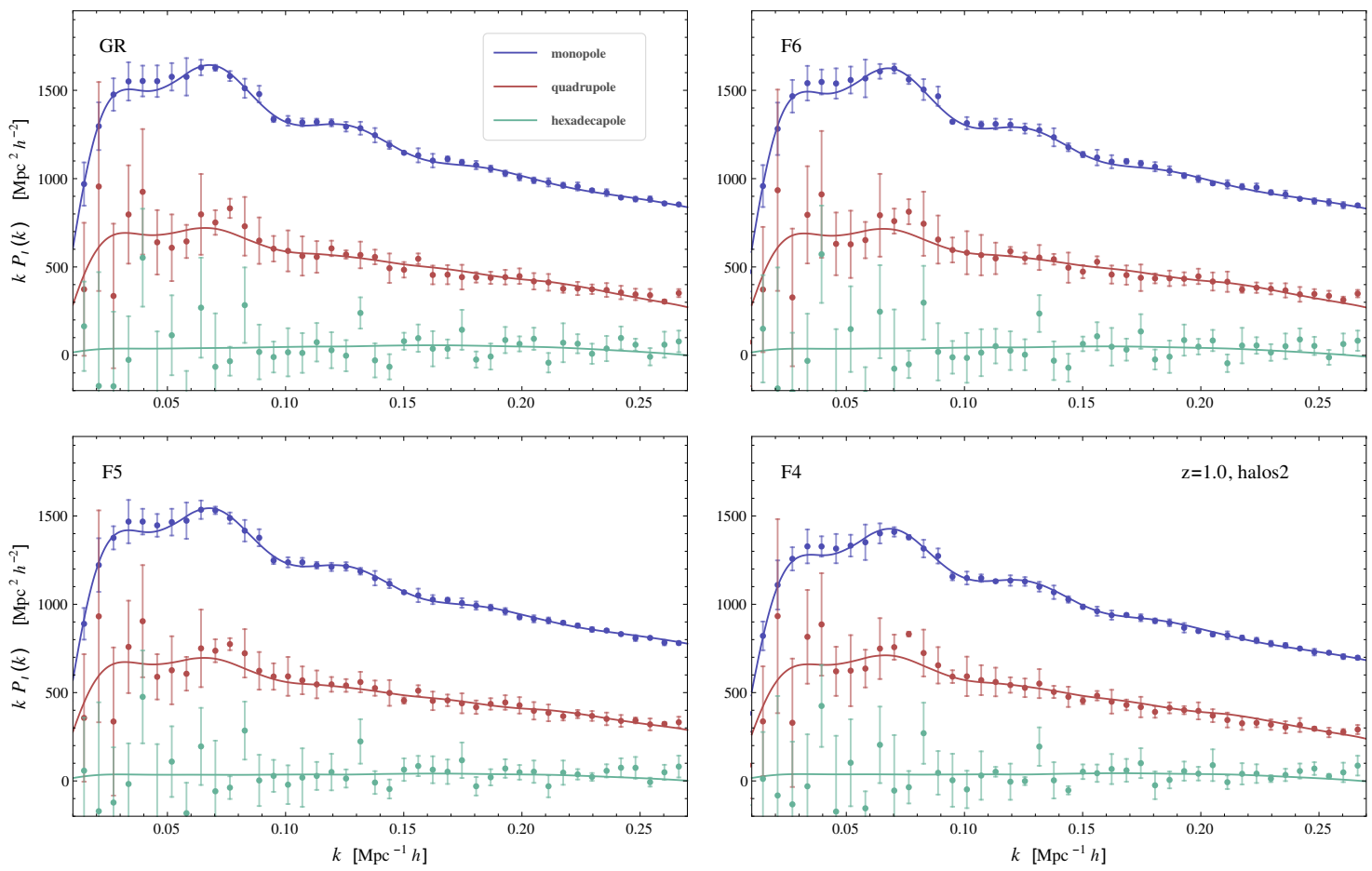

Figure 10. Redshift-space power spectrum multipoles for halo catalogue $2\left(4.5 \times 10^{12}<M_{h}<1 \times 10^{13}\right.$ $\left.M_{\odot} h^{-1}\right)$ at redshift $z=1$.

By taking derivatives to the relation $\left\langle e^{i \mathbf{J} \cdot \mathbf{A}}\right\rangle=\exp \left[\left\langle e^{i \mathbf{J} \cdot \mathbf{A}}\right\rangle_{c}\right]$ between cumulants and moments one arrives to [19, 72]:

$$
\begin{aligned}
& 1+\mathcal{M}(\mathbf{k}, \mathbf{x})=\exp \left[\sum_{n=2}^{\infty} \frac{i^{n}}{n !} k_{i_{1}} \cdots k_{i_{n}}\left\langle\Delta u_{i_{1}} \cdots \Delta u_{i_{n}}\right\rangle_{c}\right]\left\{1+\left\langle\left(\delta_{1}+\delta_{2}\right) e^{i \mathbf{k} \cdot \Delta \mathbf{u}}\right\rangle_{c}\right. \\
& \left.+\left\langle\delta_{1} e^{i \mathbf{k} \cdot \Delta \mathbf{u}}\right\rangle_{c}\left\langle\delta_{2} e^{i \mathbf{k} \cdot \Delta \mathbf{u}}\right\rangle_{c}+\left\langle\delta_{1} \delta_{2} e^{i \mathbf{k} \cdot \Delta \mathbf{u}}\right\rangle_{c}\right\} .
\end{aligned}
$$

The exponential prefactor can be recast as

$$
e^{-\left(k \mu f_{0} \sigma_{v}\right)^{2}} \exp \left[-\frac{1}{2}\left(k_{i} k_{j}\left\langle\Delta u_{i} \Delta u_{j}\right\rangle_{c}-2\left(k \mu f_{0} \sigma_{v}\right)^{2}\right)-\frac{i}{6} k_{i} k_{j} k_{k}\left\langle\Delta u_{i} \Delta u_{j} \Delta u_{k}\right\rangle_{c}+\cdots\right],
$$

such that in the second exponential we removed the zero lag correlator $\left\langle u_{i}(0) u_{j}(0)\right\rangle=$ $2 \sigma_{v}^{2} f_{0}^{2} \hat{n}_{i} \hat{n}_{j}$. We further expand the cumulants in the braces of the RHS of eq. (6.1),

$$
\begin{aligned}
& \left\langle\left(\delta_{1}+\delta_{2}\right) e^{i \mathbf{k} \cdot \Delta \mathbf{u}}\right\rangle_{c}=i k_{i}\left\langle\left(\delta_{1}+\delta_{2}\right) \Delta u_{i}\right\rangle-\frac{1}{2} k_{i} k_{j}\left\langle\left(\delta_{1}+\delta_{2}\right) \Delta u_{i} \Delta u_{j}\right\rangle, \\
& \left\langle\delta_{1} e^{i \mathbf{k} \cdot \Delta \mathbf{u}}\right\rangle_{c}\left\langle\delta_{2} e^{i \mathbf{k} \cdot \Delta \mathbf{u}}\right\rangle_{c}=-k_{i} k_{j}\left\langle\delta_{1} \Delta u_{i}\right\rangle\left\langle\delta_{2} \Delta u_{i}\right\rangle, \\
& \left\langle\delta_{1} \delta_{2} e^{i \mathbf{k} \cdot \Delta \mathbf{u}}\right\rangle_{c}=\left\langle\delta_{1} \delta_{2}\right\rangle+i k_{i}\left\langle\delta_{1} \delta_{2} \Delta u_{i}\right\rangle,
\end{aligned}
$$

valid at 1-loop for primordial Gaussian fields. The idea behind the smoothing kernel is to expand the second exponential in the RHS of eq. (6.2) and the terms $e^{i \mathbf{k} \cdot \Delta \mathbf{u}}$ inside the 


\begin{tabular}{|c|c|c|c|c|c|c|}
\hline \multicolumn{7}{|c|}{ Best Fit Bias and EFT Parameters } \\
\hline Model & $b_{1}$ & $b_{2}$ & $c_{0}$ & $c_{2}$ & $c_{4}$ & $\tilde{c}$ \\
\hline $\mathrm{GR}, z=0.5$, halos 1 & $1.475 \pm 0.004$ & $0.49 \pm 0.01$ & $-28.1 \pm 0.5$ & $-35.3 \pm 0.5$ & $-3.2 \pm 0.1$ & $-0.48 \pm 0.01$ \\
\hline $\mathrm{GR}, z=0.5$, halos 2 & $1.655 \pm 0.003$ & $-1.00 \pm 0.02$ & $-4.3 \pm 0.1$ & $-45.5 \pm 0.5$ & $-6.3 \pm 0.2$ & $-0.50 \pm 0.01$ \\
\hline GR, $z=1.0$, halos 1 & $1.911 \pm 0.003$ & $0.34 \pm 0.01$ & $-16.8 \pm 0.4$ & $-34.3 \pm 0.6$ & $-2.8 \pm 0.1$ & $-0.69 \pm 0.02$ \\
\hline $\mathrm{GR}, z=1.0$, halos 2 & $2.181 \pm 0.002$ & $-0.04 \pm 0.01$ & $-7.6 \pm 0.2$ & $-28.5 \pm 0.7$ & $-1.4 \pm 0.04$ & $-0.92 \pm 0.02$ \\
\hline $\mathrm{F} 6, z=0.5$, halos 1 & $1.415 \pm 0.003$ & $0.91 \pm 0.02$ & $-31.0 \pm 0.5$ & $-26.6 \pm 0.4$ & $-2.2 \pm 0.1$ & $-0.41 \pm 0.01$ \\
\hline $\mathrm{F} 6, z=0.5$, halos 2 & $1.616 \pm 0.003$ & $0.26 \pm 0.01$ & $-27.4 \pm 0.4$ & $-32.2 \pm 0.4$ & $-4.5 \pm 0.1$ & $-0.46 \pm 0.01$ \\
\hline $\mathrm{F} 6, z=1.0$, halos 1 & $1.882 \pm 0.003$ & $0.59 \pm 0.01$ & $-18.8 \pm 0.4$ & $-28.6 \pm 0.5$ & $-0.7 \pm 0.02$ & $-0.70 \pm 0.02$ \\
\hline $\mathrm{F} 6, z=1.0$, halos 2 & $2.173 \pm 0.003$ & $0.77 \pm 0.02$ & $-21.9 \pm 0.5$ & $-23.0 \pm 0.5$ & $-2.3 \pm 0.1$ & $-0.82 \pm 0.02$ \\
\hline $\mathrm{F} 5, z=0.5$, halos 1 & $1.392 \pm 0.003$ & $0.49 \pm 0.01$ & $-28.7 \pm 0.4$ & $-30.2 \pm 0.4$ & $-2.5 \pm 0.1$ & $-0.45 \pm 0.01$ \\
\hline $\mathrm{F} 5, z=0.5$, halos 2 & $1.619 \pm 0.004$ & $0.64 \pm 0.02$ & $-40.0 \pm 0.6$ & $-33.8 \pm 0.5$ & $-3.1 \pm 0.1$ & $-0.48 \pm 0.01$ \\
\hline $\mathrm{F} 5, z=1.0$, halos 1 & $1.818 \pm 0.002$ & $-0.31 \pm 0.01$ & $-9.2 \pm 0.2$ & $-31.3 \pm 0.6$ & $-0.7 \pm 0.02$ & $-0.69 \pm 0.02$ \\
\hline $\mathrm{F} 5, z=1.0$, halos 2 & $2.112 \pm 0.004$ & $1.00 \pm 0.03$ & $-33.4 \pm 0.6$ & $-27.6 \pm 0.6$ & $-4.9 \pm 0.1$ & $-0.57 \pm 0.01$ \\
\hline $\mathrm{F} 4, z=0.5$, halos 1 & $1.270 \pm 0.002$ & $-1.00 \pm 0.02$ & $-8.0 \pm 0.2$ & $-40.1 \pm 0.4$ & $-2.9 \pm 0.1$ & $-0.40 \pm 0.01$ \\
\hline $\mathrm{F} 4, z=0.5$, halos 2 & $1.441 \pm 0.002$ & $-1.00 \pm 0.02$ & $-10.0 \pm 0.2$ & $-44.5 \pm 0.5$ & $-4.2 \pm 0.1$ & $-0.38 \pm 0.01$ \\
\hline $\mathrm{F} 4, z=1.0$, halos 1 & $1.690 \pm 0.004$ & $0.25 \pm 0.01$ & $-23.5 \pm 0.5$ & $-36.9 \pm 0.5$ & $-2.8 \pm 0.1$ & $-0.52 \pm 0.01$ \\
\hline $\mathrm{F} 4, z=1.0$, halos 2 & $1.965 \pm 0.004$ & $0.96 \pm 0.02$ & $-40.2 \pm 0.7$ & $-34.1 \pm 0.7$ & $-5.5 \pm 0.1$ & $-0.52 \pm 0.01$ \\
\hline
\end{tabular}

Table 1. Bias and EFT parameters fitted from simulations and used in figures $7(z=0.5$, halos 1$)$, $8(z=0.5$, halos 2$), 9(z=1$, halos 1$)$, and $8(z=1$, halos 2$)$. The units of parameters $c_{0}, c_{2}$, and $c_{4}$ are $\mathrm{Mpc}^{2} h^{-2}$.

cumulants of the terms within the curly brackets of eq. (6.1). Lengthy but straightforward algebra leads to

$$
\begin{aligned}
1 & +\mathcal{M}(\mathbf{k}, \mathbf{x})=e^{-k^{2} \mu^{2} f_{0}^{2} \sigma_{v}^{2}}\left[1+\sum_{n=0}^{4} \frac{i^{n}}{n !} k_{i_{1}} \cdots k_{i_{n}} \Xi_{i_{1} \ldots i_{n}}^{n}(\mathbf{x})\right. \\
& \left.+\left(k \mu f_{0} \sigma_{v}\right)^{2}\left[\left\langle\delta_{1} \delta_{2}\right\rangle+i k_{i}\left\langle\left(\delta_{1}+\delta_{2}\right) \Delta u_{i}\right\rangle-\frac{1}{2} k_{i} k_{j}\left\langle\Delta u_{i} \Delta u_{j}\right\rangle\right]\right],
\end{aligned}
$$

such that the inverse Fourier transform of eq. (4.5) can be performed analytically to give

$$
P_{s}^{\mathrm{SK}}(\mathbf{k})=e^{-k^{2} \mu^{2} f_{0}^{2} \sigma_{v}^{2}}\left[P_{s}^{K, \mathrm{NL}}(k, \mu)+A(k, \mu)+B(k, \mu)+C(k, \mu)\right] .
$$

Note that by expanding the exponential we recover the moment expansion approach PS [eq. (4.47)]. This model has been used in several works, showing to fit reasonably well to simulations, see e.g. [94], when $\sigma_{v}^{2}$ is considered as a free parameter. This approach has the disadvantage of breaking Galilean invariance that occurs when splitting $\left\langle\Delta u_{i} \Delta u_{j}\right\rangle_{c}=$ $2\left\langle u_{i}(0) u_{j}(0)\right\rangle_{c}-2\left\langle u_{i}(\mathbf{x}) u_{j}(0)\right\rangle_{c}$, and expanding the second term out of the exponential. The consequence of this is the IR divergence we have seen in $C(k, \mu)$, that in the ME expression [eq. (4.47)] is cured by the term $-\left(k \mu f_{0} \sigma_{v}\right)^{2} P_{s}^{K}(k, \mu)$, which is missing in $P_{s}^{\mathrm{SK}}(\mathbf{k})$.

Other smoothing kernel models exist in the literature, but all of them suffer similar problems. The most popular is perhaps the TNS model [22], which seems cannot be deduced 

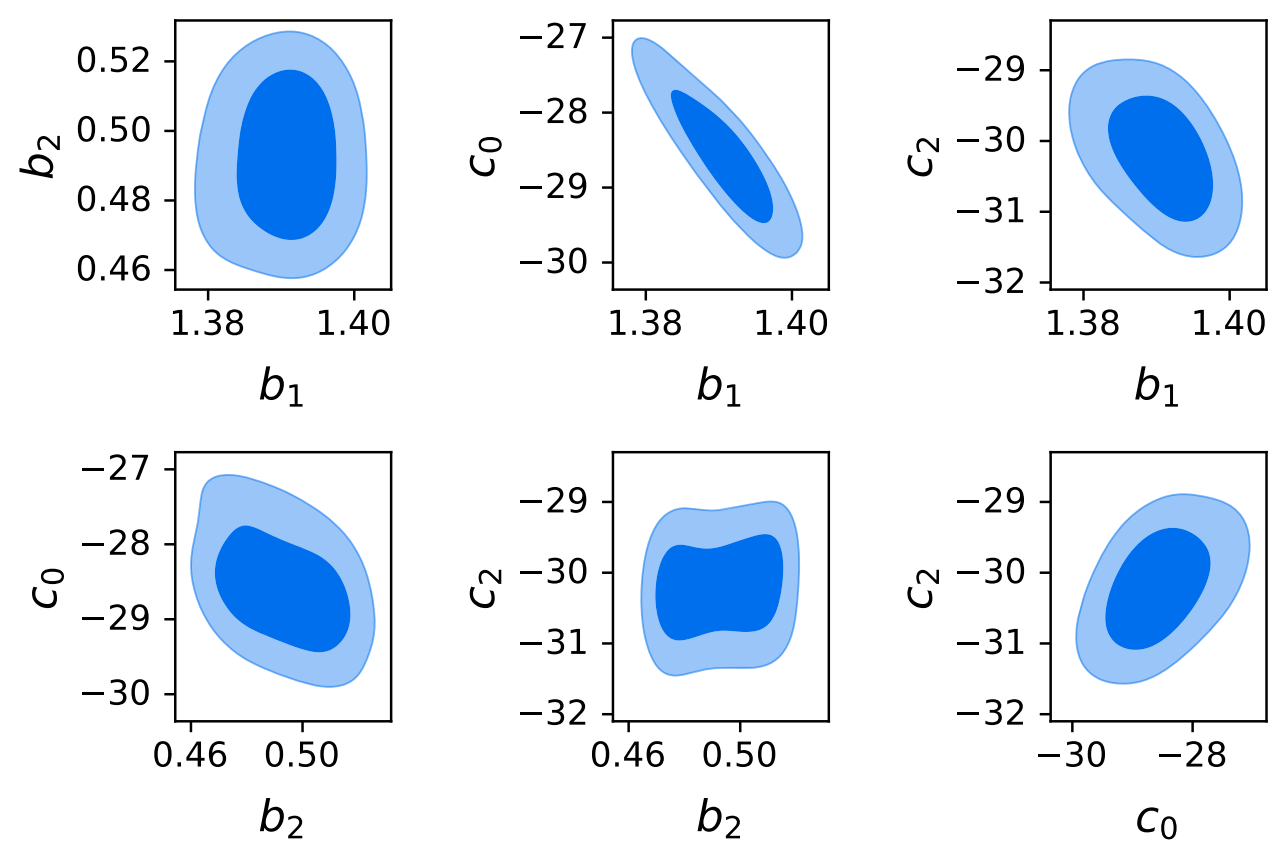

Figure 11. Contour regions for bias and EFT parameters in the cosmology F5, halos 1 at $z=0.5$, corresponding to results in table 1.

within the scheme we adopt, but we can obtain it by simply omitting the function $C(k, \mu)$,

$$
P_{s}^{\mathrm{TNS}}(k, \mu)=e^{-k^{2} \mu^{2} f_{0}^{2} \sigma_{v}^{2}}\left[P_{s}^{K, \mathrm{NL}}(k, \mu)+A(k, \mu)+B(k, \mu)\right] .
$$

This approach does not have the IR divergence, but as we discussed, the $B(k, \mu)$ has terms UV sensitive which should be canceled by the $C(k, \mu)$ function.

Another popular phenomenological model was given by Scoccimarro [19], consisting in neglecting the corrections $A(k, \mu)$ and $D(k, \mu)$,

$$
P_{s}^{\mathrm{Sc} 04}(k, \mu)=e^{-k^{2} \mu^{2} f_{0}^{2} \sigma_{v}^{2}} P_{s}^{K, \mathrm{NL}}(k, \mu) .
$$

hence this model is free UV and IR divergencies, but is incomplete in the sense that not all 1-loop corrections are considered.

\section{$7 \quad$ Summary and Conclusions}

The interplay of high accuracy PT and simulations is an important tool to test both approaches at linear and quasilinear scales. The physical insights from PT and EFT techniques provide the ability to comprehend features in N-point statistics that are valuable to identify effects from different origins, particularly with regards to alternative cosmologies. In the present work we have developed an RSD theory for generalized kernels, beyond EdS, which can be applied to $\Lambda \mathrm{CDM}$ with or without massive neutrinos, MG models, and dark energy sourced by a nontrivial scale dependent Poisson equation. To show its applicability, we focused on the $\Lambda \mathrm{CDM}$ and $\mathrm{HS} f(R)$ models.

The approach developed here is valid for models that comply with eqs. (2.1), (2.5), and (2.6). The source of the Poisson eq. (2.1) defines the alternative DE/gravity model to 
consider. The SPT kernels are then computed by substituting eqs. (2.17) and (2.18) into eqs. (2.10) and (2.11) to solve them iteratively. In this way we found the kernels $F_{n}$ and $G_{n}$ necessary for the computations to 1-loop that are given in the main text for general $A(k, t)$ and $S$ functions, or in appendix B for the specific case of HS. However, we remark that the approach we follow thereafter is independent of the specific kernels. With these at hand, one can readily compute the PS for dark matter perturbations using the expressions at the end of section 2. We then followed ref. [77] to construct the renormalized bias expansion that incorporates new pieces that reflects the complexity of generalized cosmologies, mainly encoded in the kernels themselves. With the biasing theory included, PS for tracers are given in section 3.2 .

In section 4 we developed the main results of this work, that is, a formalism to compute the redshift-space PS for generalized cosmologies. We employed the density-weighted velocity field moments expansion approach [19], based in a formalism that was recently employed for standard cosmology $[72,73]$. We expand the moments in terms of the $I_{n}^{\mathrm{m}}(k)$ functions, eq. (4.9), that had proven to be useful in [22, 94], and here, for our RSD modeling. We computed the different momenta in section 4.2. We then expanded the momenta to arrive to the ME approach PS, eq. (4.47), that is an extension of the known expression to generalized cosmologies. The different pieces of the latter expression are all necessary to have a correct large- and small-scale behavior, as proven in section 4.4. To complete the PS expansion we also computed the EFT counterterms that are necessary to account for backreactions from small-scale physics and also for the damping of the PS along the line-of-sight direction in the particular case of RSD. We also adopted an IR-resummation prescription to correctly model the smearing of $\mathrm{BAO}$ due to non-perturbative large scale bulk flows, that also avoids unphysical large oscillations for small-scale modes.

We finally compared our formalism against simulations, finding very good agreement between our EFT, IR-resummed theory and the state-of-the-art ELEPHANT, MG $N$-body suite of simulations. We made comparisons using two ranges of halo masses at redshifts $z=0.5$ and $z=1$. Within error-bars, we find that the matching is good up to scales $k \sim 0.27 \mathrm{Mpc}^{-1} h$ for the monopole, and slightly smaller $k$ for the quadrupole and hexadecapole. These comparisons demonstrate that our RSD modeling is overall successful at recovering the anisotropic redshiftspace halo PS across a broad range of halo masses, cosmological redshifts and deviations from the base $\Lambda \mathrm{CDM}$ case. They also suggest, we argue, that our model is a promising tool to construct theoretical templates, such as [36, 127, 128], to estimate cosmological parameters using real data obtained from surveys. However, to do this, numerical obstacles may arise because the involved 2-dimensional integrations are constructed from general kernels that are solutions to systems of lengthy differential equations. Such solutions should be obtained at each bin of the 2-dimensional quadratures since these do not have analytical expressions for arbitrary internal momenta, making thus the computations considerably slower than in the corresponding $\Lambda \mathrm{CDM}$ counterpart, where it is customary to use the analytical EdS kernels. Furthermore, the methods to accelerate these integrals by means of FFTlog methods [76, 129, 130] do not apply here, and one has to rely on brute force, which becomes computationally challenging if one needs to span a broad range of parameters in a realistic Monte Carlo Markov Chain analysis. It is important to consider such analysis in future works, since ultimately it will tell us if gravity can be tested using theoretical PT templates and large scales cosmological data from upcoming surveys such as DESI and LSST. That is, without a detailed parameter estimation it is not trivial to conclude to what extent the MG linear PS and kernels are degenerated with biasing, EFT counterterms, and stochasticity. A first step towards this 
direction was recently taken in [131], through a Fisher analysis. On the other hand, from several works (e.g $[28,29]$ ), it is known that the MG screenings are necessary to fit the real space matter power spectrum modeling with simulations. These screenings are non-linear and hence the use of MG kernels $F_{n}$, instead of EdS, is necessary. However, for tracers in redshiftspace the situation is different and it is possible that the MG effects become degenerated with the free parameters at the quasi-linear scales reached by PT/EFT.

To our knowledge, this work presents the most accurate to-date theoretical PT work in the redshift-space PS for MG, being the only one that accounts for beyond linear local biasing, incorporating also EFT counterterms and IR-resummation. Though our theory is based on existing standard cosmology works, mainly [19, 72-75, 77], we organize the theory differently such that, in our opinion, it is easier to compare between different RSD approaches; and further, we find expressions for all the modeling contributions that are valid for kernels beyond EdS.

\section{Acknowledgments}

We thank Oliver Philcox, Shi-Fan Chen, Kazuya Koyama, Gustavo Niz, Matteo Cataneo, Benjamin Bose, and Shun Saito for useful discussions and suggestions. AA, JLCC, and MARM acknowledge support by CONACyT project 283151. AA also acknowledges partial support to CONACyT Ciencia de Frontera grant No. 102958. GV acknowledges financial support by NSF grant AST-1813694. BL is supported by the European Research Council (ERC) through Starting Grant ERC-StG716532-PUNCA, and by the STFC through grants No. ST/T000244/1 and ST/P000541/1. The work of RB is supported by DoE grant DESC0011838, NASA ATP grant 80NSSC18K0695, NASA ROSES grant 12-EUCLID12-0004 and funding related to the Roman High Latitude Survey Science Investigation Team. The simulations described in this work used the DiRAC Data Centric system at Durham University, operated by the Institute for Computational Cosmology on behalf of the STFC DiRAC HPC Facility (www.dirac.ac.uk). This equipment was funded by BIS National E-infrastructure capital grant ST/K00042X/1, STFC capital grants ST/H008519/1, ST/K00087X/1, STFC DiRAC Operations grant ST/K003267/1 and Durham University. DiRAC is part of the National E-Infrastructure.

\section{A $\quad I_{n}^{m}$ functions}

For a rotational scalar function $S(\mathbf{k}, \mathbf{p})=S(k, p, x)$, with $x \equiv \hat{\mathbf{k}} \cdot \hat{\mathbf{p}}$, we use the relation (see also Appendix D of [96])

$$
\int \frac{d^{3} p}{(2 \pi)^{3}}(\hat{\mathbf{p}} \cdot \hat{\mathbf{n}})^{n} S(\mathbf{k}, \mathbf{p})=\sum_{m=0}^{n}(\hat{\mathbf{k}} \cdot \hat{\mathbf{n}})^{m} \int \frac{d^{3} p}{(2 \pi)^{3}} G_{n m}(x) S(k, p, x),
$$

where

$$
\begin{aligned}
G_{n m}(x) & =\sum_{\ell=0}^{n} \frac{\left(1+(-1)^{\ell+n}\right)(2 \ell+1)}{2(1+\ell+n)}\left(\begin{array}{c}
\ell \\
m
\end{array}\right)\left(\begin{array}{c}
2 \ell \\
\ell
\end{array}\right)\left(\begin{array}{c}
\frac{\ell+m-1}{2} \\
\ell
\end{array}\right) \\
& \times{ }_{3} F_{2}\left(\frac{1-\ell}{2},-\frac{\ell}{2}, \frac{1}{2}(-1-\ell-n) ; \frac{1}{2}-\ell, \frac{1}{2}(1-\ell-n) ; 1\right) \mathcal{P}_{\ell}(x)
\end{aligned}
$$

where ${ }_{3} F_{2}(\mathbf{a} ; \mathbf{b} ; z)$ is the generalized hypergeometric function of the kind $(p=3, q=2)$ evaluated at $z=1$ [132], and $\mathcal{P}_{\ell}(x)$ is the Legendre polynomial of degree $\ell$. 
We demonstrate the above expression: starting from (A.1) one arrives to

$$
\sum_{m=0}^{n} \mu^{m} G_{n m}(x)=\sum_{\ell=0}^{n} A_{\ell}^{n} \mathcal{P}_{\ell}(\mu) \mathcal{P}_{\ell}(x)
$$

where $\mathcal{P}_{\ell}$ are the Legendre polynomials and $A_{\ell}^{n}$ is obtained from

$$
y^{n}=\sum_{\ell=0}^{n} A_{\ell}^{n} \mathcal{P}_{\ell}(y),
$$

hence

$$
A_{\ell}^{n}=\frac{\left(1+(-1)^{\ell+n}\right)(2 \ell+1)}{2^{\ell+1}(1+\ell+n)}\left(\begin{array}{c}
2 \ell \\
\ell
\end{array}\right){ }_{3} F_{2}\left(\frac{1-\ell}{2},-\frac{\ell}{2}, \frac{1}{2}(-1-\ell-n) ; \frac{1}{2}-\ell, \frac{1}{2}(1-\ell-n) ; 1\right) .
$$

We further use $\mathcal{P}_{\ell}(\mu)=\sum_{m=0}^{\ell} B_{m}^{\ell} \mu^{m}$, with

$$
B_{m}^{\ell}=2^{\ell}\left(\begin{array}{c}
\ell \\
m
\end{array}\right)\left(\begin{array}{c}
\frac{\ell+m-1}{2} \\
\ell
\end{array}\right) \text {. }
$$

Now, since $A_{\ell}^{n}=0$ for $\ell>n$ and $B_{m}^{\ell}=0$ for $m>\ell$, we arrive to

$$
G_{n m}(x)=\sum_{\ell=0}^{n} A_{\ell}^{n} B_{m}^{\ell} \mathcal{P}_{\ell}(x),
$$

which is eq. (A.2). An equivalent formula for $G_{n m}$ was recently found in [96].

We can derive $G_{n n}=\mathcal{P}_{n}(x)$, and $G_{n m}=0$ for $n<m$ or if $n+m$ is an odd integer. With this formula one obtains all the $G_{n m}(x)$ functions listed in TNS paper [22], with the exception of $G_{55}(x)=\mathcal{P}_{5}(x)$ that in [22] contains a typo. Note also that the indices are inverted $G_{n m}(x)^{\text {Here }}=G_{m n}(x)^{\text {TNS paper }}$.

From eq. (A.1) we can write

$$
\begin{aligned}
\int \frac{d^{3} p}{(2 \pi)^{3}}(\mathbf{p} \cdot \hat{\mathbf{n}})^{n} S(\mathbf{k}, \mathbf{p}) & =\sum_{m=0}^{n} \mu^{m} \int \frac{d^{3} p}{(2 \pi)^{3}} p^{n} G_{n m}(\hat{\mathbf{k}} \cdot \hat{\mathbf{p}}) S(\mathbf{k}, \mathbf{p}) \\
& =\frac{k^{3}}{4 \pi^{2}} \sum_{m=0}^{n} \mu^{m} \int_{0}^{\infty} d r r^{2} \int_{-1}^{1} d x(k r)^{n} G_{n m}(x) S(k, r, x),
\end{aligned}
$$

with $x=\hat{\mathbf{k}} \cdot \hat{\mathbf{p}}$ and $r=p / k$.

\section{A.1 A function}

Function $A(k, \mu)$, defined in eq. (4.39), can be written as

$$
A(k, \mu)=\mu^{2}\left[f_{0} I_{1}^{1, u d d}(k)+f_{0}^{2} I_{1}^{2, \text { uud }}(k)\right]+\mu^{4}\left[f_{0}^{2} I_{2}^{2, \text { uud }}(k)+f_{0}^{3} I_{2}^{3, u u u}(k)\right]+\mu^{6} f_{0}^{3} I_{3}^{3, \text { uuu }}(k),
$$

with the use of eq. (A.1), where

$$
\begin{aligned}
I_{1}^{1, u d d}(k)= & \frac{k^{3}}{4 \pi^{2}} \int_{0}^{\infty} d r \int_{-1}^{1} d x\left\{A_{11}(\mathbf{k}, \mathbf{p}) P_{L}(k)+\tilde{A}_{11}(\mathbf{k}, \mathbf{p}) P_{L}(k r)\right\} \frac{P_{L}(|\mathbf{k}-\mathbf{p}|)}{\left(1+r^{2}-2 r x\right)^{2}} \\
& +\frac{k^{3}}{4 \pi^{2}} \int_{0}^{\infty} d r \int_{-1}^{1} d x a_{11}(\mathbf{k}, \mathbf{p}) P_{L}(k) P_{L}(k r),
\end{aligned}
$$




$$
\begin{aligned}
I_{n}^{2, \text { und }}(k)= & \frac{k^{3}}{4 \pi^{2}} \int_{0}^{\infty} d r \int_{-1}^{1} d x\left\{A_{n 2}(\mathbf{k}, \mathbf{p}) P_{L}(k)+\tilde{A}_{n 2}(\mathbf{k}, \mathbf{p}) P_{L}(k r)\right\} \frac{P_{L}(|\mathbf{k}-\mathbf{p}|)}{\left(1+r^{2}-2 r x\right)^{2}} \\
& +b_{1} \frac{k^{3}}{4 \pi^{2}} \int_{0}^{\infty} d r \int_{-1}^{1} d x a_{n 2}(\mathbf{k}, \mathbf{p}) P_{L}(k) P_{L}(k r), \quad(n=1,2) \\
I_{n}^{3, \text { uиu }}(k)= & \frac{k^{3}}{4 \pi^{2}} \int_{0}^{\infty} d r \int_{-1}^{1} d x\left\{A_{n 3}(\mathbf{k}, \mathbf{p}) P_{L}(k)+\tilde{A}_{n 3}(\mathbf{k}, \mathbf{p}) P_{L}(k r)\right\} \frac{P_{L}(|\mathbf{k}-\mathbf{p}|)}{\left(1+r^{2}-2 r x\right)^{2}} \\
& +b_{1} \frac{k^{3}}{4 \pi^{2}} \int_{0}^{\infty} d r \int_{-1}^{1} d x a_{n 3}(\mathbf{k}, \mathbf{p}) P_{L}(k) P_{L}(k r), \quad(n=2,3)
\end{aligned}
$$

with $A_{a b}$ and $a_{a b}$ functions

$$
\begin{aligned}
\frac{A_{11}(\mathbf{k}, \mathbf{p})}{\left(1+r^{2}-2 r x\right)^{2}}= & 2 b_{1}^{2} r x G_{2}(-\mathbf{k}, \mathbf{k}-\mathbf{p}) \\
+ & b_{1}^{2} \frac{2 r^{2}(1-r x)}{1+r^{2}-2 r x} G_{1}(|\mathbf{k}-\mathbf{p}|)\left\{F_{2}(-\mathbf{k}, \mathbf{k}-\mathbf{p})+\frac{b_{2}}{2 b_{1}}+\frac{b_{s}}{b_{1}} S(-\mathbf{k}, \mathbf{k}-\mathbf{p})\right\}, \\
\frac{\tilde{A}_{11}(\mathbf{k}, \mathbf{p})}{\left(1+r^{2}-2 r x\right)^{2}}= & 2 b_{1}^{2}\left[G_{1}(p) r x+G_{1}(|\mathbf{k}-\mathbf{p}|) \frac{r^{2}(1-r x)}{1+r^{2}-2 r x}\right] \\
& \times\left\{F_{2}(\mathbf{p}, \mathbf{k}-\mathbf{p})+\frac{b_{2}}{2 b_{1}}+\frac{b_{s}}{b_{1}} S(\mathbf{p}, \mathbf{k}-\mathbf{p})\right\} \\
a_{11}(\mathbf{k}, \mathbf{p})= & 2 b_{1}^{2} G_{2}(-\mathbf{k},-\mathbf{p}) \frac{r^{2}(1-r x)}{1+r^{2}-2 r x} \\
& +2 b_{1}^{2} r x G_{1}(p)\left\{F_{2}(-\mathbf{k}, \mathbf{p})+\frac{b_{2}}{2 b_{1}}+\frac{b_{s}}{b_{1}} S(-\mathbf{k}, \mathbf{p})\right\}
\end{aligned}
$$

$$
\begin{aligned}
\frac{A_{12}(\mathbf{k}, \mathbf{p})}{\left(1+r^{2}-2 r x\right)^{2}} & =-b_{1} \frac{r^{2}\left(1-x^{2}\right)}{1+r^{2}-2 r x} G_{2}(-\mathbf{k}, \mathbf{k}-\mathbf{p}) G_{1}(|\mathbf{k}-\mathbf{p}|), \\
\frac{\tilde{A}_{12}(\mathbf{k}, \mathbf{p})}{\left(1+r^{2}-2 r x\right)^{2}} & =-b_{1} \frac{r^{2}\left(1-x^{2}\right)}{1+r^{2}-2 r x} G_{1}(p) G_{1}(|\mathbf{k}-\mathbf{p}|)\left\{F_{2}(\mathbf{p}, \mathbf{k}-\mathbf{p})+\frac{b_{2}}{2 b_{1}}+\frac{b_{s}}{b_{1}} S(\mathbf{p}, \mathbf{k}-\mathbf{p})\right\} \\
a_{12}(\mathbf{k}, \mathbf{p}) & =-b_{1} \frac{r^{2}\left(1-x^{2}\right)}{1+r^{2}-2 r x} G_{2}(\mathbf{p},-\mathbf{k}) G_{1}(p),
\end{aligned}
$$




$$
\begin{aligned}
& \frac{A_{22}(k, x, r)}{\left(1+r^{2}-2 r x\right)^{2}}=b_{1}\left[\frac{r^{2}\left(1-3 x^{2}\right)+2 x r}{1+r^{2}-2 r x} G_{1}(|\mathbf{k}-\mathbf{p}|)+2 x r G_{1}(k)\right] G_{2}(-\mathbf{k}, \mathbf{k}-\mathbf{p}) \\
& +b_{1} \frac{2 r^{2}(1-r x)}{1+r^{2}-2 r x} G_{1}(|\mathbf{k}-\mathbf{p}|) G_{1}(k)\left\{F_{2}(-\mathbf{k}, \mathbf{k}-\mathbf{p})+\frac{b_{2}}{2 b_{1}}+\frac{b_{s}}{b_{1}} S(\mathbf{k}-\mathbf{p},-\mathbf{k})\right\}, \\
& \frac{\tilde{A}_{22}(k, x, r)}{\left(1+r^{2}-2 r x\right)^{2}}=b_{1}\left[\frac{2 r^{2}(1-r x)}{1+r^{2}-2 r x} G_{1}(|\mathbf{k}-\mathbf{p}|)+2 x r G_{1}(p)\right] G_{2}(\mathbf{p}, \mathbf{k}-\mathbf{p}) \\
& +b_{1} \frac{r^{2}\left(1-3 x^{2}\right)+2 x r}{1+r^{2}-2 r x} G_{1}(|\mathbf{k}-\mathbf{p}|) G_{1}(p)\left\{F_{2}(\mathbf{p}, \mathbf{k}-\mathbf{p})+\frac{b_{2}}{2 b_{1}}+\frac{b_{s}}{b_{1}} S(\mathbf{p}, \mathbf{k}-\mathbf{p})\right\}, \\
& a_{22}(k, x, r)=b_{1}\left[\frac{r^{2}\left(1-3 x^{2}\right)+2 x r}{1+r^{2}-2 r x} G_{1}(p)+\frac{2 r^{2}(1-r x)}{1+r^{2}-2 r x} G_{1}(k)\right] G_{2}(-\mathbf{k}, \mathbf{p}) \\
& +2 b_{1} x r G_{1}(p) G_{1}(k)\left\{F_{2}(-\mathbf{k}, \mathbf{p})+\frac{b_{2}}{2 b_{1}}+\frac{b_{s}}{b_{1}} S(\mathbf{p},-\mathbf{k})\right\} . \\
& \frac{A_{23}(k, x, r)}{\left(1+r^{2}-2 r x\right)^{2}}=\frac{r^{2}\left(x^{2}-1\right)}{1+r^{2}-2 r x} G_{2}(-\mathbf{k}, \mathbf{k}-\mathbf{p}) G_{1}(k) G_{1}(|\mathbf{k}-\mathbf{p}|), \\
& \frac{\tilde{A}_{23}(k, x, r)}{\left(1+r^{2}-2 r x\right)^{2}}=\frac{r^{2}\left(x^{2}-1\right)}{1+r^{2}-2 r x} G_{2}(\mathbf{p}, \mathbf{k}-\mathbf{p}) G_{1}(p) G_{1}(|\mathbf{k}-\mathbf{p}|), \\
& a_{23}(k, x, r)=\frac{r^{2}\left(x^{2}-1\right)}{1+r^{2}-2 r x} G_{2}(-\mathbf{k}, \mathbf{p}) G_{1}(k) G_{1}(p) . \\
& \frac{A_{33}(k, x, r)}{\left(1+r^{2}-2 r x\right)^{2}}=\frac{r^{2}\left(1-3 x^{2}\right)+2 r x}{1+r^{2}-2 r x} G_{2}(-\mathbf{k}, \mathbf{k}-\mathbf{p}) G_{1}(k) G_{1}(|\mathbf{k}-\mathbf{p}|), \\
& \frac{\tilde{A}_{33}(k, x, r)}{\left(1+r^{2}-2 r x\right)^{2}}=\frac{r^{2}\left(1-3 x^{2}\right)+2 r x}{1+r^{2}-2 r x} G_{2}(\mathbf{p}, \mathbf{k}-\mathbf{p}) G_{1}(p) G_{1}(|\mathbf{k}-\mathbf{p}|), \\
& a_{33}(k, x, r)=\frac{r^{2}\left(1-3 x^{2}\right)+2 r x}{1+r^{2}-2 r x} G_{2}(-\mathbf{k}, \mathbf{p}) G_{1}(k) G_{1}(p) .
\end{aligned}
$$

All these functions $A_{a b}$ reduce to the corresponding in TNS paper for the unbiased case $\left(b_{1}=1, b_{2}=b_{s}=0\right)$ and with EdS kernels $G_{1}, G_{2}$, and $F_{2}$.

\section{A.2 D function}

The $D(k, \mu)$ function in eq. (4.42) is

$$
\begin{aligned}
D(k, \mu)= & \mu^{2}\left[f_{0}^{2} I_{1}^{2, \text { uиdd }}(k)+f_{0}^{3} I_{1}^{3, \text { uии }}(k)+f_{0}^{4} I_{1}^{4, \text { uиии }}(k)\right] \\
& +\mu^{4}\left[f_{0}^{2} I_{2}^{2, \text { uudd }}(k)+f_{0}^{3} I_{2}^{3, \text { uиud }}(k)+f_{0}^{4} I_{2}^{4, \text { uиии }}(k)\right] \\
& +\mu^{6}\left[f_{0}^{3} I_{3}^{3, \text { uuиd }}(k)+f_{0}^{4} I_{3}^{4, \text { uиии }}(k)\right]+\mu^{8} f_{0}^{4} I_{4}^{4, \text { uиии }}(k),
\end{aligned}
$$

with

$$
\begin{aligned}
& I_{n}^{2, \text { uиdd }}(k)=I_{n B}^{2, \text { uudd }}(k)+I_{n C}^{2, \text { uиdd }}(k)-\delta_{n 1} k^{2} \sigma_{v}^{2} P_{\delta \delta}(k), \\
& I_{n}^{3, \text { uиud }}(k)=I_{n B}^{3, \text { uuиd }}(k)+I_{n C}^{3, \text { uиud }}(k)-\delta_{n 2} 2 k^{2} \sigma_{v}^{2} P_{\delta \theta}(k), \\
& I_{n}^{4, \text { uиии }}(k)=I_{n B}^{4, \text { uиии }}(k)+I_{n C}^{4, \text { uиuи }}(k)-\delta_{n 3} k^{2} \sigma_{v}^{2} P_{\theta \theta}(k),
\end{aligned}
$$


according to the splitting $D=B+C-\left(k \sigma_{v} f_{0} \mu\right)^{2} P_{s}^{K}$.

$$
I_{n B}^{2, u u d d}(k)=\frac{k^{3}}{4 \pi^{2}} \int_{0}^{\infty} d r \int_{-1}^{1} d x B_{11}^{n}(r, x) \frac{P_{\delta \theta}(k r) P_{\delta \theta}(|\mathbf{k}-\mathbf{p}|)}{1+r^{2}-2 r x},
$$

with

$$
\begin{gathered}
B_{11}^{1}(r, x)=\frac{r^{2}}{2}\left(x^{2}-1\right), \quad B_{11}^{2}(r, x)=\frac{r}{2}\left(r-3 r x^{2}+2 x\right) . \\
I_{1 C}^{2, u u d d}(k)=\frac{k^{3}}{4 \pi^{2}} \int_{0}^{\infty} d r \int_{-1}^{1} d x \frac{1}{4}\left(1-x^{2}\right)\left\{P_{\delta \delta}\left(k \sqrt{1+r^{2}-2 r x}\right) P_{\theta \theta}(k r)\right. \\
\left.+r^{4} \frac{P_{\delta \delta}(k r) P_{\theta \theta}\left(k \sqrt{1+r^{2}-2 r x}\right)}{\left(1+r^{2}-2 r x\right)^{2}}\right\}, \\
I_{2 C}^{2, u u d d}(k)=\frac{k^{3}}{4 \pi^{2}} \int_{0}^{\infty} d r \int_{-1}^{1} d x\left\{\frac{1}{4}\left(3 x^{2}-1\right) P_{\delta \delta}\left(k \sqrt{1+r^{2}-2 r x}\right) P_{\theta \theta}(k r)\right. \\
\left.+\frac{r^{2}}{4}\left[2-4 r x+r^{2}\left(3 x^{2}-1\right)\right] \frac{P_{\delta \delta}(k r) P_{\theta \theta}\left(k \sqrt{1+r^{2}-2 r x}\right)}{\left(1+r^{2}-2 r x\right)^{2}}\right\} .
\end{gathered}
$$

We note these expressions have no counterpart in $B$, because the product here is $P_{\delta \delta} P_{\theta \theta}$, while the products for functions $B$ are $P_{\delta \theta} P_{\delta \theta}, P_{\delta \theta} P_{\theta \theta}$, and $P_{\theta \theta} P_{\theta \theta}$. Therefore, functions $C_{11}^{1}$ or $C_{11}^{2}$, with the same meaning as those relative to $B$, do not exist.

Functions with $\mathrm{m}=3$ are

$$
\begin{aligned}
I_{n C}^{3, \text { uиud }}(k)= & -\frac{k^{3}}{4 \pi^{2}} \int_{0}^{\infty} d r \int_{-1}^{1} d x C_{21}^{n}(k, r, x) \frac{P_{\theta \theta}\left(k \sqrt{1+r^{2}-2 r x}\right) P_{\delta \theta}(k r)}{\left(1+r^{2}-2 r x\right)^{2}} \\
& -\frac{k^{3}}{4 \pi^{2}} \int_{0}^{\infty} d r \int_{-1}^{1} d x C_{12}^{n}(k, r, x) \frac{P_{\delta \theta}\left(k \sqrt{1+r^{2}-2 r x}\right) P_{\theta \theta}(k r)}{\left(1+r^{2}-2 r x\right)}, \\
I_{n B}^{3, \text { uuud }}(k)= & -\frac{k^{3}}{4 \pi^{2}} \int_{0}^{\infty} d r \int_{-1}^{1} d x B_{21}^{n}(k, r, x) \frac{P_{\theta \theta}\left(k \sqrt{1+r^{2}-2 r x}\right) P_{\delta \theta}(k r)}{\left(1+r^{2}-2 r x\right)^{2}} \\
& -\frac{k^{3}}{4 \pi^{2}} \int_{0}^{\infty} d r \int_{-1}^{1} d x B_{12}^{n}(k, r, x) \frac{P_{\delta \theta}\left(k \sqrt{1+r^{2}-2 r x}\right) P_{\theta \theta}(k r)}{\left(1+r^{2}-2 r x\right)},
\end{aligned}
$$

with

$$
\begin{aligned}
& C_{21}^{1}(r, x)=-\frac{3 r^{4}}{8}\left(x^{2}-1\right)^{2}=-B_{21}^{1}(r, x), \\
& C_{12}^{1}(r, x)=-\frac{3 r^{2}}{8}\left(x^{2}-1\right)^{2}=-B_{21}^{1}(r, x),
\end{aligned}
$$


showing that indeed, $I_{1 D}^{3, \text { uuud }}(k)=I_{1 B}^{3, \text { uuud }}(k)+I_{1 C}^{3, \text { uuud }}(k)=0$, such that there is not $f_{0}^{3} \mu^{2}$ term in $D(k, \mu)$ function. The rest of the functions are

$$
\begin{aligned}
& C_{21}^{2}(r, x)=-\frac{r^{2}}{4}\left(1-x^{2}\right)\left(2-3 r^{2}-12 r x+15 r^{2} x^{2}\right), \\
& C_{12}^{2}(r, x)=-\frac{1}{4}\left(1-x^{2}\right)\left(2-3 r^{2}-12 r x+15 r^{2} x^{2}\right), \\
& B_{21}^{2}(r, x)=\frac{3}{4} r^{2}\left(-1+x^{2}\right)\left(-2+r^{2}+6 r x-5 r^{2} x^{2}\right), \\
& B_{12}^{2}(r, x)=-\frac{3}{4} r\left(-1+x^{2}\right)\left(-r-2 x+5 r x^{2}\right),
\end{aligned}
$$

and

$$
\begin{aligned}
& C_{21}^{3}(r, x)=-\frac{r^{2}}{8}\left(-4+3 r^{2}+24 r x+12 x^{2}-30 r^{2} x^{2}-40 r x^{3}+35 r^{2} x^{4}\right), \\
& C_{12}^{3}(r, x)=-\frac{1}{8}\left(-4+3 r^{2}+24 r x+12 x^{2}-30 r^{2} x^{2}-40 r x^{3}+35 r^{2} x^{4}\right), \\
& B_{21}^{3}(r, x)=\frac{r}{8}\left\{-8 x+r\left[-12+36 x^{2}+12 r x\left(3-5 x^{2}\right)+r^{2}\left(3-30 x^{2}+35 x^{4}\right)\right]\right\}, \\
& B_{12}^{3}(r, x)=\frac{r}{8}\left[4 x\left(3-5 x^{2}\right)+r\left(3-30 x^{2}+35 x^{4}\right)\right] .
\end{aligned}
$$

Functions with $\mathrm{m}=4$ are

$$
\begin{aligned}
& I_{n B}^{4, \text { ичии }}(k)=\frac{k^{3}}{4 \pi^{2}} \int_{0}^{\infty} d r \int_{-1}^{1} d x B_{22}^{n}(r, x) \frac{P_{\theta \theta}(k r) P_{\theta \theta}\left(k \sqrt{1+r^{2}-2 r x}\right)}{\left(1+r^{2}-2 r x\right)^{2}}, \\
& I_{n C}^{4, \text { ичии }}(k)=\frac{k^{3}}{4 \pi^{2}} \int_{0}^{\infty} d r \int_{-1}^{1} d x C_{22}^{n}(r, x) \frac{P_{\theta \theta}(k r) P_{\theta \theta}\left(k \sqrt{1+r^{2}-2 r x}\right)}{\left(1+r^{2}-2 r x\right)^{2}},
\end{aligned}
$$

with

$$
\begin{aligned}
C_{22}^{1}= & -\frac{5 r^{4}}{16}\left(-1+x^{2}\right)^{3}=-B_{22}^{1}, \\
C_{22}^{2}= & \frac{3 r^{2}}{16}\left(-1+x^{2}\right)^{2}\left(7-5 r^{2}-30 r x+35 r^{2} x^{2}\right), \\
C_{22}^{3}=-\frac{1}{16}\left(-1+x^{2}\right)\left[4+3 r\left\{-16 x+r\left[-14+70 x^{2}+20 r x\left(3-7 x^{2}\right)\right.\right.\right. & \left.\left.\left.\quad+5 r^{2}\left(1-14 x^{2}+21 x^{4}\right)\right]\right\}\right], \\
C_{22}^{4}=\frac{1}{16}[ & -4+12 x^{2}+16 r x\left(3-5 x^{2}\right)+7 r^{2}\left(3-30 x^{2}+35 x^{4}\right) \\
& \left.\quad-6 r^{3} x\left(15-70 x^{2}+63 x^{4}\right)+r^{4}\left\{-5+21 x^{2}\left(5-15 x^{2}+11 x^{4}\right)\right\}\right],
\end{aligned}
$$

and

$$
\begin{aligned}
B_{22}^{2}= & -\frac{3 r^{2}}{16}\left(-1+x^{2}\right)^{2}\left(6-5 r^{2}-30 r x+35 r^{2} x^{2}\right), \\
B_{22}^{3}= & \frac{3 r}{16}\left(x^{2}-1\right)\left[-8 x+r\left\{-12+60 x^{2}+20 r x\left(3-7 x^{2}\right)+5 r^{2}\left(1-14 x^{2}+21 x^{4}\right)\right\}\right], \\
B_{22}^{4}= & \frac{r}{16}\left[8 x\left(-3+5 x^{2}\right)-6 r\left(3-30 x^{2}+35 x^{4}\right)+6 r^{2} x\left(15-70 x^{2}+63 x^{4}\right)\right. \\
& \quad+r^{3}\left\{\left(5-21 x^{2}\left(5-15 x^{2}+11 x^{4}\right)\right\}\right] .
\end{aligned}
$$


The relation $C_{22}^{1}+B_{22}^{1}=0$ implies that, $I_{1}^{4}(k)=0$, such that there is not $f_{0}^{4} \mu^{2}$ term in $D(k, \mu)$ function.

We stress out that all our functions $B_{a b}^{n}$ are identical to those presented in TNS paper, this is because all are constructed out of products of correlators of linear fields. The only difference is that a factor $f(k) / f_{0}$ is introduced each time a velocity field $\theta$ is present. The biasing of these functions is implicit in the power spectra $P_{\delta \delta}$ and $P_{\theta \theta}$ and cross-power spectrum $P_{\delta \theta}$. Meaning that

$$
B\left(k, \mu ; f_{0}\right) \longrightarrow b_{1}^{4} B\left(k, \mu, f_{0} / b_{1}\right), \quad C\left(k, \mu ; f_{0}\right) \longrightarrow b_{1}^{4} C\left(k, \mu, f_{0} / b_{1}\right) .
$$

As we mentioned in section 3 , we restrict the inclusion of curvature bias to $\mathcal{O}\left(P_{L}\right)$ terms, so it does not appear in the above expressions.

\section{B On the $f(R)$ Hu-Sawicki model}

Throughout this work we apply our results to the Hu-Sawicki $f(R)$ model [133], with $n=1$ and amplitude $f_{R 0}=-10^{-6},-10^{-5}-10^{-4}$, called F6, F5 and F4, respectively. We stress that results for other gravity models are straightforward to develop, following our formalism. Models defined in the Einstein frame can be put also in our frame by using field redefinitions; see [31]. In this appendix we summarize the main aspects needed from the HS models.

A general $f(R)$ action is written as

$$
S=\int d^{4} x \sqrt{-g}(R+f(R))+\int d^{4} x \sqrt{-g} \mathcal{L}_{m} .
$$

Variations with respect to the metric of this action lead to the field equations

$$
G_{\mu \nu}+f_{R} R_{\mu \nu}-\nabla_{\mu} \nabla_{\nu} f_{R}-\left(\frac{f}{2}-\square f_{R}\right) g_{\mu \nu}=8 \pi G T_{\mu \nu}
$$

where $f_{R} \equiv \frac{d f(R)}{d R}$, that represents the scalar field degree of freedom of the theory. By taking the trace to this equation one obtains

$$
3 \square f_{R}=R\left(1-f_{R}\right)+2 f-8 \pi G \rho,
$$

where we use a dark matter fluid with $T_{\mu}^{\mu}=-\rho$.

Considering a perturbed Friedmann-Robertson-Walker line element

$$
d s^{2}=-(1+2 \Phi) d t^{2}+a(t)^{2}(1-2 \Psi) d \mathbf{x}^{2}
$$

the fluid perturbation $\Delta \rho=\bar{\rho} \delta$ and the associated scalar field perturbation $\delta f_{R}=f_{R}-\bar{f}_{R}$, $R=\bar{R}+\delta R$, where the bar indicates background quantities and $\bar{R} \equiv R\left(\bar{f}_{R}\right)$, the perturbative field equations in Fourier space are [28, 29]:

$$
\begin{aligned}
-\frac{k^{2}}{a^{2}} \Phi & =4 \pi G \bar{\rho} \delta+\frac{1}{2} \frac{k^{2}}{a^{2}} \delta f_{R}, \\
3 \frac{k^{2}}{a^{2}} \delta f_{R} & =8 \pi G \bar{\rho} \delta-M_{1}(k) \delta f_{R}-\delta \mathcal{I}\left(\delta f_{R}\right), \\
\Psi-\Phi & =\delta f_{R},
\end{aligned}
$$


where $m \equiv\left(M_{1} / 3\right)^{1 / 2}$ represents the mass of scalar field. On scales larger than $m^{-1}$, the scalar field does not propagate and one recovers the $\Lambda$ CDM model. On the other hand, on small scales $\left(k \rightarrow \infty, \mu \rightarrow 4 / 3\right.$, see eq. B.8), so gravity is enhanced by one third, $G_{\text {eff }} \rightarrow(4 / 3) G$.

Substituting eq. (B.6) in (B.5), one arrives to eq. (2.1) identifying the functions:

$$
\begin{aligned}
\mu(k, t) & =1+\frac{k^{2} / a^{2}}{3 k^{2} / a^{2}+M_{1}(k)}, \\
S(\mathbf{k}) & =-\frac{1}{2} \frac{k^{2} / a^{2}}{3 k^{2} / a^{2}+M_{1}(k)} \delta \mathcal{I},
\end{aligned}
$$

$\delta \mathcal{I}$ represents nonlinear interactions which can be expanded as:

$$
\begin{aligned}
\delta \mathcal{I}\left(\delta f_{R}\right)= & \frac{1}{2} \int \frac{d^{3} k_{1} d^{3} k_{2}}{(2 \pi)^{3}} \delta_{D}\left(\mathbf{k}-\mathbf{k}_{12}\right) M_{2}\left(\mathbf{k}_{1}, \mathbf{k}_{2}\right) \delta f_{R}\left(\mathbf{k}_{1}\right) \delta f_{R}\left(\mathbf{k}_{2}\right) \\
& +\frac{1}{6} \int \frac{d^{3} k_{1} d^{3} k_{2} d^{3} k_{3}}{(2 \pi)^{6}} \delta_{D}\left(\mathbf{k}-\mathbf{k}_{123}\right) M_{3}\left(\mathbf{k}_{1}, \mathbf{k}_{2}, \mathbf{k}_{3}\right) \delta f_{R}\left(\mathbf{k}_{1}\right) \delta f_{R}\left(\mathbf{k}_{2}\right) \delta f_{R}\left(\mathbf{k}_{3}\right)+\cdots
\end{aligned}
$$

where the functions $M_{i}$ are in general scale and time dependent and are determined by the $f(R)$ model.

Giving eq. (B.8), we can now determine $A(k, t)$ using eq. (2.2), as

$$
A(k, t)=4 \pi G \bar{\rho} \mu(k, t)=4 \pi G \bar{\rho}\left(1+\frac{k^{2} / a^{2}}{3 k^{2} / a^{2}+M_{1}(k)}\right),
$$

that enters in eq. (2.15) to determine the growth function to first order $D_{+}(k, t)$. It only remains to determine the functions $M_{i}$ to have all the needed functions to be able to integrate the system. These functions are given for $f(R)$ gravity by

$$
\delta R=\sum_{i} \frac{1}{n !} M_{n}\left(\delta f_{R}\right)^{n},\left.\quad M_{n} \equiv \frac{d^{n} R\left(f_{R}\right)}{d f_{R}^{n}}\right|_{f_{R}=\bar{f}_{R}} .
$$

For the $n=1$ HS model can be expressed as:

$$
f(R)=-\frac{c_{1} R}{c_{2} R / M^{2}+1} .
$$

In order to have an effective $\Lambda$ CDM model at background level, the energy scale is chosen to be $M^{2}=H_{0}^{2} \Omega_{m 0}$ and $c_{1} / c_{2}=6 \Omega_{\Lambda} / \Omega_{m 0}$.

The functions $M_{1}, M_{2}$ and $M_{3}$ are:

$$
\begin{gathered}
M_{1}(a)=\frac{3}{2} \frac{H_{0}^{2}}{\left|f_{R 0}\right|} \frac{\left(\Omega_{m 0} a^{-3}+4 \Omega_{\Lambda}\right)^{3}}{\left(\Omega_{m 0}+4 \Omega_{\Lambda}\right)^{2}}, \\
M_{2}(a)=\frac{9}{4} \frac{H_{0}^{2}}{\left|f_{R 0}\right|^{2}} \frac{\left(\Omega_{m 0} a^{-3}+4 \Omega_{\Lambda}\right)^{5}}{\left(\Omega_{m 0}+4 \Omega_{\Lambda}\right)^{4}}, \\
M_{3}(a)=\frac{45}{8} \frac{H_{0}^{2}}{\left|f_{R 0}\right|^{3}} \frac{\left(\Omega_{m 0} a^{-3}+4 \Omega_{\Lambda}\right)^{7}}{\left(\Omega_{m 0}+4 \Omega_{\Lambda}\right)^{6}} .
\end{gathered}
$$


These functions depend only on the background evolution since they are the coefficients of the expansion of a scalar field potential about its background value, see eq. (B.12).

The second order source entering eq. (2.23) becomes

$$
\mathcal{S}^{(2)}\left(\mathbf{k}_{1}, \mathbf{k}_{2}\right)=-\left(\frac{2 A_{0}}{3}\right)^{2} \frac{M_{2}\left(\mathbf{k}_{1}, \mathbf{k}_{2}\right) k^{2} / a^{2}}{6 \Pi(k) \Pi\left(k_{1}\right) \Pi\left(k_{2}\right)},
$$

where $\Pi(k) \equiv \frac{1}{3 a^{2}}\left(3 k^{2}+M_{1} a^{2}\right)$.

\section{SPT generalized kernels}

In SPT the expansion is performed directly to the overdensity and velocity fields, $\delta=\delta^{(1)}+$ $\delta^{(2)}+\cdots$ and $\theta=\theta^{(1)}+\theta^{(2)}+\cdots$, which we have written as a Taylor Fourier expansion in eqs. (2.17) and (2.18). The $F_{n}$ and $G_{n}$ kernels are usually obtained by solving iteratively continuity and Euler equations, eqs. (2.10) and (2.11). In this notation, the linear growth functions $D_{+}$are kept attached to the linear fields because they are scale dependent and cannot be pulled out of the integral; and the $G_{n}$ kernels carry the linear growth rate $f(\mathbf{k}, t)$. Our notation coincides with that of ref. [30] except for a minus sign in $G_{n}$, but differs from the most used notations that factorize the $f$ factors.

Instead of solving for $F_{n}$ and $G_{n}$ directly, we obtain them from known LPT kernels, using the mappings developed in [83]. In LPT one follows the trajectories $\mathbf{x}$ of particles initially located at a Lagrangian position $\mathbf{q}$,

$$
x^{i}(\mathbf{q}, t)=q^{i}+\Psi^{i}(\mathbf{q}, t),
$$

with the Lagrangian displacement vector field $\Psi$ which in PT is written as

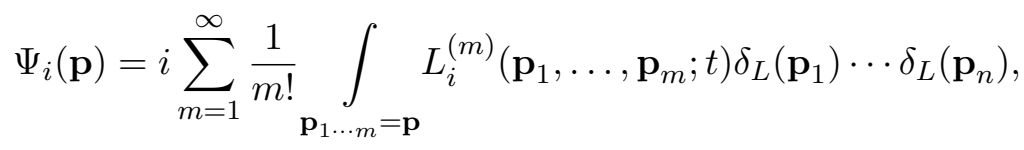

with $L_{i}^{(m)}$ the LPT kernels. For $F_{n}$ we have the relation [83]

$$
F_{n}\left(\mathbf{k}_{1}, \ldots, \mathbf{k}_{n}\right)=\sum_{\ell=1}^{n} \sum_{m_{1}+\cdots+m_{\ell}=n} \frac{k_{i_{1}} \cdots k_{i_{\ell}}}{\ell ! m_{1} ! \cdots m_{\ell} !} L_{i_{i}}^{\left(m_{1}\right)}\left(\mathbf{k}_{1}, \ldots, \mathbf{k}_{m_{1}}\right) \cdots L_{i_{\ell}}^{\left(m_{\ell}\right)}\left(\mathbf{k}_{m_{\ell-1}} \cdots, \mathbf{k}_{m_{\ell}}\right) .
$$

Once symmetrized, for $F_{2}$ and $G_{2}$ we obtain, see also [134, 135],

$$
\begin{aligned}
F_{2}\left(\mathbf{k}_{1}, \mathbf{k}_{2}\right)=\frac{1}{2} & {\left[k_{i} L_{i}^{(2)}\left(\mathbf{k}_{1}, \mathbf{k}_{2}\right)+k_{i} k_{j} L_{i}^{(1)}\left(\mathbf{k}_{1}\right) L_{j}^{(1)}\left(\mathbf{k}_{2}\right)\right], } \\
F_{3}\left(\mathbf{k}_{1}, \mathbf{k}_{2}, \mathbf{k}_{3}\right)=\frac{1}{3 !} & {\left[k_{i} L_{i}^{(3) s}\left(\mathbf{k}_{1}, \mathbf{k}_{2}, \mathbf{k}_{3}\right)+k_{i} k_{j}\left(L_{i}^{(2)}\left(\mathbf{k}_{1}, \mathbf{k}_{2}\right) L_{j}^{(1)}\left(\mathbf{k}_{3}\right)+\text { cyclic }\right)\right.} \\
& \left.+k_{i} k_{j} k_{k} L_{i}^{(1)}\left(\mathbf{k}_{1}\right) L_{j}^{(1)}\left(\mathbf{k}_{2}\right) L_{k}^{(1)}\left(\mathbf{k}_{3}\right)\right] .
\end{aligned}
$$

Computing the $G_{n}$ kernels is a little more messy than the $F_{n}$, but still straightforward. The velocity field is given by $v_{i}(\mathbf{x}, t)=d \mathbf{x} / d \tau=a \dot{\Psi}_{i}(\mathbf{q}, t)$. We use

$$
\frac{\partial}{\partial q^{i}}=\frac{\partial x^{j}}{\partial q^{i}} \frac{\partial}{\partial x^{j}}=J_{j i} \frac{\partial}{\partial x^{j}},
$$


with $J_{i j}=\partial x^{i} / \partial q^{j}$ the Jacobian matrix of the coordinate transformation (C.1) and $J$ its determinant, to get $\nabla_{\mathbf{x} i}=\left(J^{-1}\right)_{j i} \nabla_{j}$ with $\left(J^{-1}\right)_{j i}=(2 J)^{-1} \epsilon_{i k p} \epsilon_{j q r} J_{k q} J_{p r}$ or

$$
J\left(J^{-1}\right)_{j i}=\delta_{i j}+\left(\delta_{i j} \delta_{a b}-\delta_{i a} \delta_{j b}\right) \Psi_{a, b}+\frac{1}{2} \epsilon_{i k p} \epsilon_{j q r} \Psi_{k, q} \Psi_{p, r} .
$$

Hence

$$
J \frac{1}{a} \nabla_{\mathbf{x} i} v_{i}=\dot{\Psi}_{i, i}+\Psi_{j, j} \dot{\Psi}_{i, i}-\Psi_{i, j} \dot{\Psi}_{i, j}+\frac{1}{2} \epsilon_{i k p} \epsilon_{j q r} \Psi_{k, q} \Psi_{p, r} \dot{\Psi}_{i, j}
$$

The Fourier transform of the velocity divergence yields

$$
\begin{aligned}
-H f \theta(\mathbf{k})= & \int d^{3} x e^{-i \mathbf{k} \cdot \mathbf{x}} \frac{1}{a} \nabla_{\mathbf{x} i} v_{i}=\int d^{3} q e^{-i \mathbf{k} \cdot \mathbf{q}} e^{-i \mathbf{k} \cdot \Psi(\mathbf{q}, t)} J(\mathbf{q}, t) \frac{1}{a} \nabla_{\mathbf{x} i} v_{i} \\
= & \int d^{3} q e^{-i \mathbf{k} \cdot \mathbf{q}} \sum_{\ell=0}^{\infty} \frac{1}{\ell !}\left(-i k_{a} \Psi_{a}\right)^{\ell} \dot{\Psi}_{i, i} \\
& +\left(\delta_{i j} \delta_{a b}-\delta_{i a} \delta_{j b}\right) \int d^{3} q e^{-i \mathbf{k} \cdot \mathbf{q}} \Psi_{a, b} \dot{\Psi}_{i, j} \sum_{\ell=0}^{\infty} \frac{1}{\ell !}\left(-i k_{k} \Psi_{k}\right)^{\ell} \\
& +\frac{1}{2} \epsilon_{i k p} \epsilon_{j q r} \int d^{3} q e^{-i \mathbf{k} \cdot \mathbf{q}} \Psi_{k, q} \Psi_{p, r} \dot{\Psi}_{i, j} \sum_{\ell=0}^{\infty} \frac{1}{\ell !}\left(-i k_{s} \Psi_{s}\right)^{\ell} .
\end{aligned}
$$

On the other hand by taking the derivative of the displacement field

$$
\dot{\Psi}_{i}(\mathbf{p})=i \sum_{m=1}^{\infty} \frac{1}{m !} \int_{\mathbf{p}_{1 \cdots m}=\mathbf{p}} L_{i}^{\prime(m)}\left(\mathbf{p}_{1}, \ldots, \mathbf{p}_{m}\right) \delta_{L}\left(\mathbf{p}_{1}\right) \cdots \delta_{L}\left(\mathbf{p}_{n}\right),
$$

with

$$
L_{i}^{\prime(m)}\left(\mathbf{p}_{1}, \ldots, \mathbf{p}_{m}\right)=\dot{L}_{i}^{(m)}\left(\mathbf{p}_{1}, \ldots, \mathbf{p}_{m}\right)+H L_{i}^{(m)}\left(\mathbf{p}_{1}, \ldots, \mathbf{p}_{m}\right)\left(f\left(\mathbf{p}_{1}\right)+\cdots+f\left(\mathbf{p}_{m}\right)\right) .
$$

- To second order, using eq. (C.9),

$$
\begin{aligned}
-f H \theta^{(2)}(\mathbf{k})= & \int d^{3} q e^{-i \mathbf{k} \cdot \mathbf{q}} \dot{\Psi}_{i, i}^{(2)}(\mathbf{q}, t)-i k_{a} \int d^{3} q e^{-i \mathbf{k} \cdot \mathbf{q}} \Psi_{a}^{(1)}(\mathbf{q}, t) \dot{\Psi}_{i, i}^{(1)}(\mathbf{q}, t) \\
& +\left(\delta_{i j} \delta_{a b}-\delta_{i a} \delta_{j b}\right) \int d^{3} q e^{-i \mathbf{k} \cdot \mathbf{q}} \Psi_{a, b}^{(1)}(\mathbf{q}, t) \dot{\Psi}_{i, j}^{(1)}(\mathbf{q}, t) \\
\equiv & I_{A}+I_{B}+I_{C}
\end{aligned}
$$

with

$$
\begin{aligned}
I_{A}(\mathbf{k}) & =\int d^{3} q e^{-i \mathbf{k} \cdot \mathbf{q}} \nabla_{i} \dot{\Psi}_{i}^{(2)}(\mathbf{q}, t)=\int d^{3} q e^{-i \mathbf{k} \cdot \mathbf{q}} \nabla_{i} \int \frac{d^{3} p}{(2 \pi)^{3}} e^{i \mathbf{p} \cdot \mathbf{q}} \dot{\Psi}_{i}^{(2)}(\mathbf{p}, t) \\
& =\int \frac{d^{3} p}{(2 \pi)^{3}} i \mathbf{p}_{i} \dot{\Psi}_{i}^{(2)}(\mathbf{p}, t) \int d^{3} q e^{i(\mathbf{p}-\mathbf{k}) \cdot \mathbf{q}}=i \mathbf{k}_{i} \dot{\Psi}_{i}^{(2)}(\mathbf{k}, t) \\
& =-\int_{\mathbf{k}_{12}=\mathbf{k}} \frac{1}{2}\left(\mathbf{k}_{1}+\mathbf{k}_{2}\right)_{i} L_{i}^{\prime(2)}\left(\mathbf{k}_{1}, \mathbf{k}_{2}\right) \delta_{1} \delta_{2},
\end{aligned}
$$




$$
\begin{aligned}
I_{B}(\mathbf{k}) & =-\int_{\mathbf{k}_{12}=\mathbf{k}}\left(\mathbf{k}_{1}+\mathbf{k}_{2}\right)_{a} k_{2}^{i} L_{a}^{(1)}\left(\mathbf{k}_{1}\right) L_{i}^{\prime(1)}\left(\mathbf{k}_{2}\right) \delta_{1} \delta_{2} \\
& =-\int_{\mathbf{k}_{12}=\mathbf{k}} \frac{1}{2}\left(\mathbf{k}_{1}+\mathbf{k}_{2}\right)_{a}\left[k_{2}^{i} L_{a}^{(1)}\left(\mathbf{k}_{1}\right) L_{i}^{\prime(1)}\left(\mathbf{k}_{2}\right)+k_{1}^{i} L_{a}^{(1)}\left(\mathbf{k}_{2}\right) L_{i}^{\prime(1)}\left(\mathbf{k}_{1}\right)\right] \delta_{1} \delta_{2} .
\end{aligned}
$$

Now, using

$$
\int d^{3} q e^{-i \mathbf{k} \cdot \mathbf{q}} \Psi_{a, b}^{(1)}(\mathbf{q}) \dot{\Psi}_{i, j}^{(1)}(\mathbf{q})=\int_{\mathbf{k}_{12}=\mathbf{k}} k_{1}^{b} k_{2}^{j} L_{a}^{(1)}\left(\mathbf{k}_{1}\right) L_{i}^{\prime(1)}\left(\mathbf{k}_{2}\right) \delta_{1} \delta_{2},
$$

the last term is

$$
\begin{aligned}
I_{C}(\mathbf{k})= & \left(\delta_{i j} \delta_{a b}-\delta_{i a} \delta_{j b}\right) \int d^{3} q e^{-i \mathbf{k} \cdot \mathbf{q}} \Psi_{a, b}^{(1)}(\mathbf{q}, t) \dot{\Psi}_{i, j}^{(1)}(\mathbf{q}, t) \\
= & \int_{\mathbf{k}_{12}=\mathbf{k}}\left[k_{1}^{a} L_{a}^{(1)}\left(\mathbf{k}_{1}\right) k_{2}^{i} L_{i}^{\prime(1)}\left(\mathbf{k}_{2}\right)-\mathbf{k}_{1} \cdot \mathbf{k}_{2} L_{a}^{(1)}\left(\mathbf{k}_{1}\right) L_{a}^{\prime(1)}\left(\mathbf{k}_{2}\right)\right] \delta_{1} \delta_{2} \\
= & -\int_{\mathbf{k}_{12}=\mathbf{k}} \frac{1}{2}\left[\mathbf{k}_{1} \cdot \mathbf{k}_{2}\left(L_{a}^{(1)}\left(\mathbf{k}_{1}\right) L_{a}^{\prime(1)}\left(\mathbf{k}_{2}\right)+L_{a}^{(1)}\left(\mathbf{k}_{2}\right) L_{a}^{\prime(1)}\left(\mathbf{k}_{1}\right)\right)\right. \\
& \left.\quad-k_{1}^{a} k_{2}^{i} L_{a}^{(1)}\left(\mathbf{k}_{1}\right) L_{i}^{\prime(1)}\left(\mathbf{k}_{2}\right)-k_{2}^{a} k_{1}^{i} L_{a}^{(1)}\left(\mathbf{k}_{2}\right) L_{i}^{\prime(1)}\left(\mathbf{k}_{1}\right)\right] \delta_{1} \delta_{2} .
\end{aligned}
$$

Summing the three contributions and identifying to (2.18), we obtain

$$
\begin{aligned}
2 f H G_{2}\left(\mathbf{k}_{1}, \mathbf{k}_{2}\right) & =\left(\mathbf{k}_{1}+\mathbf{k}_{2}\right)_{i} L_{i}^{\prime(2)}\left(\mathbf{k}_{1}, \mathbf{k}_{2}\right) \\
& +\left(\mathbf{k}_{1}+\mathbf{k}_{2}\right)_{a}\left[k_{2}^{i} L_{a}^{(1)}\left(\mathbf{k}_{1}\right) L_{i}^{\prime(1)}\left(\mathbf{k}_{2}\right)+k_{1}^{i} L_{a}^{(1)}\left(\mathbf{k}_{2}\right) L_{i}^{\prime(1)}\left(\mathbf{k}_{1}\right)\right] \\
& -k_{1}^{a} k_{2}^{i} L_{a}^{(1)}\left(\mathbf{k}_{1}\right) L_{i}^{\prime(1)}\left(\mathbf{k}_{2}\right)-k_{2}^{a} k_{1}^{i} L_{a}^{(1)}\left(\mathbf{k}_{2}\right) L_{i}^{\prime(1)}\left(\mathbf{k}_{1}\right) \\
& +\mathbf{k}_{1} \cdot \mathbf{k}_{2}\left(L_{a}^{(1)}\left(\mathbf{k}_{1}\right) L_{a}^{\prime(1)}\left(\mathbf{k}_{2}\right)+L_{a}^{(1)}\left(\mathbf{k}_{2}\right) L_{a}^{\prime(1)}\left(\mathbf{k}_{1}\right)\right)
\end{aligned}
$$

- To third order, using eq. (C.9),

$$
\begin{aligned}
-f H \theta^{(3)}(\mathbf{k}) & =\int d^{3} q e^{-i \mathbf{k} \cdot \mathbf{q}} \dot{\Psi}_{i, i}^{(3)} \\
& +\int d^{3} q e^{-i \mathbf{k} \cdot \mathbf{q}}\left(-i k_{a} \Psi_{a}^{(1)}\right) \dot{\Psi}_{i, i}^{(2)} \\
& +\int d^{3} q e^{-i \mathbf{k} \cdot \mathbf{q}}\left(-i k_{a} \Psi_{a}^{(2)}\right) \dot{\Psi}_{i, i}^{(1)} \\
& +\frac{1}{2} \int d^{3} q e^{-i \mathbf{k} \cdot \mathbf{q}}\left(-i k_{a} \Psi_{a}^{(1)}\right)\left(-i k_{b} \Psi_{b}^{(1)}\right) \dot{\Psi}_{i, i}^{(1)} \\
& +\left(\delta_{i j} \delta_{a b}-\delta_{i a} \delta_{j b}\right) \int d^{3} q e^{-i \mathbf{k} \cdot \mathbf{q}} \Psi_{a, b}^{(1)} \dot{\Psi}_{i, j}^{(2)} \\
& +\left(\delta_{i j} \delta_{a b}-\delta_{i a} \delta_{j b}\right) \int d^{3} q e^{-i \mathbf{k} \cdot \mathbf{q}} \Psi_{a, b}^{(2)} \dot{\Psi}_{i, j}^{(1)} \\
& +\left(\delta_{i j} \delta_{a b}-\delta_{i a} \delta_{j b}\right) \int d^{3} q e^{-i \mathbf{k} \cdot \mathbf{q}} \Psi_{a, b}^{(1)} \dot{\Psi}_{i, j}^{(1)}\left(-i k_{k} \Psi_{k}^{(1)}\right) \\
& +\frac{1}{2} \epsilon_{i k p} \epsilon_{j q r} \int d^{3} q e^{-i \mathbf{k} \cdot \mathbf{q}} \Psi_{k, q}^{(1)} \Psi_{p, r}^{(1)} \dot{\Psi}_{i, j}^{(1)}
\end{aligned}
$$


The algebra is considerably larger than for the second order case, but it follows the same basic principles from which we arrive to

$$
\begin{aligned}
& G_{3}\left(\mathbf{k}_{1}, \mathbf{k}_{2}, \mathbf{k}_{3}\right)= \\
& \frac{1}{2} C_{3} \Gamma_{3}^{f}\left(\mathbf{k}_{1}, \mathbf{k}_{2}, \mathbf{k}_{3}\right) \\
& +\frac{1}{3} C_{2} \Gamma_{2}^{f}\left(\mathbf{k}_{2}, \mathbf{k}_{3}\right) \frac{\mathbf{k} \cdot \mathbf{k}_{1}}{k_{1}^{2}}+(2 \text { cyclic perm }) \\
& +\frac{1}{6} C_{2} \Gamma_{2}\left(\mathbf{k}_{2}, \mathbf{k}_{3}\right) \frac{\mathbf{k} \cdot \mathbf{k}_{23}}{k_{23}^{2}} \frac{f_{1}}{f_{0}}+(2 \text { cyclic perm }) \\
& +\frac{1}{6} \frac{\left(\mathbf{k} \cdot \mathbf{k}_{1}\right)\left(\mathbf{k} \cdot \mathbf{k}_{2}\right)}{k_{1}^{2} k_{2}^{2}} \frac{f_{3}}{f_{0}}+(2 \text { cyclic perm }) \\
& -\frac{1}{3} C_{2} \Gamma_{2}^{f}\left(\mathbf{k}_{2}, \mathbf{k}_{3}\right)\left(1-\frac{\left(\mathbf{k}_{1} \cdot \mathbf{k}_{23}\right)^{2}}{k_{1}^{2} k_{23}^{2}}\right)+(2 \text { cyclic perm }) \\
& -\frac{1}{6} C_{2} \Gamma_{2}\left(\mathbf{k}_{2}, \mathbf{k}_{3}\right)\left(1-\frac{\left(\mathbf{k}_{1} \cdot \mathbf{k}_{23}\right)^{2}}{k_{1}^{2} k_{23}^{2}}\right) \frac{f_{1}}{f_{0}}+(2 \text { cyclic perm }) \\
& -\frac{1}{3} \frac{\mathbf{k} \cdot \mathbf{k}_{1}}{k_{1}^{2}}\left(1-\frac{\left(\mathbf{k}_{2} \cdot \mathbf{k}_{3}\right)^{2}}{k_{2}^{2} k_{3}^{2}}\right) \frac{f_{2}+f_{3}}{2 f_{0}}+(2 \text { cyclic perm }) \\
& +3\left[1-\frac{\left(\mathbf{k}_{1} \cdot \mathbf{k}_{2}\right)^{2}}{k_{1}^{2} k_{2}^{2}}-\frac{\left(\mathbf{k}_{2} \cdot \mathbf{k}_{3}\right)^{2}}{k_{2}^{2} k_{3}^{2}}-\frac{\left(\mathbf{k}_{3} \cdot \mathbf{k}_{1}\right)^{2}}{k_{3}^{2} k_{1}^{2}}\right. \\
& \left.\quad+2 \frac{\left(\mathbf{k}_{1} \cdot \mathbf{k}_{2}\right)\left(\mathbf{k}_{2} \cdot \mathbf{k}_{3}\right)\left(\mathbf{k}_{3} \cdot \mathbf{k}_{1}\right)}{k_{1}^{2} k_{2}^{2} k_{3}^{2}}\right] \frac{f_{1}+f_{2}+f_{3}}{3 f_{0}} .
\end{aligned}
$$

We have used the scalar kernels $C_{n} \Gamma_{n}$ for the transverse piece of the Lagrangian displacement, $\mathbf{k} \cdot \Psi$, defined as

$$
\begin{aligned}
& C_{n} \Gamma_{n}\left(\mathbf{k}_{1}, \ldots, \mathbf{k}_{n}\right)=k_{1 \cdots n}^{i} L_{i}^{(n)}\left(\mathbf{k}_{1}, \ldots, \mathbf{k}_{n}\right), \\
& C_{n} \Gamma_{n}^{f}\left(\mathbf{k}_{1}, \ldots, \mathbf{k}_{n}\right)=\frac{1}{n H f_{0}} k_{1 \cdots n}^{i} L_{i}^{\prime(n)}\left(\mathbf{k}_{1}, \ldots, \mathbf{k}_{n}\right),
\end{aligned}
$$

with $C_{n}$ a set of numbers, from which the first two are fixed to $C_{1}=1$ and $C_{2}=3 / 7$ for simplicity. Since for 1-loop, 2-point statistics, the transverse component of Lagrangian displacements project out, one can use the scalar $\Gamma$ instead of vector $\mathbf{L}$ kernels without loss of generality.

We have related the second and third order SPT kernels to the LPT kernels and their derivatives through eqs. (C.4), (C.5), (C.20) and (C.22). We take the LPT kernels from ref. [29]. For $F_{2}$ and $G_{2}$ we arrive at the expressions of eqs. (2.20) and (2.21).

For the third order kernels $F_{3}, G_{3}\left(\mathbf{k}_{1}, \mathbf{k}_{2}, \mathbf{k}_{3}\right)$ we are interested in double-squeezed configurations, on which $\mathbf{k}_{1}=\mathbf{k}$ and $\mathbf{k}_{3}=-\mathbf{k}_{2}=\mathbf{p}$. To write the final expressions for these kernels we consider the first order scalar kernels, that are $\Gamma_{1}(\mathbf{k})=1$ and $\Gamma_{1}^{f}(\mathbf{k})=f(k) / f_{0}$. The second order scalar kernels are

$$
\begin{aligned}
\Gamma_{2}\left(\mathbf{p}_{1}, \mathbf{p}_{2}\right) & =\left[\mathcal{A}\left(\mathbf{p}_{1}, \mathbf{p}_{2}\right)-\mathcal{B}\left(\mathbf{p}_{1}, \mathbf{p}_{2}\right) \frac{\left(\mathbf{p}_{1} \cdot \mathbf{p}_{2}\right)^{2}}{p_{1}^{2} p_{2}^{2}}\right], \\
\Gamma_{2}^{f}\left(\mathbf{p}_{1}, \mathbf{p}_{2}\right) & =\Gamma_{2}\left(\mathbf{p}_{1}, \mathbf{p}_{2}\right) \frac{f\left(p_{1}\right)+f\left(p_{2}\right)}{2 f_{0}}+\frac{1}{2 f_{0} H}\left[\dot{\mathcal{A}}\left(\mathbf{p}_{1}, \mathbf{p}_{2}\right)-\dot{\mathcal{B}}\left(\mathbf{p}_{1}, \mathbf{p}_{2}\right) \frac{\left(\mathbf{p}_{1} \cdot \mathbf{p}_{2}\right)^{2}}{p_{1}^{2} p_{2}^{2}}\right],
\end{aligned}
$$


where $\mathcal{A}, \mathcal{B}=\mathcal{A}, \mathcal{B}\left(\mathbf{p}_{1}, \mathbf{p}_{2}\right)$ are the second order growth functions defined by eqs. (2.22). The third order kernels are

$$
\begin{aligned}
C_{3} \Gamma_{3}\left[\mathbf{p}_{1}, \mathbf{p}_{2}, \mathbf{p}_{3}\right] & =\frac{D_{+}^{(3) s}\left(\mathbf{p}_{1}, \mathbf{p}_{2}, \mathbf{p}_{3}\right)}{D_{+}\left(\mathbf{p}_{1}\right) D_{+}\left(\mathbf{p}_{2}\right) D_{+}\left(\mathbf{p}_{3}\right)}, \\
C_{3} \Gamma_{3}^{f}\left[\mathbf{p}_{1}, \mathbf{p}_{2}, \mathbf{p}_{3}\right] & =\frac{1}{3 f_{0}} \frac{\frac{d}{d \ln a} D_{+}^{(3) s}\left(\mathbf{p}_{1}, \mathbf{p}_{2}, \mathbf{p}_{3}\right)}{D_{+}\left(\mathbf{p}_{1}\right) D_{+}\left(\mathbf{p}_{2}\right) D_{+}\left(\mathbf{p}_{3}\right)} .
\end{aligned}
$$

with the (symmetric) third order growth function $D_{+}^{(3) s}$ as given in [29]. ${ }^{12}$ Actually, we will not use the value of $C_{3}$ at all, so we can let it free. But by defining $C_{2}=3 / 7$ we make the notation simpler.

The third order kernels are

$$
\begin{gathered}
F_{3}(\mathbf{k},-\mathbf{p}, \mathbf{p})=\frac{1}{6} C_{3} \Gamma_{3}(\mathbf{k},-\mathbf{p}, \mathbf{p})+\frac{1}{3} C_{2} \frac{\mathbf{k} \cdot(\mathbf{k}-\mathbf{p}) \mathbf{k} \cdot \mathbf{p}}{p^{2}|\mathbf{k}-\mathbf{p}|^{2}} \Gamma_{2}(\mathbf{k},-\mathbf{p})-\frac{1}{6} \frac{(\mathbf{k} \cdot \mathbf{p})^{2}}{p^{4}} \\
G_{3}(\mathbf{k},-\mathbf{p}, \mathbf{p})=\frac{1}{2} C_{3} \Gamma_{3}^{f}(\mathbf{k},-\mathbf{p}, \mathbf{p})+\frac{2}{3} C_{2} \frac{\mathbf{k} \cdot \mathbf{p}}{p^{2}} \Gamma_{2}^{f}(\mathbf{k},-\mathbf{p})+\frac{1}{3} C_{2} \frac{f(p)}{f_{0}} \Gamma_{2}(\mathbf{k},-\mathbf{p}) \frac{\mathbf{k} \cdot(\mathbf{k}-\mathbf{p})}{|\mathbf{k}-\mathbf{p}|^{2}} \\
-\frac{1}{6} \frac{(\mathbf{k} \cdot \mathbf{p})^{2}}{p^{4}} \frac{f(k)}{f_{0}}-\frac{1}{3} C_{2}\left[2 \Gamma_{2}^{f}(\mathbf{k},-\mathbf{p})+\Gamma(\mathbf{k},-\mathbf{p}) \frac{f(p)}{f_{0}}\right]\left[1-\frac{(\mathbf{p} \cdot(\mathbf{k}-\mathbf{p}))^{2}}{p^{2}|\mathbf{k}-\mathbf{p}|^{2}}\right],
\end{gathered}
$$

which are valid upon integration with $d^{3} p$. It is worth noticing that the second term in the second line yields zero in $\Lambda$ CDM because $\int_{-1}^{1} d x x\left(1-x^{2}\right)=0$. But in general cosmologies do not because of the $x$ dependence of functions $\mathcal{A}$ and $\mathcal{B}$. Notoriously, for DGP or cubic Galileons, $\mathcal{B}$ is only time dependent and the dependence on $x$ of $\mathcal{A}$ contains only zero and second Legendre Polynomials, such that $x\left(\mathcal{A}-\mathcal{B} x^{2}\right)$ is odd in $x$ and hence that term is also zero.

We notice that the approximation of static kernels, usually taken in $\Lambda \mathrm{CDM}$ and exact for EdS, corresponds to

$$
\Gamma_{n}^{f} \simeq \Gamma_{n}, \quad(\Lambda \mathrm{CDM})
$$

\section{References}

[1] 2DFGRS collaboration, S. Cole et al., The 2dF Galaxy Redshift Survey: Power-spectrum analysis of the final dataset and cosmological implications, Mon. Not. Roy. Astron. Soc. 362 (2005) 505-534, [astro-ph/0501174].

[2] C. Blake et al., The WiggleZ Dark Energy Survey: mapping the distance-redshift relation with baryon acoustic oscillations, Mon. Not. Roy. Astron. Soc. 418 (2011) 1707-1724, [1108.2635].

[3] L. Samushia et al., The clustering of galaxies in the SDSS-III Baryon Oscillation Spectroscopic Survey: measuring growth rate and geometry with anisotropic clustering, Mon. Not. Roy. Astron. Soc. 439 (2014) 3504-3519, [1312.4899].

[4] Z. Zhai et al., The Clustering of Luminous Red Galaxies at $z \sim 0.7$ from EBOSS and BOSS Data, Astrophys. J. 848 (2017) 76, [1607.05383].

[5] DES collaboration, T. Abbott et al., Dark Energy Survey year 1 results: Cosmological constraints from galaxy clustering and weak lensing, Phys. Rev. D 98 (2018) 043526, [1708.01530].

\footnotetext{
${ }^{12}$ After correcting a trivial typo, as in the expressions of appendix of [31], or eq. (A5) of [123].
} 
[6] DESI collaboration, A. Aghamousa et al., The DESI Experiment Part I: Science,Targeting, and Survey Design, 1611.00036.

[7] LSST Dark Energy Science collaboration, A. Abate et al., Large Synoptic Survey Telescope: Dark Energy Science Collaboration, 1211.0310.

[8] Euclid collaboration, R. Scaramella et al., Euclid space mission: a cosmological challenge for the next 15 years, IAU Symp. 306 (2014) 375-378, [1501.04908].

[9] N. Kaiser, Clustering in real space and in redshift space, Monthly Notices of the Royal Astronomical Society 227 (07, 1987) 1-21.

[10] J. A. Peacock et al., A Measurement of the cosmological mass density from clustering in the 2dF Galaxy Redshift Survey, Nature 410 (2001) 169-173, [astro-ph/0103143].

[11] E. Hawkins et al., The 2dF Galaxy Redshift Survey: Correlation functions, peculiar velocities and the matter density of the universe, Mon. Not. Roy. Astron. Soc. 346 (2003) 78, [astro-ph/0212375].

[12] SDSS collaboration, M. Tegmark et al., Cosmological Constraints from the SDSS Luminous Red Galaxies, Phys. Rev. D 74 (2006) 123507, [astro-ph/0608632].

[13] C. Alcock and B. Paczynski, An evolution free test for non-zero cosmological constant, Nature 281 (Oct, 1979) 358-359.

[14] E. Hivon, F. Bouchet, S. Colombi and R. Juszkiewicz, Redshift distortions of clustering: A Lagrangian approach, Astron. Astrophys. 298 (1995) 643-660, [astro-ph/9407049].

[15] K. Fisher and A. Nusser, The nonlinear redshift space power spectrum: omega from redshift surveys, Mon. Not. Roy. Astron. Soc. 279 (1996) L1, [astro-ph/9510049].

[16] A. Taylor and A. Hamilton, Nonlinear cosmological power spectra in real and redshift space, Mon. Not. Roy. Astron. Soc. 282 (1996) 767, [astro-ph/9604020].

[17] A. Heavens, S. Matarrese and L. Verde, The Nonlinear redshift-space power spectrum of galaxies, Mon. Not. Roy. Astron. Soc. 301 (1998) 797-808, [astro-ph/9808016].

[18] H. Magira, Y. Jing and Y. Suto, Cosmological redshift-space distortion on clustering of high-redshift objects: correction for nonlinear effects in power spectrum and tests with n-body simulations, Astrophys. J. 528 (2000) 30, [astro-ph/9907438].

[19] R. Scoccimarro, Redshift-space distortions, pairwise velocities and nonlinearities, Phys. Rev. D 70 (2004) 083007, [astro-ph/0407214].

[20] T. Matsubara, Nonlinear perturbation theory with halo bias and redshift-space distortions via the Lagrangian picture, Phys. Rev. D 78 (2008) 083519, [0807.1733].

[21] W. J. Percival and M. White, Testing cosmological structure formation using redshift-space distortions, Mon. Not. Roy. Astron. Soc. 393 (2009) 297, [0808.0003].

[22] A. Taruya, T. Nishimichi and S. Saito, Baryon Acoustic Oscillations in 2D: Modeling Redshift-space Power Spectrum from Perturbation Theory, Phys. Rev. D82 (2010) 063522, [1006.0699].

[23] F. Bernardeau, S. Colombi, E. Gaztanaga and R. Scoccimarro, Large scale structure of the universe and cosmological perturbation theory, Phys. Rept. 367 (2002) 1-248, [astro-ph/0112551].

[24] L. Samushia et al., The Clustering of Galaxies in the SDSS-III DR9 Baryon Oscillation Spectroscopic Survey: Testing Deviations from $\Lambda$ and General Relativity using anisotropic clustering of galaxies, Mon. Not. Roy. Astron. Soc. 429 (2013) 1514-1528, [1206. 5309].

[25] E. Macaulay, I. K. Wehus and H. K. Eriksen, Lower Growth Rate from Recent Redshift Space Distortion Measurements than Expected from Planck, Phys. Rev. Lett. 111 (2013) 161301, [1303.6583]. 
[26] BOSS collaboration, F. Beutler et al., The clustering of galaxies in the SDSS-III Baryon Oscillation Spectroscopic Survey: Testing gravity with redshift-space distortions using the power spectrum multipoles, Mon. Not. Roy. Astron. Soc. 443 (2014) 1065-1089, [1312.4611].

[27] BOSS collaboration, F. Beutler et al., The clustering of galaxies in the completed SDSS-III Baryon Oscillation Spectroscopic Survey: Anisotropic galaxy clustering in Fourier-space, Mon. Not. Roy. Astron. Soc. 466 (2017) 2242-2260, [1607.03150].

[28] K. Koyama, A. Taruya and T. Hiramatsu, Non-linear Evolution of Matter Power Spectrum in Modified Theory of Gravity, Phys. Rev. D 79 (2009) 123512, [0902.0618].

[29] A. Aviles and J. L. Cervantes-Cota, Lagrangian perturbation theory for modified gravity, Phys. Rev. D96 (2017) 123526, [1705.10719].

[30] A. Taruya, Constructing perturbation theory kernels for large-scale structure in generalized cosmologies, Phys. Rev. D94 (2016) 023504, [1606.02168].

[31] A. Aviles, J. L. Cervantes-Cota and D. F. Mota, Screenings in Modified Gravity: a perturbative approach, Astron. Astrophys. 622 (2019) A62, [1810.02652].

[32] B. Li, G.-B. Zhao, R. Teyssier and K. Koyama, ECOSMOG: An Efficient Code for Simulating Modified Gravity, JCAP 01 (2012) 051, [1110.1379].

[33] M. Cataneo, L. Lombriser, C. Heymans, A. Mead, A. Barreira, S. Bose et al., On the road to percent accuracy: non-linear reaction of the matter power spectrum to dark energy and modified gravity, Mon. Not. Roy. Astron. Soc. 488 (2019) 2121-2142, [1812.05594].

[34] G. Valogiannis and R. Bean, Efficient simulations of large scale structure in modified gravity cosmologies with comoving Lagrangian acceleration, Phys. Rev. D95 (2017) 103515, [1612.06469].

[35] H. A. Winther, K. Koyama, M. Manera, B. S. Wright and G.-B. Zhao, COLA with scale-dependent growth: applications to screened modified gravity models, JCAP 08 (2017) 006, [1703.00879].

[36] S. Alam et al., Testing the theory of gravity with DESI: estimators, predictions and simulation requirements, 2011.05771.

[37] E. V. Linder, Redshift Distortions as a Probe of Gravity, Astropart. Phys. 29 (2008) 336-339, [0709.1113].

[38] Y. Wang, Differentiating dark energy and modified gravity with galaxy redshift surveys, JCAP 05 (2008) 021, [0710.3885].

[39] L. Guzzo et al., A test of the nature of cosmic acceleration using galaxy redshift distortions, Nature 451 (2008) 541-545, [0802.1944].

[40] K. Yamamoto, T. Sato and G. Huetsi, Testing general relativity with the multipole spectra of the SDSS luminous red galaxies, Prog. Theor. Phys. 120 (2008) 609-614, [0805.4789].

[41] F. Simpson and J. A. Peacock, Difficulties Distinguishing Dark Energy from Modified Gravity via Redshift Distortions, Phys. Rev. D 81 (2010) 043512, [0910.3834].

[42] A. Stril, R. N. Cahn and E. V. Linder, Testing Standard Cosmology with Large Scale Structure, Mon. Not. Roy. Astron. Soc. 404 (2010) 239, [0910.1833].

[43] R. Bean and M. Tangmatitham, Current constraints on the cosmic growth history, Phys. Rev. D 81 (2010) 083534, [1002.4197].

[44] Y.-S. Song and W. J. Percival, Reconstructing the history of structure formation using Redshift Distortions, JCAP 10 (2009) 004, [0807.0810].

[45] J. Guzik, B. Jain and M. Takada, Tests of Gravity from Imaging and Spectroscopic Surveys, Phys. Rev. D 81 (2010) 023503, [0906.2221]. 
[46] Y.-S. Song, G.-B. Zhao, D. Bacon, K. Koyama, R. C. Nichol and L. Pogosian, Complementarity of Weak Lensing and Peculiar Velocity Measurements in Testing General Relativity, Phys. Rev. D 84 (2011) 083523, [1011.2106].

[47] S. Asaba, C. Hikage, K. Koyama, G.-B. Zhao, A. Hojjati and L. Pogosian, Principal Component Analysis of Modified Gravity using Weak Lensing and Peculiar Velocity Measurements, JCAP 08 (2013) 029, [1306.2546].

[48] A. Taruya, K. Koyama, T. Hiramatsu and A. Oka, Beyond consistency test of gravity with redshift-space distortions at quasilinear scales, Phys. Rev. D 89 (2014) 043509, [1309.6783].

[49] A. Taruya, T. Nishimichi, F. Bernardeau, T. Hiramatsu and K. Koyama, Regularized cosmological power spectrum and correlation function in modified gravity models, Phys. Rev. D 90 (2014) 123515, [1408.4232].

[50] B. Bose and K. Koyama, A Perturbative Approach to the Redshift Space Correlation Function: Beyond the Standard Model, JCAP 08 (2017) 029, [1705.09181].

[51] G. Valogiannis, R. Bean and A. Aviles, An accurate perturbative approach to redshift space clustering of biased tracers in modified gravity, JCAP 01 (2020) 055, [1909.05261].

[52] B. Bose, H. A. Winther, A. Pourtsidou, S. Casas, L. Lombriser, Q. Xia et al., Hybrid $P_{\ell}(k)$ : general, unified, non-linear matter power spectrum in redshift space, JCAP 09 (2020) 001, [1911.04391].

[53] H. Oyaizu, Non-linear evolution of $f(R)$ cosmologies I: methodology, Phys. Rev. D 78 (2008) 123523, [0807.2449].

[54] H. Oyaizu, M. Lima and W. Hu, Nonlinear evolution of $f(R)$ cosmologies. 2. Power spectrum, Phys. Rev. D 78 (2008) 123524, [0807.2462].

[55] F. Schmidt, M. V. Lima, H. Oyaizu and W. Hu, Non-linear Evolution of $f(R)$ Cosmologies III: Halo Statistics, Phys. Rev. D 79 (2009) 083518, [0812.0545].

[56] F. Schmidt, Self-Consistent Cosmological Simulations of DGP Braneworld Gravity, Phys. Rev. D 80 (2009) 043001, [0905.0858].

[57] G.-B. Zhao, B. Li and K. Koyama, N-body Simulations for $f(R)$ Gravity using a Self-adaptive Particle-Mesh Code, Phys. Rev. D 83 (2011) 044007, [1011.1257].

[58] B. Li and H. Zhao, Structure Formation by Fifth Force I: N-Body vs. Linear Simulations, Phys. Rev. D 80 (2009) 044027, [0906.3880].

[59] B. Li and H. Zhao, Structure Formation by the Fifth Force III: Segregation of Baryons and Dark Matter, Phys. Rev. D 81 (2010) 104047, [1001.3152].

[60] B. Li and J. D. Barrow, N-Body Simulations for Coupled Scalar Field Cosmology, Phys. Rev. D 83 (2011) 024007, [1005.4231].

[61] B. Li, W. A. Hellwing, K. Koyama, G.-B. Zhao, E. Jennings and C. M. Baugh, The nonlinear matter and velocity power spectra in $f(R)$ gravity, Mon. Not. Roy. Astron. Soc. 428 (2013) $743-755,[1206.4317]$.

[62] E. Jennings, C. M. Baugh, B. Li, G.-B. Zhao and K. Koyama, Redshift space distortions in f(R) gravity, Mon. Not. Roy. Astron. Soc. 425 (2012) 2128-2143, [1205.2698].

[63] P. Arnalte-Mur, W. A. Hellwing and P. Norberg, Real- and redshift-space halo clustering in $f(R)$ cosmologies, Mon. Not. Roy. Astron. Soc. 467 (2017) 1569-1585, [1612. 02355].

[64] C. Hernández-Aguayo, J. Hou, B. Li, C. M. Baugh and A. G. Sánchez, Large-scale redshift space distortions in modified gravity theories, Mon. Not. Roy. Astron. Soc. 485 (2019) 2194-2213, [1811.09197]. 
[65] Y.-S. Song, A. Taruya, E. Linder, K. Koyama, C. G. Sabiu, G.-B. Zhao et al., Consistent Modified Gravity Analysis of Anisotropic Galaxy Clustering Using BOSS DR11, Phys. Rev. D 92 (2015) 043522, [1507.01592].

[66] J. Dossett, B. Hu and D. Parkinson, Constraining models of $f(R)$ gravity with Planck and WiggleZ power spectrum data, JCAP 03 (2014) 046, [1401.3980].

[67] A. Johnson, C. Blake, J. Dossett, J. Koda, D. Parkinson and S. Joudaki, Searching for Modified Gravity: Scale and Redshift Dependent Constraints from Galaxy Peculiar Velocities, Mon. Not. Roy. Astron. Soc. 458 (2016) 2725-2744, [1504.06885].

[68] J.-h. He, L. Guzzo, B. Li and C. M. Baugh, No evidence for modifications of gravity from galaxy motions on cosmological scales, Nature Astron. 2 (2018) 967-972, [1809.09019].

[69] A. Taruya, T. Nishimichi and F. Bernardeau, Precision modeling of redshift-space distortions from a multipoint propagator expansion, Phys. Rev. D 87 (2013) 083509, [1301.3624].

[70] A. Barreira, A. G. Sánchez and F. Schmidt, Validating estimates of the growth rate of structure with modified gravity simulations, Phys. Rev. D 94 (2016) 084022, [1605.03965].

[71] B. Bose, K. Koyama, W. A. Hellwing, G.-B. Zhao and H. A. Winther, Theoretical accuracy in cosmological growth estimation, Phys. Rev. D 96 (2017) 023519, [1702.02348].

[72] Z. Vlah and M. White, Exploring redshift-space distortions in large-scale structure, JCAP 1903 (2019) 007, [1812.02775].

[73] S.-F. Chen, Z. Vlah and M. White, Consistent Modeling of Velocity Statistics and Redshift-Space Distortions in One-Loop Perturbation Theory, JCAP 07 (2020) 062, [2005.00523].

[74] M. M. Ivanov, M. Simonović and M. Zaldarriaga, Cosmological Parameters from the BOSS Galaxy Power Spectrum, JCAP 05 (2020) 042, [1909.05277].

[75] M. M. Ivanov and S. Sibiryakov, Infrared Resummation for Biased Tracers in Redshift Space, JCAP 07 (2018) 053, [1804.05080].

[76] A. Chudaykin, M. M. Ivanov, O. H. Philcox and M. Simonović, Nonlinear perturbation theory extension of the Boltzmann code CLASS, Phys. Rev. D 102 (2020) 063533, [2004.10607].

[77] P. McDonald and A. Roy, Clustering of dark matter tracers: generalizing bias for the coming era of precision LSS, JCAP 0908 (2009) 020, [0902.0991].

[78] S. Saito, T. Baldauf, Z. Vlah, U. Seljak, T. Okumura and P. McDonald, Understanding higher-order nonlocal halo bias at large scales by combining the power spectrum with the bispectrum, Phys. Rev. D90 (2014) 123522, [1405.1447].

[79] M. Cautun, E. Paillas, Y.-C. Cai, S. Bose, J. Armijo, B. Li et al., The Santiago-Harvard-Edinburgh-Durham void comparison - I. SHEDding light on chameleon gravity tests, Mon. Not. Roy. Astron. Soc. 476 (2018) 3195-3217, [1710.01730].

[80] P. Brax and P. Valageas, Impact on the power spectrum of Screening in Modified Gravity Scenarios, Phys. Rev. D 88 (2013) 023527, [1305.5647].

[81] A. Aviles and A. Banerjee, A Lagrangian Perturbation Theory in the presence of massive neutrinos, JCAP 10 (2020) 034, [2007.06508].

[82] T. Matsubara, Recursive Solutions of Lagrangian Perturbation Theory, Phys. Rev. D 92 (2015) 023534, [1505.01481].

[83] A. Aviles, M. A. Rodriguez-Meza, J. De-Santiago and J. L. Cervantes-Cota, Nonlinear evolution of initially biased tracers in modified gravity, JCAP 1811 (2018) 013, [1809.07713].

[84] P. McDonald, Clustering of dark matter tracers: Renormalizing the bias parameters, Phys. Rev. D 74 (2006) 103512, [astro-ph/0609413]. 
[85] L. Hui and K. P. Parfrey, The Evolution of Bias: Generalized, Phys. Rev. D77 (2008) 043527, [0712.1162].

[86] F. Villaescusa-Navarro, F. Marulli, M. Viel, E. Branchini, E. Castorina, E. Sefusatti et al., Cosmology with massive neutrinos I: towards a realistic modeling of the relation between matter, haloes and galaxies, JCAP 03 (2014) 011, [1311.0866].

[87] E. Castorina, E. Sefusatti, R. K. Sheth, F. Villaescusa-Navarro and M. Viel, Cosmology with massive neutrinos II: on the universality of the halo mass function and bias, JCAP 02 (2014) 049, [1311.1212].

[88] M. LoVerde, Halo bias in mixed dark matter cosmologies, Phys. Rev. D 90 (2014) 083530, [1405.4855].

[89] S. Vagnozzi, T. Brinckmann, M. Archidiacono, K. Freese, M. Gerbino, J. Lesgourgues et al., Bias due to neutrinos must not uncorrect'd go, JCAP 09 (2018) 001, [1807.04672].

[90] A. Banerjee, E. Castorina, F. Villaescusa-Navarro, T. Court and M. Viel, Weighing neutrinos with the halo environment, JCAP 06 (2020) 032, [1907.06598].

[91] V. Desjacques, D. Jeong and F. Schmidt, Large-Scale Galaxy Bias, Phys. Rept. 733 (2018) 1-193, [1611.09787].

[92] V. Desjacques, D. Jeong and F. Schmidt, The Galaxy Power Spectrum and Bispectrum in Redshift Space, JCAP 12 (2018) 035, [1806.04015].

[93] Y. Donath and L. Senatore, Biased Tracers in Redshift Space in the EFTofLSS with exact time dependence, JCAP 10 (2020) 039, [2005.04805].

[94] M. Jalilvand, B. Ghosh, E. Majerotto, B. Bose, R. Durrer and M. Kunz, Nonlinear contributions to angular power spectra, Phys. Rev. D 101 (2020) 043530, [1907.13109].

[95] A. Perko, L. Senatore, E. Jennings and R. H. Wechsler, Biased Tracers in Redshift Space in the EFT of Large-Scale Structure, 1610.09321.

[96] O. H. Philcox, A. Aviles and E. Massara, Modeling the Marked Spectrum of Matter and Biased Tracers in Real- and Redshift-Space, 2010.05914.

[97] D. Baumann, A. Nicolis, L. Senatore and M. Zaldarriaga, Cosmological Non-Linearities as an Effective Fluid, JCAP 07 (2012) 051, [1004.2488].

[98] M. P. Hertzberg, Effective field theory of dark matter and structure formation: Semianalytical results, Phys. Rev. D 89 (2014) 043521, [1208.0839].

[99] G. D'Amico, J. Gleyzes, N. Kokron, K. Markovic, L. Senatore, P. Zhang et al., The Cosmological Analysis of the SDSS/BOSS data from the Effective Field Theory of Large-Scale Structure, JCAP 05 (2020) 005, [1909.05271].

[100] T. Nishimichi, G. D’Amico, M. M. Ivanov, L. Senatore, M. Simonović, M. Takada et al., Blinded challenge for precision cosmology with large-scale structure: results from effective field theory for the redshift-space galaxy power spectrum, 2003.08277.

[101] M. Schmittfull, M. Simonović, M. M. Ivanov, O. H. E. Philcox and M. Zaldarriaga, Modeling Galaxies in Redshift Space at the Field Level, 2012.03334.

[102] T. Colas, G. D'amico, L. Senatore, P. Zhang and F. Beutler, Efficient Cosmological Analysis of the SDSS/BOSS data from the Effective Field Theory of Large-Scale Structure, JCAP 06 (2020) 001, [1909.07951].

[103] D. J. Eisenstein, H.-j. Seo, E. Sirko and D. Spergel, Improving Cosmological Distance Measurements by Reconstruction of the Baryon Acoustic Peak, Astrophys. J. 664 (2007) 675-679, [astro-ph/0604362].

[104] M. Crocce and R. Scoccimarro, Nonlinear Evolution of Baryon Acoustic Oscillations, Phys. Rev. D 77 (2008) 023533, [0704.2783]. 
[105] J. Carlson, B. Reid and M. White, Convolution lagrangian perturbation theory for biased tracers, Monthly Notices of the Royal Astronomical Society 429 (Dec, 2012) 1674-1685.

[106] A. Taruya, F. Bernardeau, T. Nishimichi and S. Codis, Direct and fast calculation of regularized cosmological power spectrum at two-loop order, Phys. Rev. D 86 (Nov, 2012) 103528.

[107] L. Senatore and M. Zaldarriaga, The IR-resummed Effective Field Theory of Large Scale Structures, JCAP 02 (2015) 013, [1404.5954].

[108] T. Baldauf, M. Mirbabayi, M. Simonović and M. Zaldarriaga, Equivalence Principle and the Baryon Acoustic Peak, Phys. Rev. D 92 (2015) 043514, [1504.04366].

[109] M. Peloso and M. Pietroni, Galilean invariant resummation schemes of cosmological perturbations, JCAP 01 (2017) 056, [1609.06624].

[110] Z. Vlah, M. White and A. Aviles, A Lagrangian effective field theory, JCAP 09 (2015) 014, [1506.05264].

[111] Z. Vlah, U. Seljak, M. Y. Chu and Y. Feng, Perturbation theory, effective field theory, and oscillations in the power spectrum, JCAP 03 (2016) 057, [1509.02120].

[112] D. Blas, M. Garny, M. M. Ivanov and S. Sibiryakov, Time-Sliced Perturbation Theory II: Baryon Acoustic Oscillations and Infrared Resummation, JCAP 07 (2016) 028, [1605.02149].

[113] Z. Ding, H.-J. Seo, Z. Vlah, Y. Feng, M. Schmittfull and F. Beutler, Theoretical Systematics of Future Baryon Acoustic Oscillation Surveys, Mon. Not. Roy. Astron. Soc. 479 (2018) 1021-1054, [1708.01297].

[114] L. Senatore and G. Trevisan, On the IR-Resummation in the EFTofLSS, JCAP 05 (2018) 019, [1710.02178].

[115] M. Lewandowski and L. Senatore, An analytic implementation of the IR-resummation for the BAO peak, JCAP 03 (2020) 018, [1810.11855].

[116] J. Hamann, S. Hannestad, J. Lesgourgues, C. Rampf and Y. Y. Wong, Cosmological parameters from large scale structure - geometric versus shape information, JCAP 07 (2010) 022, [1003.3999].

[117] S.-F. Chen, Z. Vlah, E. Castorina and M. White, Redshift-Space Distortions in Lagrangian Perturbation Theory, 2012.04636.

[118] G. Dvali, G. Gabadadze and M. Porrati, 4-D gravity on a brane in 5-D Minkowski space, Phys. Lett. B 485 (2000) 208-214, [hep-th/0005016].

[119] A. Nicolis, R. Rattazzi and E. Trincherini, The Galileon as a local modification of gravity, Phys. Rev. D 79 (2009) 064036, [0811.2197].

[120] B. Li, G.-B. Zhao, R. Teyssier and K. Koyama, Ecosmog : an efficient code for simulating modified gravity, Journal of Cosmology and Astroparticle Physics 2012 (2012) 051.

[121] S. Bose, B. Li, A. Barreira, J.-h. He, W. A. Hellwing, K. Koyama et al., Speeding up N-body simulations of modified gravity: Chameleon screening models, JCAP 1702 (2017) 050, [1611.09375].

[122] P. S. Behroozi, R. H. Wechsler and H.-Y. Wu, The ROCKSTAR Phase-space Temporal Halo Finder and the Velocity Offsets of Cluster Cores, The Astrophysical Journal 762 (Jan., 2013) $109,[1110.4372]$.

[123] G. Valogiannis and R. Bean, Convolution Lagrangian perturbation theory for biased tracers beyond general relativity, Phys. Rev. D 99 (2019) 063526, [1901.03763].

[124] L. M. Fernández-Hernández, A. Montiel and M. A. Rodríguez-Meza, Galaxy rotation curves using a non-parametric regression method: core, cuspy and fuzzy scalar field dark matter models, MNRAS 488 (Oct., 2019) 5127-5144, [1809.06875]. 
[125] B. A. Reid and M. White, Towards an accurate model of the redshift space clustering of halos in the quasilinear regime, Mon. Not. Roy. Astron. Soc. 417 (2011) 1913-1927, [1105.4165].

[126] A. Lewis, A. Challinor and A. Lasenby, Efficient computation of CMB anisotropies in closed FRW models, ApJ 538 (2000) 473-476, [astro-ph/9911177].

[127] B. Giblin, M. Cataneo, B. Moews and C. Heymans, On the road to per cent accuracy - II. Calibration of the non-linear matter power spectrum for arbitrary cosmologies, Mon. Not. Roy. Astron. Soc. 490 (2019) 4826-4840, [1906.02742].

[128] LSST Dark Energy Science collaboration, N. Ramachandra, G. Valogiannis, M. Ishak and K. Heitmann, Matter Power Spectrum Emulator for f(R) Modified Gravity Cosmologies, 2010.00596.

[129] M. Schmittfull, Z. Vlah and P. McDonald, Fast large scale structure perturbation theory using one-dimensional fast Fourier transforms, Phys. Rev. D 93 (2016) 103528, [1603.04405].

[130] J. E. McEwen, X. Fang, C. M. Hirata and J. A. Blazek, FAST-PT: a novel algorithm to calculate convolution integrals in cosmological perturbation theory, JCAP 09 (2016) 015, [1603.04826].

[131] R. Liu, G. Valogiannis, N. Battaglia and R. Bean, Constraints on $f(R)$ and nDGP Modified Gravity Model Parameters with Cluster Abundances and Galaxy Clustering, 2101.08728.

[132] "Digital library of mathematica functions (dlmf)." https://dlmf.nist.gov/16.

[133] W. Hu and I. Sawicki, Models of $f(R)$ Cosmic Acceleration that Evade Solar-System Tests, Phys. Rev. D 76 (2007) 064004, [0705.1158].

[134] T. Matsubara, Nonlinear Perturbation Theory Integrated with Nonlocal Bias, Redshift-space Distortions, and Primordial Non-Gaussianity, Phys. Rev. D 83 (2011) 083518, [1102.4619].

[135] C. Rampf and T. Buchert, Lagrangian perturbations and the matter bispectrum I: fourth-order model for non-linear clustering, JCAP 06 (2012) 021, [1203.4260]. 